\title{
1 Lysosomal retargeting of Myoferlin mitigates membrane stress to enable 2 pancreatic cancer growth.
}

4 Suprit Gupta ${ }^{1}$, Julian Yano ${ }^{1}$, Htet Htwe Htwe ${ }^{1}$, Hijai R. Shin ${ }^{2}$, Zeynep Cakir ${ }^{1}$, Thomas

5 Ituarte ${ }^{1}$, Kwun W. Wen ${ }^{3}$, Grace E. Kim³ ${ }^{3}$, Roberto Zoncu², David W. Dawson ${ }^{4}$, Rushika M.

6 Perera ${ }^{1,3,5}$ *

$8{ }^{1}$ Department of Anatomy, University of California, San Francisco, San Francisco, CA 94143, USA; ${ }^{2}$ Department of 9 Molecular and Cell Biology, University of California Berkeley, Berkeley, CA 94720; ${ }^{3}$ Department of Pathology,

10 University of California, San Francisco, San Francisco, CA 94143, USA; ${ }^{4}$ Department of Pathology and Laboratory

11 Medicine and Jonsson Comprehensive Cancer Center, David Geffen School of Medicine at University of California Los

12 Angeles, Los Angeles, CA 90095-1732, USA; ${ }^{5}$ Helen Diller Family Comprehensive Cancer Center, University of

13 California, San Francisco, San Francisco, CA 94158, USA.

14

15

16

17 * Corresponding author: R.M.P (rushika.perera@ucsf.edu) 


\section{Abstract}

19 Lysosomes must maintain integrity of their limiting membrane to ensure efficient fusion

20 with incoming organelles and degradation of substrates within their lumen. Pancreatic

21 cancer cells upregulate lysosomal biogenesis to enhance nutrient recycling and stress

22 resistance, but whether dedicated programs for maintaining lysosomal membrane

23 integrity facilitate pancreatic cancer growth is unknown. Using proteomic-based organelle

24 profiling, we identify the Ferlin family plasma membrane repair factor, Myoferlin, as

25 selectively and highly enriched on the membrane of pancreatic cancer lysosomes.

26 Mechanistically, lysosome localization of Myoferlin is necessary and sufficient for

27 maintenance of lysosome health and provides an early-acting protective system against

28 membrane damage that is independent from the endosomal sorting complex required for

29 transport (ESCRT)-mediated repair network. Myoferlin is upregulated in human

30 pancreatic cancer, predicts poor survival, and its ablation severely impairs lysosome

31 function and tumour growth in vivo. Thus, retargeting of plasma membrane repair factors

32 enhances pro-oncogenic activities of the lysosome. 
Lysosomes function as critical nodes for macromolecular recycling, vesicle

34 trafficking, metabolic reprogramming, and pro-growth signaling in the cell ${ }^{1-3}$. Pancreatic

35 ductal adenocarcinoma (PDA), are highly reliant on enhanced lysosome function to

36 facilitate degradation, clearance and recycling of cellular material delivered by increased

37 rates of vesicle trafficking through autophagy and macropinocytosis ${ }^{4-9}$. To cope with the

38 increased flux of substrates delivered to the lysosome for degradation, PDA cells

39 upregulate transcriptional programs for lysosome biogenesis, which are mediated by the

40 MiT/TFE factors ${ }^{6,10}$. Although enhanced MiT/TFE activity leads to a dramatic increase in

41 the number of lysosomes ${ }^{6}$, whether qualitative differences endow PDA lysosomes with

42 unique structural and functional properties to cope with higher rates of vesicle trafficking

43 and substrate clearance remains unknown.

44 An emerging aspect is the susceptibility of lysosomes to undergo membrane 45 damage due to various cellular stressors. In response to damage, several mechanisms 46 ensure proper lysosomal integrity and function by responding to damage and facilitating 47 repair or removal of dysfunctional lysosomes ${ }^{11,12}$. These pathways include repair via the 48 Endosomal Sorting Complex Required for Transport (ESCRT), a multi-subunit 49 membrane-remodeling machinery that performs membrane bending and scission away 50 from the cytoplasm ${ }^{12}$. ESCRT components were recently shown to polymerize on the 51 limiting membrane of lysosomes challenged with membrane-damaging agents, where 52 they facilitate repair of 'microtears' likely through scission and resealing of the free 53 membrane edges ${ }^{13,14}$. When ESCRT recruitment to damage sites is blocked, more 54 permanent and severe damage to lysosome function results. A second pathway facilitates 55 clearance of irreversibly damaged or dysfunctional lysosomes via a selective 56 macroautophagy process, termed lysosphagy ${ }^{11,15-17}$.

57 PDA cells are unusual in their ability to import and degrade a large variety and 58 quantity of intracellular and extracellular substrates to the lysosomal lumen in order to 59 sustain unrestrained growth in nutrient-poor microenvironments. While it is likely that this 60 enhanced substrate intake may pose unique challenges, it remains unknown whether 61 cancer-specific mechanisms for lysosomal membrane stabilization enable PDA cells to 62 cope with higher susceptibility to organelle stress. To answer this question, we conducted 63 mass spectrometry-based profiling of immuno-isolated lysosomes captured from PDA 
64 and non-PDA cells. We uncovered profound differences in the protein content and 65 composition of PDA lysosomes and identified members of the Ferlin family of membrane

66 repair factors, Myoferlin (MYOF) and Dysferlin (DYSF) as membrane proteins uniquely

67 enriched in PDA lysosome fractions, with MYOF being broadly upregulated in PDA cell

68 lines and patient samples. We find that lysosomal MYOF protects against a variety of

69 membrane stressors to sustain enhanced functionality of PDA lysosomes. Accordingly,

70 MYOF depletion in PDA cells triggers constitutive lysosomal membrane damage, leading

71 to profound defects in lysosome morphology and function and arrested PDA tumour

72 growth. Importantly, MYOF is upregulated in patient PDA specimens and high levels are

73 predictive of poor patient prognosis, thus implicating MYOF as a key regulator of

74 lysosome function and PDA tumour growth. 


\section{Results}

76 Organelle proteomics identifies the Ferlin repair factors as PDA specific lysosomal membrane proteins.

To identify novel proteins specific to PDA lysosomes, we captured intact lysosomes via affinity purification from cells stably expressing the lysosome membrane protein TMEM192 fused to mRFP and 3x HA tag (T192-mRFP-3xHA; LysoTag) (Fig. 1a; Supplementary Fig. 1a). Expression of LysoTag allows for efficient and rapid capture of intact lysosomes from cells that are amenable to mass spectrometry-based proteomics analysis $^{18,19}$. Comparative analysis of proteins present in lysosome fractions from PDA cells (PaTu8988T) versus non-PDA cells (HEK293T) identified 376 proteins with $\geq 2$-fold enrichment in PDA lysosomal elutes (Supplementary Table 1). Analysis of the biological processes and pathways associated with these enriched PDA lysosomal proteins identified pathways associated with metabolism ("fatty acid degradation", "valine, leucine, isoleucine degradation", "central carbon metabolism in cancer"), as well as cell adhesion ("focal adhesion", "ECM-receptor interaction") (Fig. 1b). Moreover, we noted that proteins associated with "vesicle-mediated trafficking" (eg. RAB22A, SNX11) and "endocytosis" (eg. CLTB, DNML1, PACSIN3, ITSN1, EZR) were significantly enriched in PDA lysosome elutes consistent with heightened rates of vesicle trafficking converging on the lysosome in PDA cells (Fig. 1b,c; Supplementary Table 2). Moreover, several autophagy related proteins were enriched in PDA lysosomes, such as LC3B, GABARAP2 and autophagy receptors (NBR1, WDFY3, SEC62, TEX264, P62), consistent with increased rates of autophagy in PDA (Fig. 1d,e). As a control, levels of LAMP1 were similar in HEK293T and PaTu8988T lysosomes (Fig. 1d,e).

Interestingly, two of the most significantly enriched proteins in PDA lysosome fractions were Myoferlin (MYOF; 30-fold enriched) and Dysferlin (DYSF; 89.6-fold enriched) (Fig. 1d,e), both of which belong to the Ferlin family of membrane repair

101 factors ${ }^{20-22}$. We first validated the PDA-specific enrichment of MYOF and DYSF via direct

102 immunoblotting of lysosomal fractions from HPDE (human pancreatic ductal epithelial 103 cells) and a panel of PDA cells expressing LysoTag. Consistent with our proteomics data, 104 PaTu8988T lysosome fractions expressed both MYOF and DYSF (Fig. 1f). Lysosomes 105 isolated from KP4 and MiaPaca cells also displayed high levels of MYOF relative to HPDE 
106 lysosomes (Fig. 1f). Moreover, lysosomal enrichment of Ferlins correlated with their

107 elevated expression in multiple PDA lines, whereas these proteins showed low or

108 undetectable expression in a panel of non-PDA cells (Fig. 1g). Of note, MYOF transcript

109 and protein levels were broadly upregulated in virtually all human PDA cell lines while

110 DYSF upregulation was restricted to 3 cell lines (Supplementary figure 1b) with

111 PaTu8988T cells having higher levels of DYSF relative to MYOF (Fig. 1g).

112 Prior studies have shown that Ferlin proteins localize predominantly to the plasma

113 membrane, where they facilitate repair of the lipid bilayer in tissues subjected to

114 heightened mechanical stress, particularly in skeletal muscle cells ${ }^{21,23,24}$. Accordingly,

115 mutations in DYSF are associated with two forms of muscular dystrophy; Limb Girdle

116 Muscular Dystrophy type 2B (LGMD2B) and Miyoshi Myopathy (MM), whereby impaired

117 membrane resealing compromises myoblast maturation, fusion and plasma membrane

118 repair $20,25,26$. Our lysosomal mass spectrometry analysis suggests that, in PDA cells,

119 Ferlin proteins may be retargeted to the lysosomal membrane. Consistent with this

120 hypothesis, transient expression of MYOF-HA in PDA cells (KP4, PaTu8902, MiaPaca

121 and Panc0203) followed by immuno-fluorescence staining confirmed a punctate

122 distribution of MYOF which colocalized with LAMP2 positive lysosomes, with little or no

123 detectable signal on the plasma membrane (Fig. 1h; Supplementary Fig. 1c). In

124 contrast, MYOF-HA localized to the plasma membrane or showed a diffuse cytoplasmic

125 localization in non-PDA cells with no visible overlap with LAMP2-positive structures (Fig.

126 1h; Supplementary Fig. 1d). To further confirm the differential subcellular targeting of

127 MYOF in PDA versus non-PDA cells we performed biotin labeling of surface proteins in

128 KP4 cells and HEK293T cells transiently expressing MYOF-HA followed by streptavidin-

129 mediated immunoprecipitation. Consistent with the immunofluorescence results, we find

130 that endogenous MYOF is not biotinylated in KP4 cells and therefore absent from the

131 plasma membrane (Fig. 1i). In contrast MYOF transiently expressed in HEK293T cells

132 was efficiently biotinylated, consistent with its plasma membrane localization in these

133 cells (Fig. 1h,i). Thus, Myoferlin is both upregulated and show substantial retargeting to

134 the lysosome in PDA cells. Given the preferential upregulation of MYOF in PDA cells

135 relative to HPDE, we focused our functional analysis on this Ferlin family member in

136 subsequent experiments. 
To establish that MYOF resides on the outer (cytoplasm-facing) leaflet of the lysosomal membrane rather than being trafficked to the lysosomal lumen for degradation, we first treated cells with the lysosome V-ATPase inhibitor Bafilomycin A1 (BafA1) to

140 inhibit lysosomal degradation. BafA1 treatment led to a progressive accumulation of 141 known cargo proteins such as the autophagy receptor p62 (Supplementary Fig. 1e). In 142 contrast MYOF did not show an increase in levels upon BafA1 treatment suggesting that 143 it is unlikely to be a cargo protein brought to the lysosome for degradation.

144 To further prove that MYOF is anchored to the cytoplasmic face of the lysosomal membrane, we treated affinity-captured PDA lysosomes with increasing concentrations

146 of proteinase $\mathrm{K}$, with the expectation that cytoplasmic facing proteins would be sensitive 147 to proteinase $\mathrm{K}$ digestion while luminal proteins would be protected. As expected, luminal 148 proteins (LC3, Cathepsin C) and luminal facing proteins (LAMP2) were protected from 149 proteinase $\mathrm{K}$ digestion, whereas membrane proteins exposed to the cytoplasm such as $150 \quad$ NPC1 were progressively digested with increasing concentrations of proteinase K (Fig. 151 1j). MYOF showed rapid digestion by proteinase $K$, confirming that it is a membrane 152 protein present on the outer surface of the lysosome (Fig. 1j). Taken together these data 153 indicate that MYOF is topologically anchored via its C-terminal transmembrane domain 154 to the outer lysosomal membrane, with its $\mathrm{N}$-terminus extending into the cytoplasm.

\section{PDA lysosomes display enhanced protection against membrane damage.}

Given a known function for Ferlin proteins in maintenance and repair of membrane, we sought to determine whether lysosomal localization of MYOF might confer enhanced 159 protection against membrane damage. To do so, we compared the response of PDA 160 lysosomes versus non-PDA lysosomes under conditions that mimic various forms of 161 lysosomal stress. We first employed acute, chemically-induced lysosomal membrane 162 damage by treating cells with LLOMe (L-Leucyl-L-leucine O-methyl ester), a well163 established lysosomotropic agent commonly used to rupture endolysosomal 164 membranes ${ }^{13,14,27}$. LLOMe rapidly and efficiently accumulates within the lumen of acidic 165 organelles where it is converted by the lysosomal protease Cathepsin C into a polymer 166 capable of causing small ruptures in the membrane ${ }^{27}$. To test the response of lysosomes 167 to the LLOMe challenge, cells were preloaded with Lysotracker red dye, which 
168 accumulates within intact lysosomes, and the rate of dye leakage induced by LLOMe was

169 measured over time. While lysosomes in non-PDA cells (HPDE, U20S and HEK293T)

170 lost virtually all lysotracker red staining within 15min of LLOMe treatment (Fig. 2a;

171 Supplementary Fig. 2a), consistent with progressive lysosomal membrane rupture, PDA

172 lysosomes retained the Lysotracker dye for longer time periods (Fig. 2a,b). This effect

173 was not due to reduced Cathepsin C-dependent activation of LLOMe in PDA lysosomes,

174 as Cathepsin C levels were similar, if not higher in PDA cells relative to HPDE cells

175 (Supplementary Fig. 2b). Prior studies have also established that particulate material

176 such as crystals of silica, alum and uric acid are capable of damaging vesicles and

177 lysosomal membrane ${ }^{14}$. Similarly, changes in osmolarity have also been shown to cause

178 changes in endolysosome membrane tension and permeability ${ }^{28}$. Therefore, we

179 measured the response of PDA and non-PDA cells to these additional forms of

180 endolysosome membrane damage. Consistent with our observations with LLOMe, we

181 find that PDA lysosomes are more resistant to silica-induced and hypertonic sucrose-

182 induced membrane damage as measured by reduced loss of Lysotracker red dye signal

183 (Supplementary Fig. 2c-f). Collectively, these data suggest that, relative to non-PDA

184 counterparts, PDA lysosomes are capable of withstanding diverse membrane perturbing

185 agents, relative to non-PDA lysosomes.

186 Along with its degradative functions, the lysosome serves as the platform for

187 nutrient signaling via the mechanistic target of rapamycin complex 1 (mTORC1), which

188 must localize to the lysosome surface ${ }^{1,19}$ and requires an intact lysosomal membrane in

189 order to be activated ${ }^{16}$. Treatment of HPDE cells with LLOMe over a $1 \mathrm{~h}$ time course led

190 to a rapid decrease in mTORC1 signaling as measured by phosphorylation of

191 downstream targets p70S6K and 4EBP1 (Fig. 2c). In contrast, PDA cells retained

192 mTORC1 signaling activity throughout the 1h LLOMe treatment duration (Fig. 2c;

193 Supplementary Fig. 2g). Similarly, a 4-fold lower dose of LLOMe was sufficient to

194 suppress mTORC1 signaling in HPDE cells, while PDA cells retained mTORC1 signaling

195 even at the highest dose of LLOMe tested (Supplementary Fig. 2h).

196 Recent studies showed that lysosomal membrane disruption is accompanied by

197 rapid recruitment of ESCRT machinery that facilitates membrane repair ${ }^{13,14}$. ESCRT

198 proteins are organized into sub-complexes; ESCRT III proteins (CHMPs 1-7 and IST1) 
199 are responsible for mediating membrane constriction and fission whereas ALIX (ESCRT

200 II) and TSG101 (ESCRT I) facilitate recruitment of ESCRT III components to the site of 201 damage ${ }^{13,14}$. Immunofluorescence staining following a time course of LLOMe, Silica or

202 hypertonic sucrose treatment showed that HPDE cells rapidly recruit ALIX to lysosomes 203 post treatment (Supplementary Fig. 3a-c). In contrast, identically treated KP4 cells 204 showed a significant delay in ALIX recruitment (Supplementary Fig. 3a-c). Similarly, 205 LLOMe treatment lead to rapid requirement of ESCRT III proteins CHMP3 and CHMP1A 206 as early as 1 min post treatment in HPDE cells and was significantly delayed in PDA cells 207 (Fig. 2d,e; Supplementary Fig. 3d,e). Additional markers of lysosomal membrane 208 damage include the Galectins - cytoplasmic proteins that recognize and bind to 209 inappropriately exposed glycans on the luminal side of lysosomal membrane 210 proteins ${ }^{15,17,29,30}$. Within 15 min of LLOMe treatment 45\% of LAMP2 positive lysosomes in 211 HPDE cells recruited Galectin 3 (GAL3) (Fig. 2f,g). In contrast, only 8\% of lysosomes in 212 PDA cells recruited GAL3, which showed predominantly diffuse staining in PDA cells even 213 after 30min of LLOMe treatment (Fig. 2f,g). To further confirm differential recruitment of 214 GAL3 to lysosomes in response to LLOMe in KP4 and HPDE cells we captured intact 215 lysosomes from LysoTag expressing cells following treatment with LLOMe for 10 minutes. 216 Lysosomes purified from LLOMe treated HPDE cells contained higher levels of GAL3 as 217 measured by immunoblot, compared to lysosomes purified from identically treated KP4 218 cells (Supplementary Fig. 3f). These findings support the notion that PDA lysosomes 219 display enhanced protection against acute membrane damage compared to their non220 PDA counterparts. Myoferlin is required to maintain lysosome function in PDA cells.

The unique protein composition of PDA lysosomes may help maintain their 224 membrane integrity and function in response to stress. Based on the known membrane 225 repair functions of the Ferlins, we next tested whether lysosomal localization of MYOF 226 could explain the increased stress resistance observed of PDA lysosomes. Suppression 227 of MYOF expression via shRNA mediated knockdown or CRISPR mediated knockout in 228 PDA cell lines led to lysosomes with aberrant morphology and increased diameter (Fig. 229 3a; Supplementary Fig. 4a-d) - a phenotype commonly associated with lysosome 
230 dysfunction ${ }^{31,32}$. Electron microscopy analysis of PDA cells following knockdown of MYOF 231 confirmed an abundance of enlarged lysosomes which lacked intraluminal content (Fig.

232 3b). This phenotype is distinct from general inhibition of lysosome digestion following 233 treatment with lysosomotropic agents (Chloroquine), V-ATPase inhibitors (BafA1) ${ }^{33}$ or 234 knockdown of MiT/TFE transcription factors ${ }^{6}$, which commonly result in distended 235 lysosomes filled with undigested, electron-dense content. Consistent with this idea, 236 MYOF deficient lysosomes were unable to accumulate intraluminal Lysotracker dye 237 (Supplementary Fig. 4e). Importantly, MYOF loss triggered a spontaneous lysosomal 238 membrane stress response, associated with constitutive localization of ESCRT proteins 239 ALIX, CHMP3 and CHMP1A to LAMP1 positive lysosomes (Fig. 3c,d; Supplementary 240 Fig. 4f). Together, the alteration in lysosome morphology, lack of Lysotracker dye 241 retention and constitutive recruitment of ESCRT proteins suggests that MYOF serves to 242 protect against lysosome dysfunction in PDA cells. Accordingly, knockdown of MYOF 243 caused a marked accumulation of lipidated LC3B by immunoblotting (Fig. 3e) and 244 increased LC3B-positive puncta by immunofluorescence (Fig. 3f; Supplementary Fig. $245 \mathbf{4 g}$ ) as well as delayed clearance of macropinocytosed serum albumin, indicating 246 defective lysosomal proteolysis (Supplementary Fig. 4h). Moreover, loss of MYOF also 247 led to reduced baseline mTORC1 signaling as measured by phosphorylation of p70S6K 248 and 4EBP1, which was suppressed further following treatment with LLOMe 249 (Supplementary Fig. 4i). These data support a novel role for MYOF in maintenance of 250 lysosome function in PDA cells.

251 PDA cells are characterized by elevated autophagy and macropinocytosis, which 252 delivers intracellular and extracellular cargo, respectively, to the lysosome for 253 degradation ${ }^{4,7,8}$. This increased cargo trafficking may create a unique dependency on 254 MYOF to maintain membrane stability and efficient lysosome function. Therefore, 255 reducing the incoming flux of membrane trafficking should decrease the dependency of 256 PDA cells on MYOF. To explore this idea, we tested whether blocking autophagosome 257 formation and trafficking via knockdown of ATG3 or ATG7 might prevent the lysosomal 258 membrane stress observed in MYOF KO cells. Knockdown of ATG3 or ATG7 in MYOF 259 KO cells led to reduced autophagy as evidenced by accumulation of p62 and unlipidated 260 LC3B-I (Fig. 3g). Strikingly, autophagy suppression resolved the lysosome stress 
261 response induced by MYOF loss, as indicated by reduced ALIX recruitment to lysosomes

262 in MYOF KO cells (Fig. 3h,i). These data suggest that the increased vesicle trafficking

263 characteristic of PDA cells imparts heightened stress on the lysosome in order to fuse,

264 process and clear incoming cargo; this stress is counteracted by increased expression

265 and lysosomal targeting of MYOF.

Inducible lysosome targeting of MYOF is sufficient to protect against acute membrane damage.

To gain insights into the mechanism of MYOF-mediated lysosomal stabilization, we tested whether inducible targeting of MYOF to the lysosomal membrane in non-PDA cells would be sufficient to protect against LLOMe-mediated damage. To do so, we used

272 a heterodimerization system (FKBP-FRB) to inducibly recruit a variant of MYOF lacking 273 its C-terminal transmembrane domain (MYOF $\triangle$ TM) (Fig. 4a), to the lysosome membrane 274 following addition of a rapamycin-derived chemical dimerizer AP21967 (AP) ${ }^{34,35}$ (Fig. 4b).

275 U2OS cells were first engineered to stably express the FKBP module conjugated to the 276 C-terminus of the lysosomal membrane protein TMEM192 (T192-Flag-FKBP) ${ }^{34,35}$, which 277 we confirmed localizes to LAMP2 positive lysosomes (Fig. 4c). These cells were then 278 transiently transfected with MYOF $\Delta$ TM conjugated to FRB* (referred to hereafter as 279 MYOF-FRB*$^{*}$. In the absence of AP, MYOF-FRB* showed a diffuse cytoplasmic 280 localization (Fig. 4d; top) in T192-Flag-FKBP expressing U2OS cells. Upon treatment 281 with AP, MYOF-FRB* was massively recruited to LAMP2 positive lysosomes (Fig. 4d; 282 bottom). Without AP treatment, addition of LLOMe to U2OS cells led to recruitment of 283 ALIX (Fig. 4e) and GAL3 (Supplementary Fig. 5a) to lysosomes as expected. However, 284 following addition of $\mathrm{AP}$ and the resulting recruitment of MYOF-FRB* to lysosomes, 285 recruitment of ALIX and GAL3 to lysosomes was significantly reduced in response to 286 LLOMe (Fig. 4e; Supplementary Fig. 5a). These data suggest that targeting of MYOF 287 to the membrane of non-PDA lysosomes is sufficient to confer protection against 288 chemically induced membrane damage.

289 The first three C2 domains of MYOF have been shown to bind membrane lipids, 290 alter their distribution ${ }^{36,37}$ and recruit accessory proteins ${ }^{38}$ and are therefore thought to be 291 key mediators of the membrane resealing functions of MYOF and DYSF. We therefore 
292 tested whether a variant of MYOF lacking C2A, C2B and C2C domains (MYOF $\triangle$ C2)

293 would lead to loss of membrane protection following LLOMe-mediated damage in non-

294 PDA cells. Unlike MYOF-FRB* ${ }^{*}$ AP mediated recruitment of the MYOF $\triangle C 2-F R B^{*}$ mutant

295 to T192-Flag-FKBP positive lysosomes was unable to prevent LLOMe-induced damage,

296 as shown by unchanged ALIX and GAL3 recruitment irrespective of the AP dimerizer

297 (Fig. 4f; Supplementary Fig. 5b). These data establish the N-terminal C2 domains of

298 MYOF as mediating its protective function at the lysosome.

Loss of MYOF leads to impaired in vivo tumour growth.

Given the established role for lysosomes as mediators of cellular adaptation and growth in PDA, we next determined the impact of MYOF loss on PDA growth in vitro and in vivo. Similar to human PDA cell lines (Fig. 1g), tumours isolated from a genetically engineered mouse model of PDA (p48-Cre;LSL-Kras ${ }^{\mathrm{G} 12 \mathrm{D}} ; \operatorname{Trp53}^{\mathrm{L} /+}$; referred to here as $\mathrm{KPC})^{39}$ displayed levels of Myof that were higher than normal pancreas and liver, and comparable to mouse C2C12 muscle cells (Fig. 5a). Dysf also showed higher levels in KPC tumours relative to normal pancreas (Fig. 5a). Importantly, Myof expression in KPC tumours was restricted to CK19 positive tumour epithelia and did not show significant overlap with $\alpha$-SMA positive stromal cells (Fig. 5b). CRISPR mediated knockout of Myof in murine KPC cells ${ }^{40}$ led to accumulation of LC3B-II, reduced accumulation of

311 Lysotracker dye and reduced in vivo growth following transplantation in syngeneic hosts

312 (Supplementary Fig. 6a-d). Similarly, shRNA mediated knockdown or CRISPR mediated 313 knockout of MYOF in KP4 cells significantly reduced colony forming ability (Fig. 5c) and 314 in vivo tumour growth (Fig. 5d,e). Resected tumours also showed a decrease in 315 proliferation as measured by Ki67 staining (Fig. 5f). Together these data strongly support 316 a critical role for MYOF as a novel lysosomal membrane protein essential for maintenance 317 of organelle function during tumour growth in vivo.

\section{MYOF levels dictate worse prognosis in PDA patients.}

Consistent with high expression levels of MYOF in human PDA cell lines and

321 murine KPC tumours, analysis of several publicly available patient PDA datasets showed 322 that MYOF transcript levels were significantly higher in human PDA tumours relative to 
323 normal pancreas or adjacent non-neoplastic tissue (Fig. 6a). In addition, semi324 quantitative immunohistochemical analysis revealed significantly increased MYOF 325 protein expression in patient-matched neoplastic versus non-neoplastic epithelium ( $\mathrm{n}=$ $\left.326102, P<2.2 \times 10^{-25}\right)$ with average histoscores of $3.95 \pm 2.3$ versus $0.57 \pm 0.82$, 327 respectively. Further dichotomization (histoscores $>5$ versus $\leq 5$ ) revealed high MYOF in $32823 \%$ of PDA versus $0 \%$ of adjacent normal pancreas, $(\mathrm{n}=102 ; P<0.0001)$ (Fig. 6b,c). 329 No significant associations were noted between high MYOF PDA and clinicopathologic 330 parameters except for a significant correlation with female gender $(P=0.008$;

331 Supplementary Table 3). Notably, high MYOF PDA was associated with worse overall 332 survival in univariate analysis (hazard ratio $=2.03 ; 95 \% \mathrm{Cl}=1.29-3.19$; Supplementary 333 Table 4a) with a median overall survival of 19.4 months compared to 32 months for 334 patients with low MYOF PDA (log rank $P=0.002$, Fig. 6d). High MYOF expression was 335 also an independent predicator of worse overall survival in multivariate Cox regression 336 analysis (Supplementary Table 4b). Moreover, a significant correlation between high 337 MYOF expression and worse overall survival was confirmed in an independent PDA 338 patient cohort from The Cancer Genome Atlas (Fig. 6e). Together, analyses from these 339 clinical datasets support a role for MYOF and enhanced lysosome function as predictors 340 of shortened survival in PDA patients. 


\section{Discussion}

Our findings demonstrate that PDA lysosomes are intrinsically more capable of

344 withstanding membrane stress than non-cancer lysosomes. Retargeting of the Ferlin

345 family of plasma membrane repair factors to the lysosomal membrane is critical for this

346 ability and helps to maintain the structural integrity and pro-tumourigenic activities of this

347 organelle. Lysosomal targeting of the Ferlins appears to be an adaptive mechanism in

348 response to the increased degradative burden placed on PDA lysosomes by elevated

349 influx of autophagic and macropinocytic vesicle trafficking (Fig. 6f). Consistent with this

350 model, inhibition of autophagy relieves the lysosomal membrane stress that accumulated

351 following MYOF loss.

352 The nature of the membrane stress that PDA lysosomes undergo may derive from

353 the specific cargo being degraded, such as large protein aggregates of intracellular or

354 extracellular origin, oxidative damage as well as alterations in membrane composition 355 due to continuous fusion with autophagy-derived and plasma membrane-derived 356 vesicles. Ferlins may counteract this stress through several mechanisms, including 357 formation of oligomers that structurally stabilize the lipid bilayer, thus preventing its 358 rupture, as well as promoting fusion with other vesicles that act as membrane donors to 359 reseal damage that has already occurred. Both mechanisms have been proposed to 360 underlie Ferlin-mediated repair of the plasma membrane of skeletal muscle cells and may 361 operate at the lysosomal membrane in PDA cells as well $21,23,24,37$.

362 Our findings also suggest that MYOF either replaces or delays activation of a major 363 membrane repair system mediated by the ESCRT complex. ESCRT is thought to repair 364 membrane 'microtears' through scission of the free membrane edge into the lumen and 365 resealing of the 'neck' driven by subunit polymerization ${ }^{21,23,24,37}$. In turn, this resealing 366 activity is critical to prevent exposure and leakage of luminal lysosomal proteins and 367 protein domains. In the simplest model, the membrane-stabilizing action of MYOF may 368 inhibit or delay ESCRT recruitment by preventing the formation of exposed microtears. 369 Consistent with this idea, loss of MYOF in PDA cells triggers spontaneous ESCRT 370 recruitment to the lysosome. More direct mechanisms of Ferlin mediated ESCRT 371 regulation may also occur, such as competition for a common binding partner. 
372 Alternatively, Ferlin proteins may represent a new protective mechanism in cells highly

373 reliant on the lysosome for growth. Given that ESCRT proteins have diverse functions at

374 multiple cellular locations ${ }^{12}$, a dedicated lysosomal program may confer more efficient

375 monitoring and maintenance of lysosomal health.

376 Accordingly, loss of MYOF leads to severe lysosomal dysfunction and significantly

377 impairs PDA tumour growth in vivo, establishing a critical role for this protein in promoting

378 the pro-oncogenic functions of the lysosome in cancer. PDA cells rely on lysosomes as

379 an important source of metabolites ${ }^{2}$ and the lysosome regulates levels of essential 380 micronutrients including iron ${ }^{41,42}$ and calcium $^{43}$ that can be exchanged with other 381 organelles in the cell including the $\mathrm{ER}^{44,45}$ and mitochondria ${ }^{41,42}$. Thus, lysosome 382 dysfunction following MYOF loss could indirectly impact additional organelles and cellular 383 metabolic processes. In line with this hypothesis, prior studies ${ }^{46,47}$ showed that MYOF 384 loss in cancer cells caused respiratory defects in mitochondria, an organelle where MYOF 385 is not found. Finally, high MYOF expression distinguishes a cohort of patient PDA 386 tumours that predict worse overall survival. Several other cancers also show elevated 387 MYOF expression ${ }^{47-50}$ however a role for MYOF in regulation of lysosome function in 388 these cancers remains unknown.

389 Our findings highlight unique features of cancer lysosomes and identify a 390 dedicated protective mechanism essential for maintenance of lysosomal health. Blocking 391 this protective function of MYOF may pave the way for new lysosome-centered strategies 392 for inhibiting PDA and other cancers. 


\section{Acknowledgements}

394 We thank all the members of the Perera lab and Debnath Lab for helpful discussions. R.M.P is 395 the Nadia's Gift Foundation Innovator of the Damon Runyon Cancer Research Foundation (DRR-

396 46-17) and is additionally supported by an NIH Director's New Innovator Award (1DP2CA216364), 397 the Pancreatic Cancer Action Network Career Development Award, and the Hirshberg Foundation

398 for Pancreatic Cancer. H.R.S. is supported by an AACR-Amgen fellowship in 399 Clinical/Translational Cancer Research. R.Z is supported by grants from the NIH (R01GM127763, 400 R01GM130995), a Damon Runyon-Rachleff Innovator Award, Edward Mallinckrodt, Jr. 401 Foundation Grant. D.W.D. receives support from the Hirshberg Foundation for Pancreatic Cancer 402 Research. We thank Reena Zalpuri at the University of California Berkeley Electron Microscope 403 Laboratory for advice and assistance in electron microscopy sample preparation and data 404 collection.

\section{Author contributions}

407 S.G. performed the majority of experiments and drafted the manuscript. J.Y. developed the FKBP408 FRB assay, conducted surface biotinylation experiments, molecular cloning and data analysis. 409 H.H.H. assisted with mouse experiments and immuno-histochemistry. H.R.S. performed the 410 electron microscopy. Z.C. performed the proteinase $\mathrm{K}$ protection assay T.I conducted data and 411 pathway analysis. K.W.W and G.K provided pathology analysis of patient samples. R.Z. provided 412 intellectual feedback, reagents and supervised H.R.S. D.W.D provided the PDA TMA and 413 conducted independent pathology analysis and statistical testing. R.M.P. conceived the project, 414 supervised the research, wrote and edited the manuscript.

\section{Competing interests}

417 The other authors declare no competing interests. 


\section{References}

4201 Lawrence, R. E. \& Zoncu, R. The lysosome as a cellular centre for signalling, metabolism and quality control. Nat Cell Biol 21, 133-142, doi:10.1038/s41556-018-0244-7 (2019).

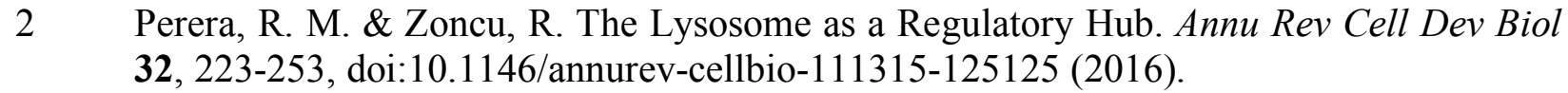

3 Ballabio, A. \& Bonifacino, J. S. Lysosomes as dynamic regulators of cell and organismal homeostasis. Nat Rev Mol Cell Biol 21, 101-118, doi:10.1038/s41580-019-0185-4 (2020).

4 Commisso, C. et al. Macropinocytosis of protein is an amino acid supply route in Rastransformed cells. Nature 497, 633-637, doi:10.1038/nature12138 (2013).

5 Kamphorst, J. J. et al. Human pancreatic cancer tumors are nutrient poor and tumor cells actively scavenge extracellular protein. Cancer Res 75, 544-553, doi:10.1158/00085472.CAN-14-2211 (2015).

6 Perera, R. M. et al. Transcriptional control of autophagy-lysosome function drives pancreatic cancer metabolism. Nature 524, 361-365, doi:10.1038/nature14587 (2015).

7 Yang, A. et al. Autophagy is critical for pancreatic tumor growth and progression in tumors with p53 alterations. Cancer Discov 4, 905-913, doi:10.1158/2159-8290.CD-14-0362 (2014).

Yang, S. et al. Pancreatic cancers require autophagy for tumor growth. Genes Dev 25, 717729, doi:10.1101/gad.2016111 (2011). Yamamoto, K. et al. Autophagy promotes immune evasion of pancreatic cancer by

11 Papadopoulos, C., Kravic, B. \& Meyer, H. Repair or Lysophagy: Dealing with Damaged Lysosomes. J Mol Biol 432, 231-239, doi:10.1016/j.jmb.2019.08.010 (2020).

12 Vietri, M., Radulovic, M. \& Stenmark, H. The many functions of ESCRTs. Nat Rev Mol Cell Biol 21, 25-42, doi:10.1038/s41580-019-0177-4 (2020).

13 Radulovic, M. et al. ESCRT-mediated lysosome repair precedes lysophagy and promotes cell survival. EMBO J 37, doi:10.15252/embj.201899753 (2018).

14 Skowyra, M. L., Schlesinger, P. H., Naismith, T. V. \& Hanson, P. I. Triggered recruitment of ESCRT machinery promotes endolysosomal repair. Science 360, doi:10.1126/science.aar5078 (2018).

15 Hung, Y. H., Chen, L. M., Yang, J. Y. \& Yang, W. Y. Spatiotemporally controlled induction of autophagy-mediated lysosome turnover. Nat Commun 4, 2111, doi:10.1038/ncomms3111 (2013).

Jia, J. et al. Galectins Control mTOR in Response to Endomembrane Damage. Mol Cell biogenesis and kidney injury. EMBO J 32, 2336-2347, doi:10.1038/emboj.2013.171 (2013). dependent regulation of amino acid efflux from lysosomes. Science 358, 807-813, doi:10.1126/science.aan6298 (2017). 
46319 Zoncu, R. et al. mTORC1 senses lysosomal amino acids through an inside-out mechanism

464 that requires the vacuolar $\mathrm{H}(+)$-ATPase. Science 334, 678-683, doi:10.1126/science.1207056 (2011).

46620 Bansal, D. \& Campbell, K. P. Dysferlin and the plasma membrane repair in muscular dystrophy. Trends Cell Biol 14, 206-213, doi:10.1016/j.tcb.2004.03.001 (2004).

21 Bansal, D. et al. Defective membrane repair in dysferlin-deficient muscular dystrophy. Nature 423, 168-172, doi:10.1038/nature01573 (2003).

22 Davis, D. B., Delmonte, A. J., Ly, C. T. \& McNally, E. M. Myoferlin, a candidate gene and potential modifier of muscular dystrophy. Hum Mol Genet 9, 217-226, doi:10.1093/hmg/9.2.217 (2000).

23 Doherty, K. R. et al. Normal myoblast fusion requires myoferlin. Development 132, 55655575, doi:10.1242/dev.02155 (2005).

24 Lek, A., Evesson, F. J., Sutton, R. B., North, K. N. \& Cooper, S. T. Ferlins: regulators of vesicle fusion for auditory neurotransmission, receptor trafficking and membrane repair. Traffic 13, 185-194, doi:10.1111/j.1600-0854.2011.01267.x (2012).

25 Bashir, R. et al. A gene related to Caenorhabditis elegans spermatogenesis factor fer-1 is mutated in limb-girdle muscular dystrophy type 2B. Nat Genet 20, 37-42, doi:10.1038/1689 (1998).

26 Liu, J. et al. Dysferlin, a novel skeletal muscle gene, is mutated in Miyoshi myopathy and limb girdle muscular dystrophy. Nat Genet 20, 31-36, doi:10.1038/1682 (1998).

27 Repnik, U. et al. L-leucyl-L-leucine methyl ester does not release cysteine cathepsins to the cytosol but inactivates them in transiently permeabilized lysosomes. J Cell Sci 130, 3124-3140, doi:10.1242/jcs.204529 (2017).

28 Mercier, V. et al. Endosomal membrane tension regulates ESCRT-III-dependent intralumenal vesicle formation. Nat Cell Biol 22, 947-959, doi:10.1038/s41556-020-0546-4 (2020).

29 Chauhan, S. et al. TRIMs and Galectins Globally Cooperate and TRIM16 and Galectin-3 Co-direct Autophagy in Endomembrane Damage Homeostasis. Dev Cell 39, 13-27, doi:10.1016/j.devcel.2016.08.003 (2016).

30 Thurston, T. L., Wandel, M. P., von Muhlinen, N., Foeglein, A. \& Randow, F. Galectin 8 targets damaged vesicles for autophagy to defend cells against bacterial invasion. Nature 482, 414-418, doi:10.1038/nature10744 (2012).

31 Kilpatrick, B. S., Eden, E. R., Hockey, L. N., Futter, C. E. \& Patel, S. Methods for monitoring lysosomal morphology. Methods Cell Biol 126, 1-19, doi:10.1016/bs.mcb.2014.10.018 (2015).

49832 Platt, F. M., Boland, B. \& van der Spoel, A. C. The cell biology of disease: lysosomal

33 Mauthe, M. et al. Chloroquine inhibits autophagic flux by decreasing autophagosome-

34 Lawrence, R. E. et al. A nutrient-induced affinity switch controls mTORC1 activation by its Rag GTPase-Ragulator lysosomal scaffold. Nat Cell Biol 20, 1052-1063, doi:10.1038/s41556-018-0148-6 (2018). protein translocation using nontoxic ligands identified by a mammalian three-hybrid 
36 Davis, D. B., Doherty, K. R., Delmonte, A. J. \& McNally, E. M. Calcium-sensitive phospholipid binding properties of normal and mutant ferlin C2 domains. J Biol Chem 277, 22883-22888, doi:10.1074/jbc.M201858200 (2002).

37 Marty, N. J., Holman, C. L., Abdullah, N. \& Johnson, C. P. The C2 domains of otoferlin, dysferlin, and myoferlin alter the packing of lipid bilayers. Biochemistry 52, 5585-5592, doi:10.1021/bi400432f(2013).

39 Lee, J. J. et al. Stromal response to Hedgehog signaling restrains pancreatic cancer progression. Proc Natl Acad Sci U S A 111, E3091-3100, doi:10.1073/pnas.1411679111 (2014).

40 Hingorani, S. R. et al. Preinvasive and invasive ductal pancreatic cancer and its early detection in the mouse. Cancer Cell 4, 437-450, doi:10.1016/s1535-6108(03)00309-X (2003).

41 Weber, R. A. et al. Maintaining Iron Homeostasis Is the Key Role of Lysosomal Acidity for Cell Proliferation. Mol Cell 77, 645-655 e647, doi:10.1016/j.molcel.2020.01.003 (2020).

527

42 Yambire, K. F. et al. Impaired lysosomal acidification triggers iron deficiency and inflammation in vivo. Elife 8, doi:10.7554/eLife.51031 (2019).

$43 \mathrm{Xu}, \mathrm{H} . \quad \&$ Ren, D. Lysosomal physiology. Annu Rev Physiol 77, 57-80, doi:10.1146/annurev-physiol-021014-071649 (2015). Dong, R. et al. Endosome-ER Contacts Control Actin Nucleation and Retromer Function through VAP-Dependent Regulation of PI4P. Cell 166, 408-423, doi:10.1016/j.cell.2016.06.037 (2016). (2020).

50 Blomme, A. et al. Myoferlin regulates cellular lipid metabolism and promotes metastases aberrant growth signalling in Niemann-Pick type C. Nat Cell Biol 21, 1206-1218, doi:10.1038/s41556-019-0391-5 (2019). ductal adenocarcinoma, and affects tumor aggressiveness. Oncogene 37, 4398-4412, doi:10.1038/s41388-018-0287-z (2018).

47 Rademaker, G. et al. Human colon cancer cells highly express myoferlin to maintain a fit mitochondrial network and escape p53-driven apoptosis. Oncogenesis 8, 21, Turtoi, A. et al. Myoferlin is a key regulator of EGFR activity in breast cancer. Cancer Res 73, 5438-5448, doi:10.1158/0008-5472.CAN-13-1142 (2013). in triple-negative breast cancer. Oncogene 36, 2116-2130, doi:10.1038/onc.2016.369 (2017). 
Fig. 1 | Organelle proteomics identifies the Ferlin repair factors as PDA specific lysosome associated membrane proteins.
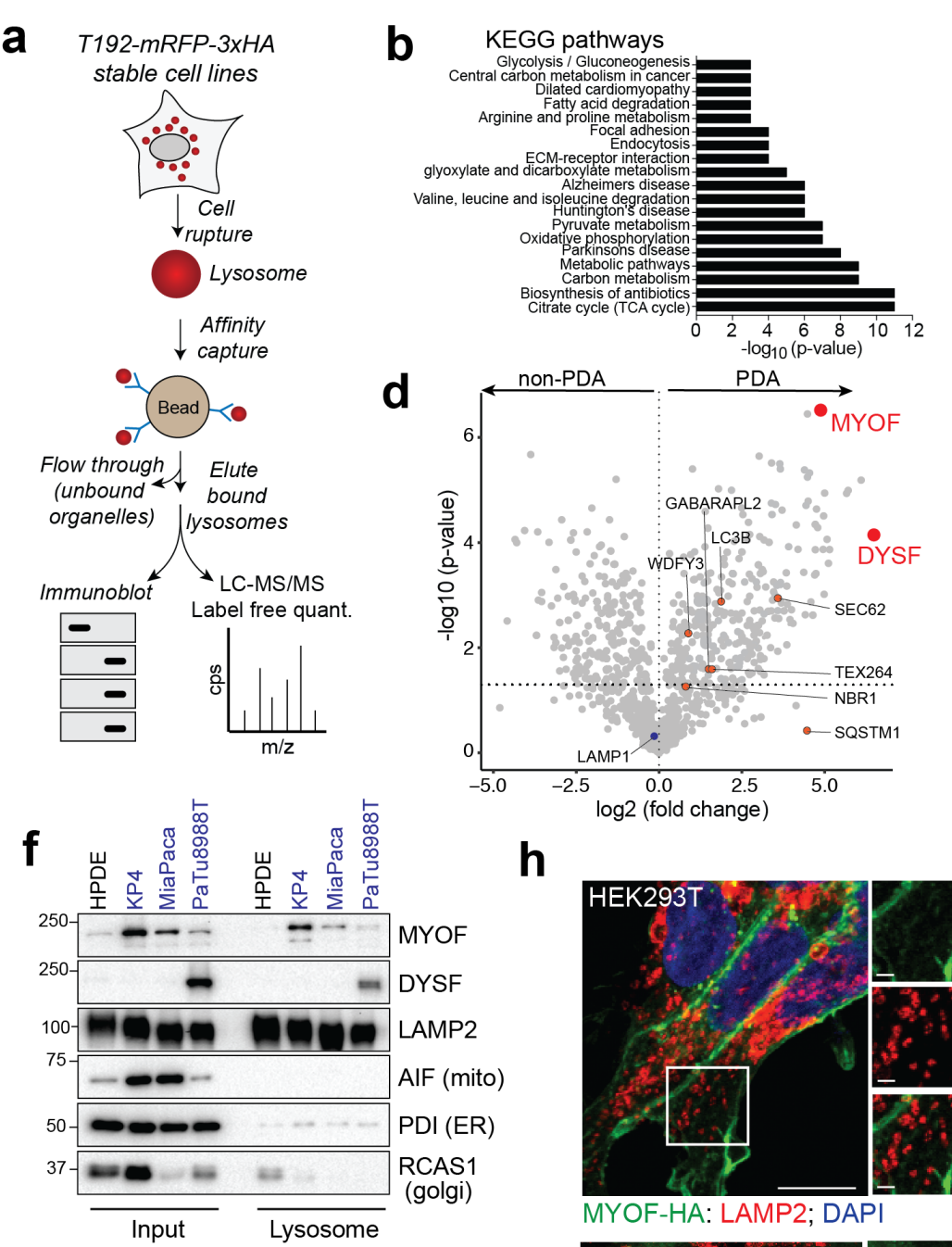

9

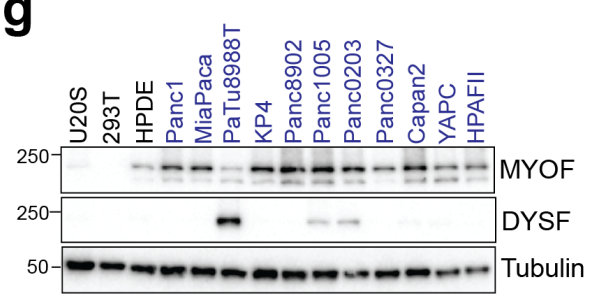

h

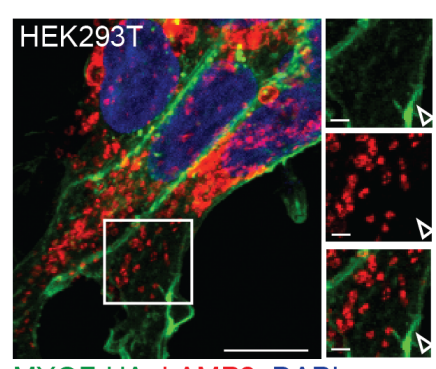

MYOF-HA: LAMP2; DAPI

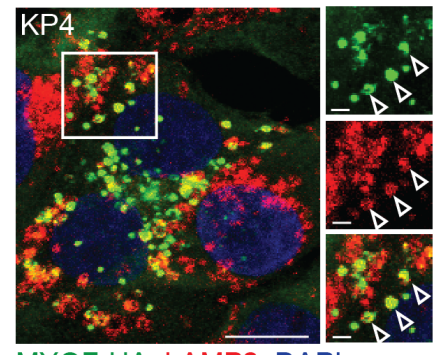

MYOF-HA: LAMP2; DAPI associated membrane proteins.
C Vesicle-mediated trafficking (GO:0016192)

O Endocytosis (GO:0006897)

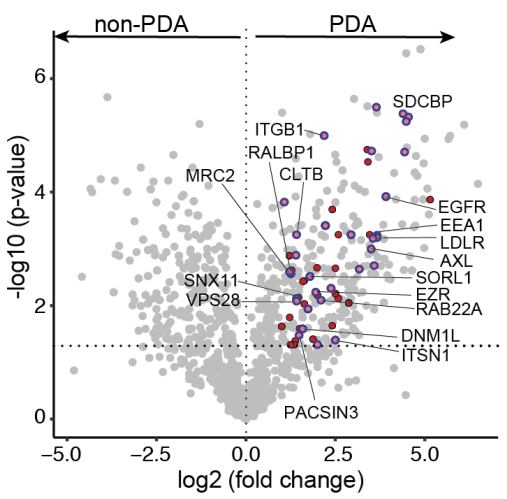

e

\begin{tabular}{|c|c|c|}
\hline Identified & \multicolumn{2}{|c|}{ Avg. peptide counts } \\
\cline { 2 - 3 } protein & normal & PDA \\
\hline MYOF & 3.6 & 108 \\
DYSF & 1.0 & 89.6 \\
LC3B & 1.0 & 3.6 \\
GABARAPL2 & 2.0 & 5.6 \\
SEC62 & 0.6 & 8.0 \\
SQSTM1 & 6.6 & 117.3 \\
LAMP1 & 69.6 & 63.0 \\
\hline
\end{tabular}

i

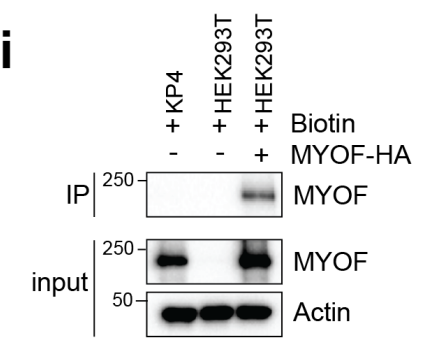

j

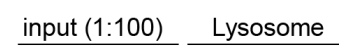

Proteinase $\mathrm{K}$

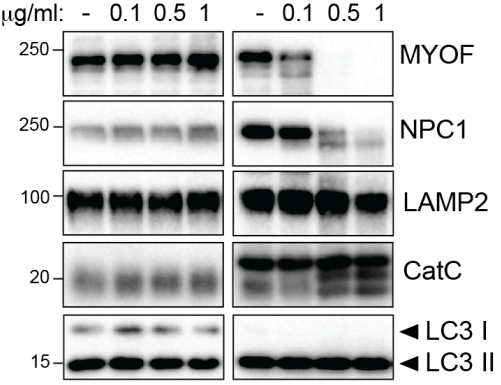

Fig. 1 | Organelle proteomics identifies the Ferlin repair factors as PDA specific lysosome

a. Schematic showing lysosome purification using affinity-based capture from cells stably expressing T192-mRFP-3xHA. b. KEGG pathway analysis of $\geq 2$-fold enriched PDA lysosome associated proteins. c. Volcano plot of lysosome proteomics data from non-PDA (HEK293T) and 
560 PDA (PaTu8988T) cells. Data are plotted as log2 fold change (PDA/non-PDA) versus the -log10

561 of the p-value. $\geq 2$-fold enriched proteins associated with "vesicle mediated trafficking" are

562 indicated in dark red and overlapping proteins associated with "endocytosis" are indicated in

563 pink/blue. d. Identical volcano plot as in (c) indicating autophagy related proteins (orange) and

564 MYOF and DYSF (red). e. Average peptide counts for the indicated proteins from $\mathrm{n}=3$ biological

565 replicates. f. Immunoprecipitation of purified lysosomes from the indicated cell lines showing

566 enrichment of MYOF and DYSF in PDA lysosome fractions. LAMP2 serves as a loading control

567 while absence of AIF, PDI and RCAS1 confirm organelle purity. g. Immunoblot showing levels of

568 MYOF and restricted expression of DYSF in the indicated human cell lines (PDA highlighted in

569 blue). h. Immuno-fluorescence staining of MYOF-HA (green) and LAMP2 (red) in HEK293T (left)

570 and KP4 (right) cells. Arrowheads indicate plasma membrane localization of MYOF in HEK293T

571 cells and lysosome localization in KP4 cells. Scale, $20 \mu \mathrm{m}$, inset scale, $2 \mu \mathrm{m}$. i. Biotinylation of cell

572 surface proteins in KP4 and HEK293T cells expressing MYOF-HA. Biotinylated proteins were

573 immuno-precipitated and western-blotted for MYOF. Note, MYOF is not on the cell surface of KP4

574 cells while MYOF-HA is present on the cell surface when expressed in HEK293T cells. j. Affinity

575 purified lysosomes were treated with increasing concentrations of Proteinase $\mathrm{K}$ as indicated.

576 Intraluminal proteins are protected from degradation (LAMP2, Cathepsin C, LC3B) while extra-

577 luminal proteins are sensitive to digestion (NPC1 and MYOF). 
Fig. 2 | PDA lysosomes are more resistant to lysosome membrane damage.

a

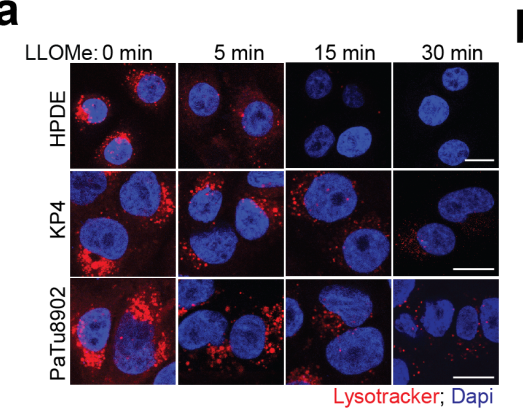

d

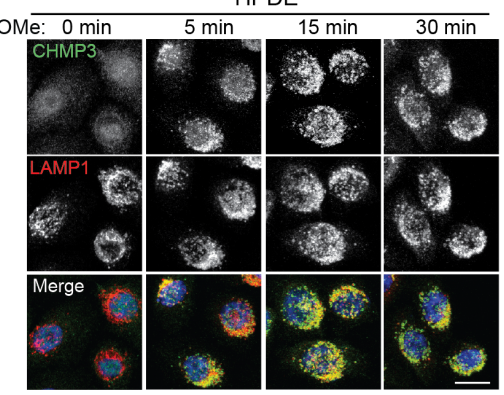

f

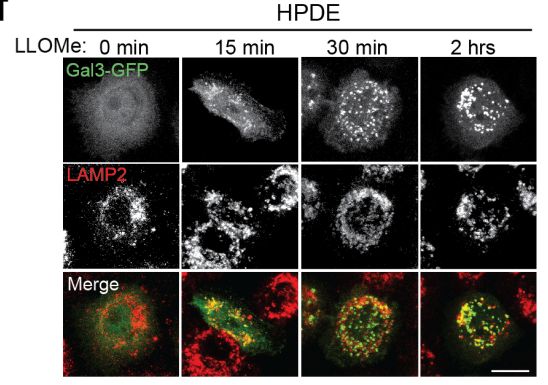

b
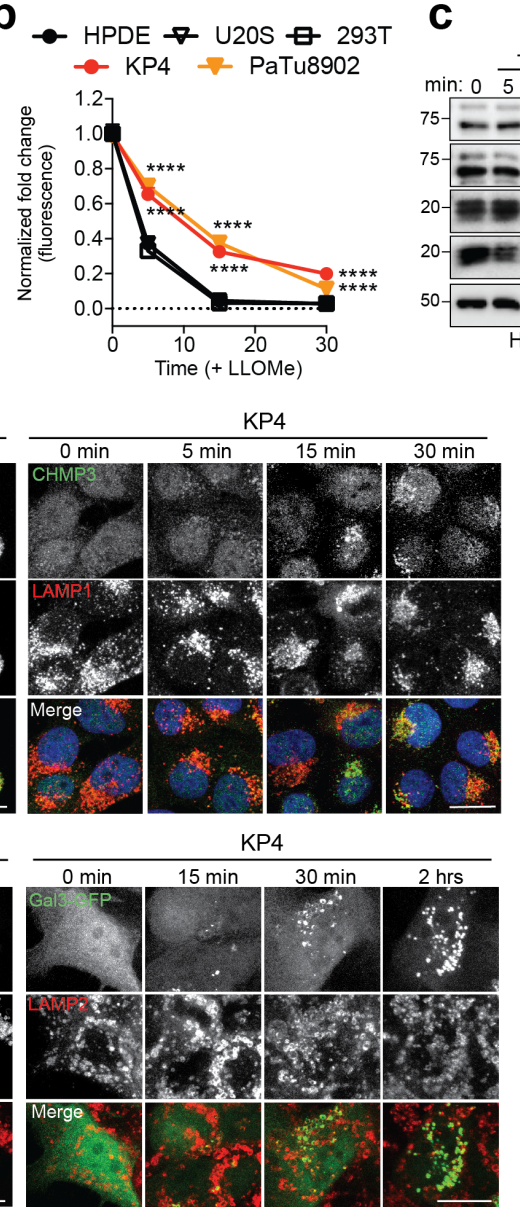

C

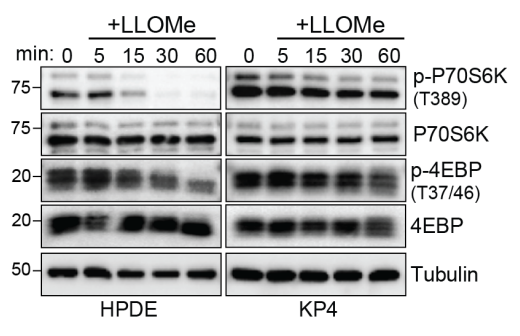

e

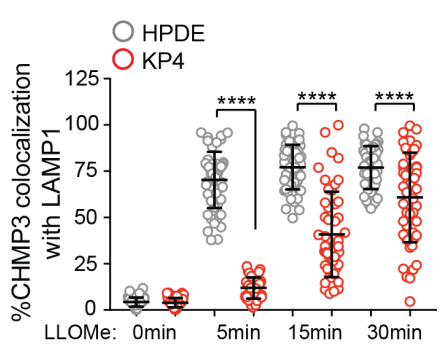

g

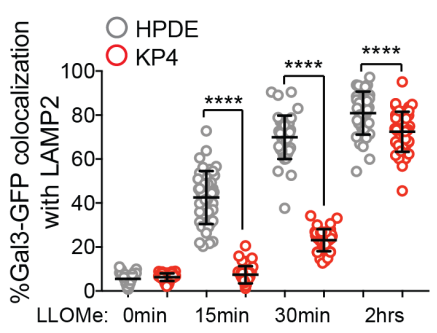

Fig. 2 | PDA lysosomes are more resistant to lysosome membrane damage.

a. Time-course of lysotracker red staining in HPDE, KP4 and PaTu8902 following treatment with LLOMe. (HPDE $n=76,80,81,80 ;$ KP4 $n=80,77,80$, 82; PaTu8902 n = 96, 96, 81, 82 cells). b. Quantification of normalized fold change in lysotracker red stain in HPDE, U20S, 293T, KP4 and PaTu8902 cells treated with LLOMe. Data are mean \pm s.e.m from $n=60-96$ cells per timepoint for each cell line. $P$ values determined by two-way ANOVA. c. Immunoblots for the indicated proteins in HPDE and KP4 cells following time course treatment with LLOMe. d. HPDE and KP4 cells with and without LLOMe treatment were co-stained for CHMP3 (green) and LAMP1 (red). e.

590 Quantification of percentage co-localization in control and LLOMe treated cells. (HPDE $n=50$, $59151,50,51$; KP4 n = 51, 50, 50, 50 cells). f. HPDE and KP4 cells expressing GFP-Galectin 3 592 (green) were treated with LLOMe for the indicated times and co-stained for LAMP2 (red). g. 593 Quantification of percentage co-localization in control and LLOMe treated cells. (HPDE $\mathrm{n}=39$, 
bioRxiv preprint doi: https://doi.org/10.1101/2021.01.04.425106; this version posted January 4, 2021. The copyright holder for this preprint

(which was not certified by peer review) is the author/funder, who has granted bioRxiv a license to display the preprint in perpetuity. It is made available under aCC-BY-NC-ND 4.0 International license.

$59443,40,42 ; \mathrm{KP} 4 \mathrm{n}=43,42,40,42$ cells). Scale, $20 \mu \mathrm{m}$. Data are mean \pm s.d. $P$ values determined

595 by unpaired two-tailed $t$-tests. ${ }^{* * *} P<0.0001$. 
bioRxiv preprint doi: https://doi.org/10.1101/2021.01.04.425106; this version posted January 4, 2021. The copyright holder for this preprint (which was not certified by peer review) is the author/funder, who has granted bioRxiv a license to display the preprint in perpetuity. It is made available under aCC-BY-NC-ND 4.0 International license.

Fig. 3 | MYOF is essential for lysosome function in PDA cells.

a

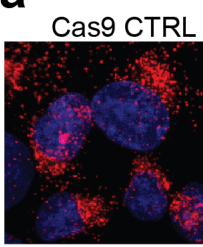

KP4
KO \#1

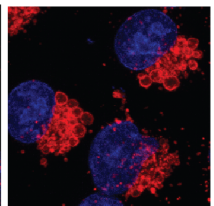

28.

-

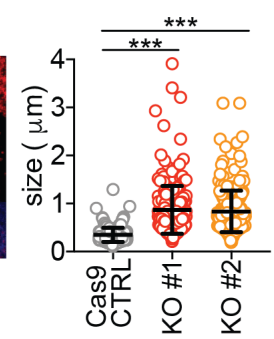

b
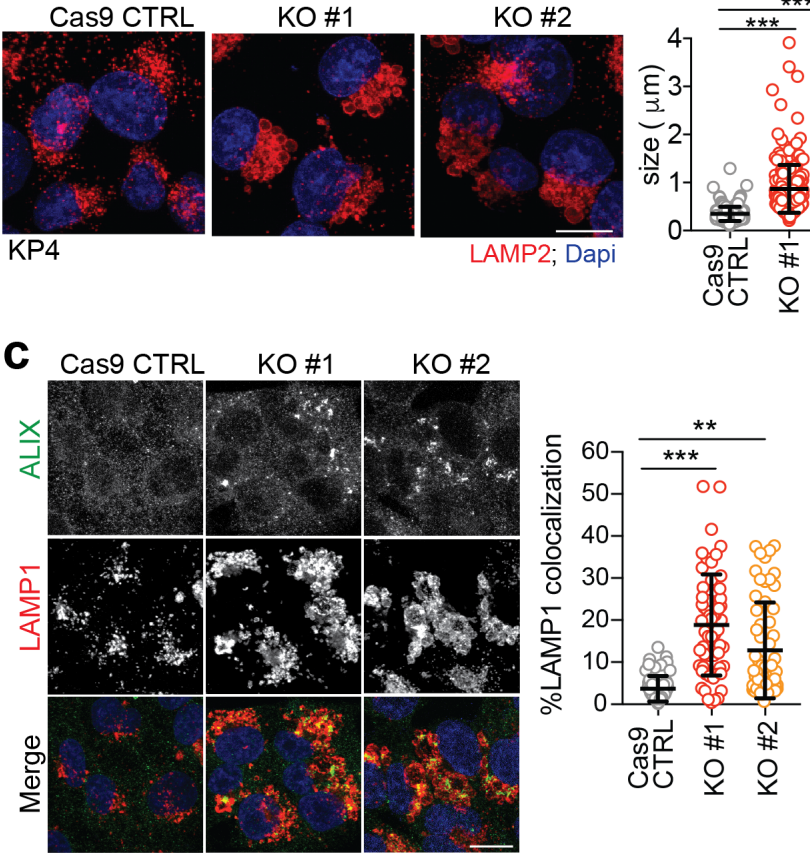

d Cas9 CTRL

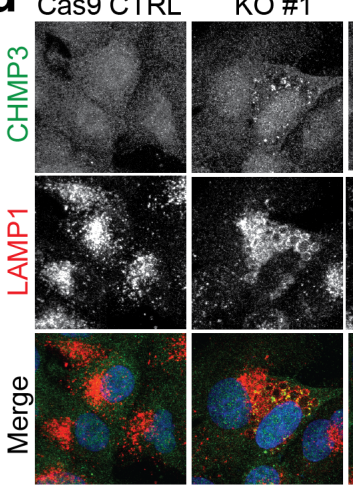

$\mathrm{KO} \# 2$
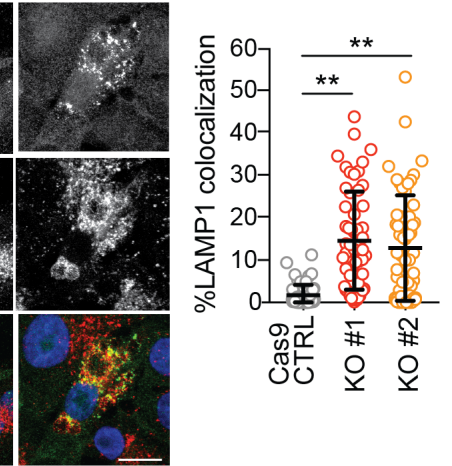

e

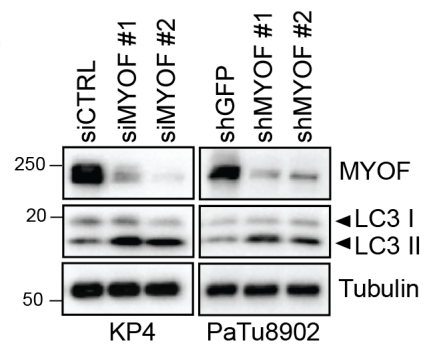

f

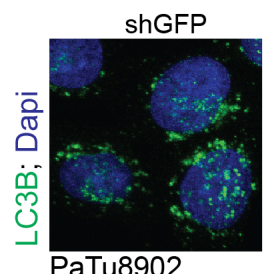

PaTu8902
ShMYOF \#1

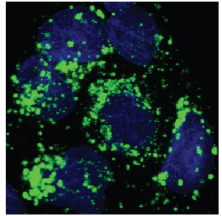

$2,2=0$

LC3B; Dapi

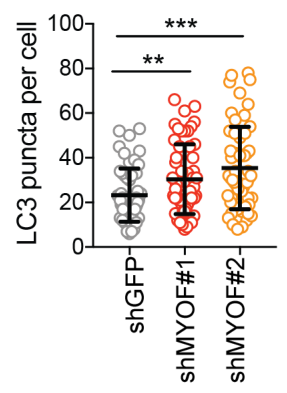

i

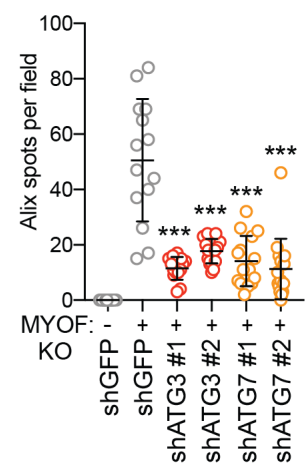

开留留

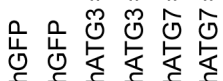

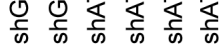

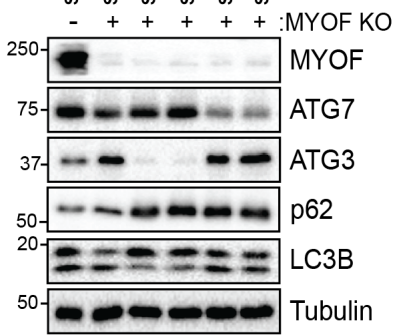

MYOF KO
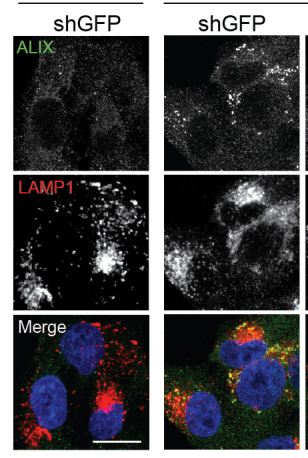

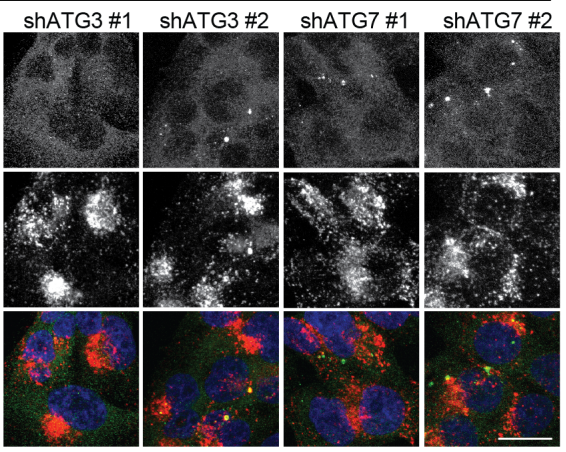

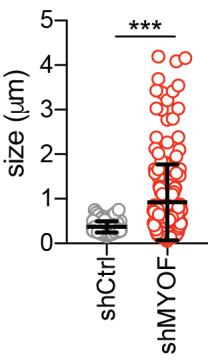




\section{Fig. 3 | MYOF is essential for lysosome function in PDA cells.}

598 a. Immunofluorescence staining of LAMP2 (red) following CRISPR mediated knockout of MYOF 599 and Cas9 control KP4 cells. Graph at right shows measurement of lysosome diameter from $\mathrm{n}=$ 600252 (Cas9 control), 251 (KO\#1, KO\#2). b. Representative electron microscopy images showing 601 aberrant lysosome morphology in KP4 cells following shRNA mediated knockdown of MYOF. 602 Arrowheads highlight differential lysosome morphology in shGFP versus shMYOF conditions. 603 Graph on the right shows quantification of lysosome diameter ( $n=200$ lysosomes from control; $n$ $604=202$ lysosomes from MYOF KD cells). Scale bar $1 \mu \mathrm{m}$. c, d. Increased recruitment of ALIX 605 (green) (f) and CHMP3 (green) (g) to LAMP1 positive lysosomes (red) following KO of MYOF in 606 KP4 cells relative to Cas9 control cells. Graphs show the quantification of percentage ALIX ( $\mathrm{n}=$ 607 60) and CHMP3 ( $\mathrm{n}=57$ ) co-localization with LAMP1. e. Immunoblot showing increased LC3B 608 lipidation (arrowheads) in KP4 and 8902 cells upon siRNA or shRNA mediated knockdown of 609 MYOF. f. Immunofluorescence staining for LC3B (green) showing increase accumulation of LC3B 610 positive autophagosomes in KP4 cells following shRNA mediated knockdown of MYOF compared 611 to control cells. i. Graph on the right shows quantification of LC3B puncta ( $\mathrm{n}=14$ fields/condition).

612 g. Immunoblot confirming shRNA mediated knockdown of ATG3 and ATG7 and autophagy 613 blockade in KP4 MYOF KO cells. $\mathbf{h}$. Recruitment of ALIX (green) to lysosomes (LAMP1; red) in 614 KP4 cells in the presence (WT; $n=14)$ and absence $(K O ; n=14)$ of MYOF. shRNA mediated 615 knockdown of ATG3 $(n=14,15)$ or ATG7 $(n=15,16)$ to suppress autophagy causes a decrease 616 in lysosome localization of ALIX in MYOF KO cells. i. Graph on the right shows quantification of 617 ALIX puncta per condition. Scale, $20 \mu \mathrm{m}$. Data are mean \pm s.d. $P$ values determined by unpaired 618 two-tailed $t$-tests. ${ }^{* *} P<0.01 ;{ }^{* * *} P<0.001$. 
Fig. 4 | The $\mathbf{N}$ Terminal C2 domains of MYOF are required for membrane protection.

a $M Y O F(W T)$

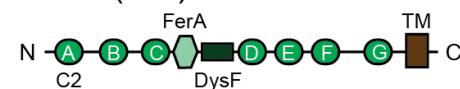

MYOF-FRB*

-A-B-OC=D-B-C-OA-M

MYOF $\triangle$ C2-FRB*

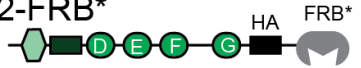

C

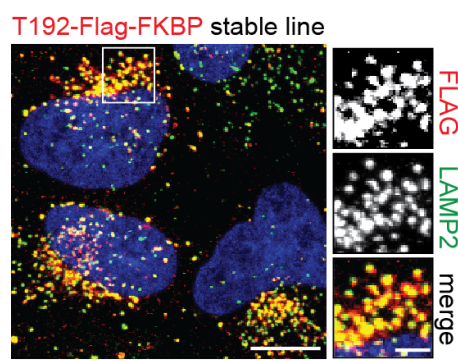

e

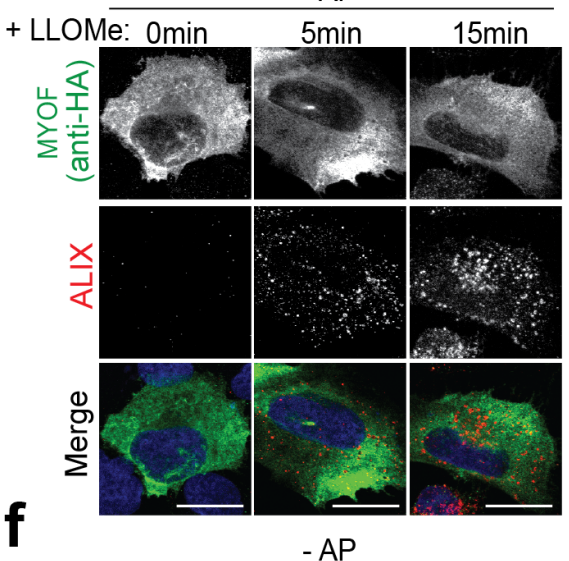

+ LLOMe: 0 min $\quad 5 \min \quad 15 \mathrm{~min}$
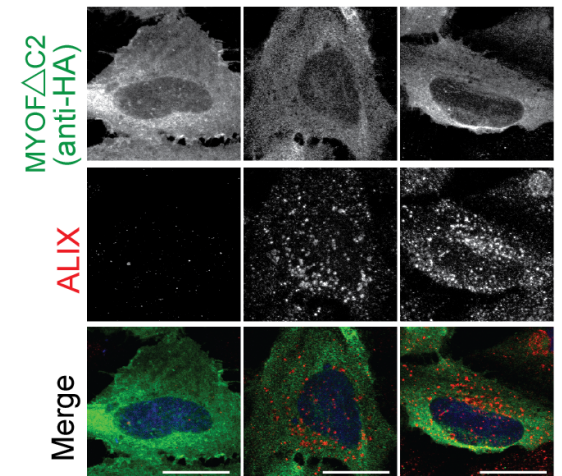

b

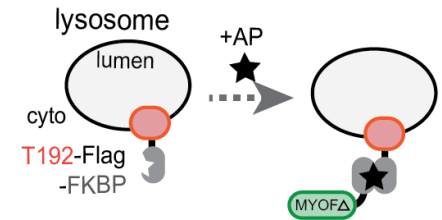

\section{MYOF}

d
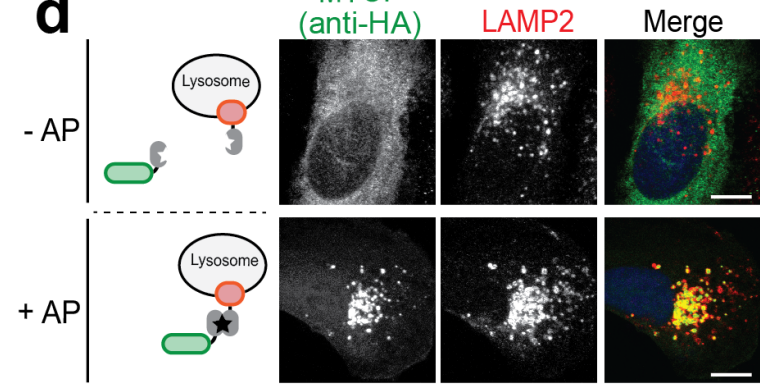
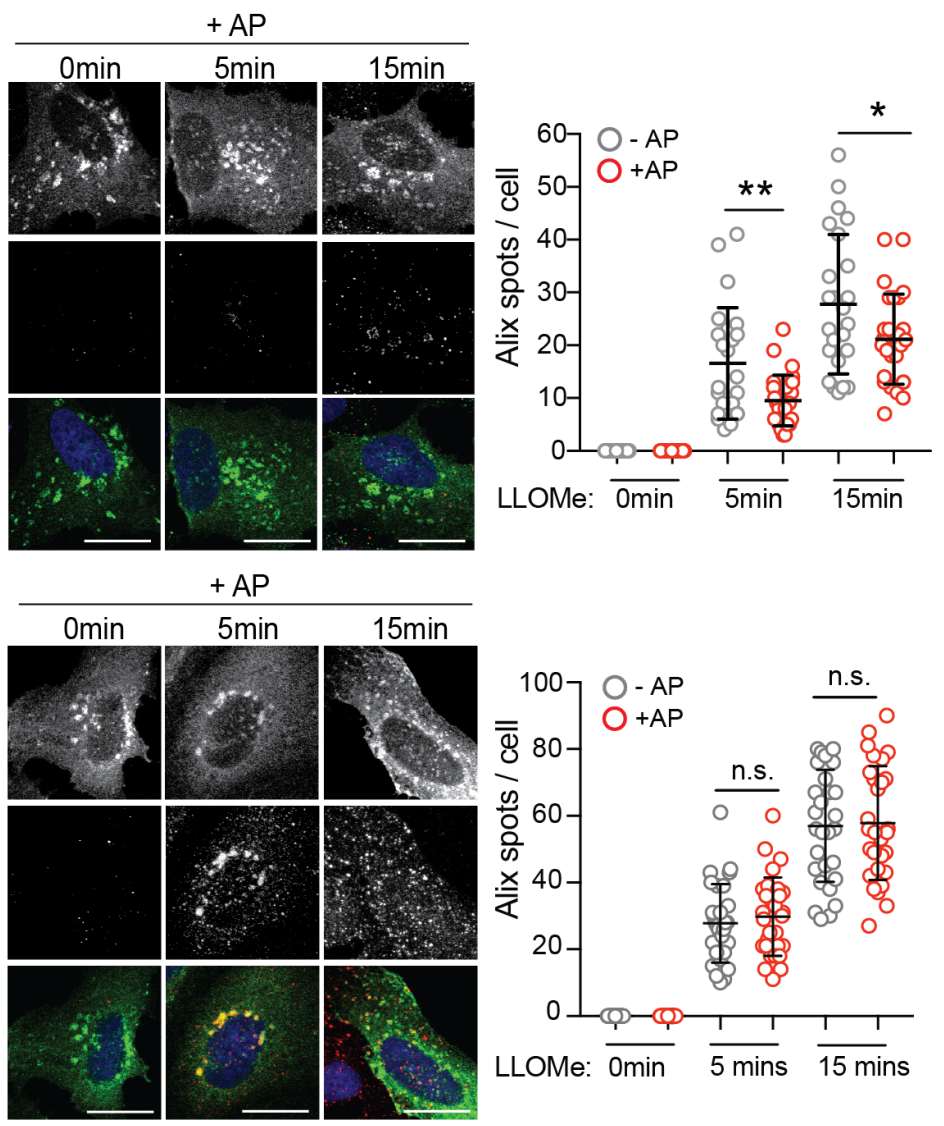
621 Fig. 4 | The $\mathbf{N}$ terminal $\mathbf{C 2}$ domains of MYOF are required for membrane protection.

622 a. Domain structure of MYOF and MYOF-FRB* variants. b. Schematic showing

623 heterodimerization of MYOF-FRB* to lysosome membrane anchored T192-Flag-FKBP following

624 addition of the rapalogue (AP21967; AP). TM, transmembrane domain. c. Lysosomal localization

625 of T192-Flag-FKBP (Flag) in stably expressing U20S cells. d. Transient expression of MYOF-

626 FRB* (MYOF; detected with anti-HA antibody) in U20S cells stably expressing T192-Flag-FKBP.

627 In the absence of AP, MYOF-FRB* is cytoplasmic while upon AP addition MYOF $\triangle T M$ is recruited

628 to LAMP2 positive lysosomes (red). e. Recruitment of MYOF-FRB* protects against LLOMe

629 induced damage and ALIX recruitment. U20S-T192-Flag-FKBP expressing cells transfected with

630 MYOF-FRB* were treated with LLOMe for the indicated time points in the absence $(n=30,24$,

63124 cells) or presence ( $n=30,27,27$ cells) of AP, followed by immuno-staining for HA (green) and

632 ALIX (red). f. U20S-T192-Flag-FKBP cells transfected with MYOF $\triangle$ C2-FRB were treated as in 'e'

633 (- AP $n=29$ cells; + AP $n=29$ cells) followed by immuno-staining for HA (green) and ALIX (red).

634 Lysosomal recruitment of MYOF $\Delta$ C2 does not protect against LLOMe induced ALIX recruitment.

635 Graphs at right show quantification of ALIX spots per cell in response to LLOMe. Scale for all

636 panels, $20 \mu \mathrm{m}$. Data are mean \pm s.d. $P$ values determined by unpaired two-tailed $t$-tests. ${ }^{*} P<$

$637 \quad 0.05 ;{ }^{* *} P<0.01 ;$ n.s. not significant. 
Fig. 5 | MYOF is required for PDA tumor growth

a b

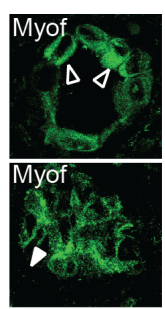

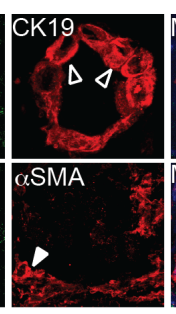

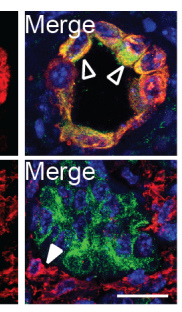

f
C
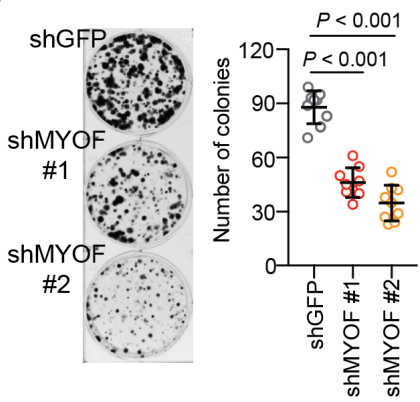
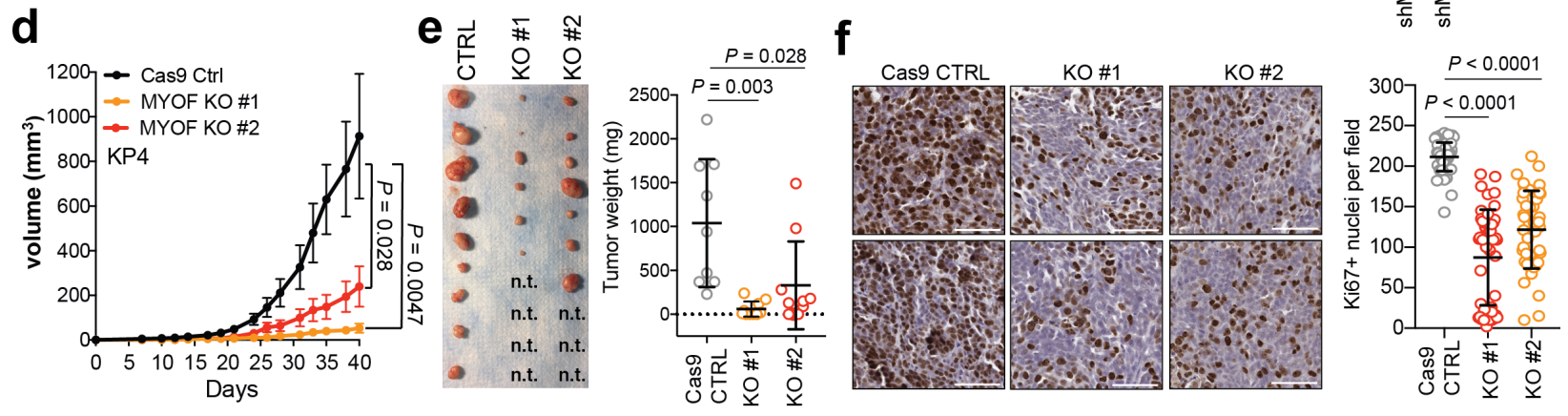

Fig. 5 | MYOF is required for PDA tumor growth.

a. Expression levels of Myof and Dysf in mouse KPC derived PDA tumours relative to normal liver, normal pancreas and the mouse myoblast cell line, C2C12. b. Immuno-fluorescence staining of Myof (green), the epithelial marker CK19 (red; top) and the stromal marker $\alpha$-SMA (red, bottom) in mouse KPC tumours showing co-localization of Myof with CK19 (open arrowheads) but not $\alpha$ SMA (close arrow heads). Scale, $50 \mu \mathrm{m}$. c. Colony formation of shGFP or shMYOF infected KP4 cells. Graph shows quantification of colony area per condition from $n=9$ independent experiments. d. In vivo growth in nude mice of s.c. KP4 xenografts following CRISPR mediated KO of MYOF. $\mathrm{N}=9$ (CTRL), 10 (KO\#1), 10 (KO\#2) tumours per group. Error bars represent s.e.m. e. Images (left) and tumour weight (right) of control and MYOF KO KP4 xenografts resected at day 42. n.t. no macroscopic tumour identified upon resection. f. Ki67 staining of control and MYOF KO xenografts. Graph shows quantification of Ki67 positive nuclei from $n=56$ (control), 46 (KO\#1), 46 (KO\#2) fields from 3-4 tumours per group. Scale, 100 $\mu \mathrm{m}$. 
Fig. 6 | High MYOF expression levels correlate with aggressive disease

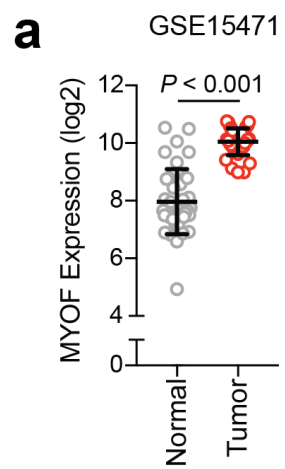

(38) (38)

GSE16515

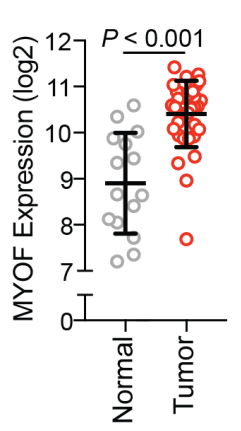

(15) (36)

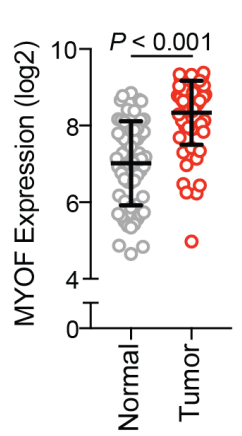

(61) (69)

GSE28735

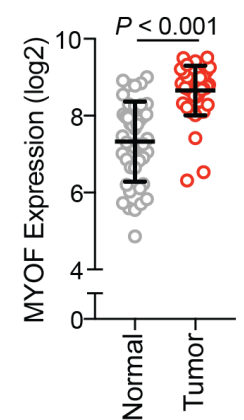

(45) (45)

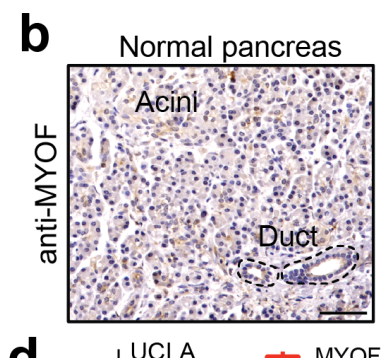

d

e
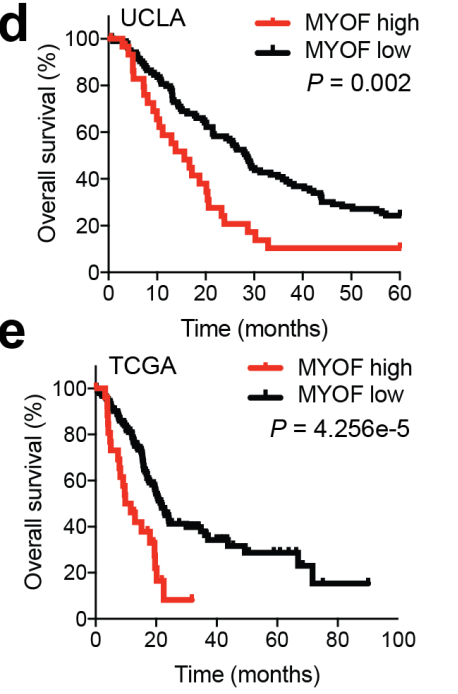

Primary tumor

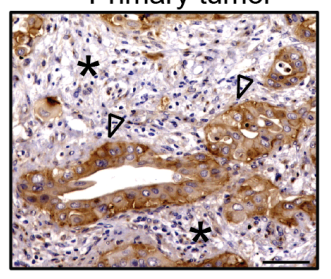

f
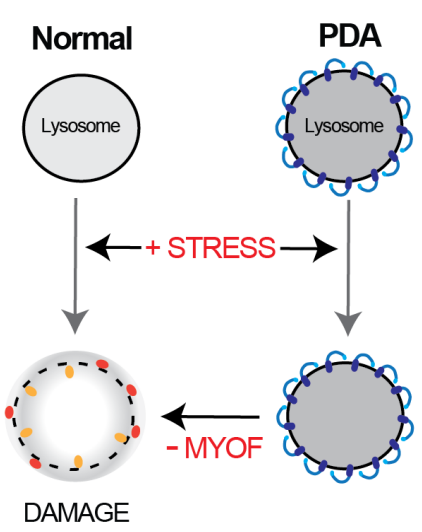

$\frown$ MYOF ESCRT GAL3

Fig. 6 | High MYOF expression levels correlate with aggressive disease

a. MYOF transcript levels in human PDA specimens and normal pancreas (adjacent nonneoplastic tissue) from the indicated datasets. b. Immuno-histochemistry showing increased expression of MYOF in primary patient PDA tumour epithelia (arrowheads) compared to normal pancreas or adjacent stroma (asterisk). Scale, $100 \mu \mathrm{m}$. c. Percentage distribution of semiquantitative histoscore of MYOF staining across normal adjacent $(n=102)$ and primary PDA $(n=$ 136). d, e. High expression of MYOF predicts shorter overall survival in two patient cohorts. $N=$ 136 patients in the UCLA cohort (MYOF high $n=31$, MYOF low $n=105$ ) and $n=185$ in The Cancer Genome Atlas (TCGA) cohort (MYOF high; $Z$ score $>1, n=27$; MYOF low $Z$ score $<1, n=158$ ). p-Value calculated by Log-rank test. f. Model comparing lysosomal response to stress in normal (left) and PDA (right) cells. Lysosomal retargeting of MYOF in PDA cells provides protection against membrane stress caused by increased rates of vesicular traffic. Loss of MYOF renders PDA lysosomes more vulnerable to damage. Data are mean \pm s.d. $P$ values determined by unpaired two-tailed $t$-tests. 


\section{Supplementary. Fig. 1 | MYOF is a novel lysosomal membrane protein in PDA cells.}

a

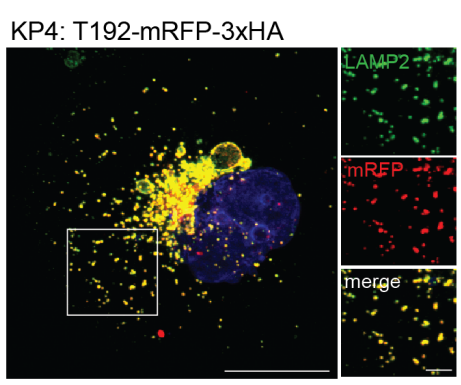

b

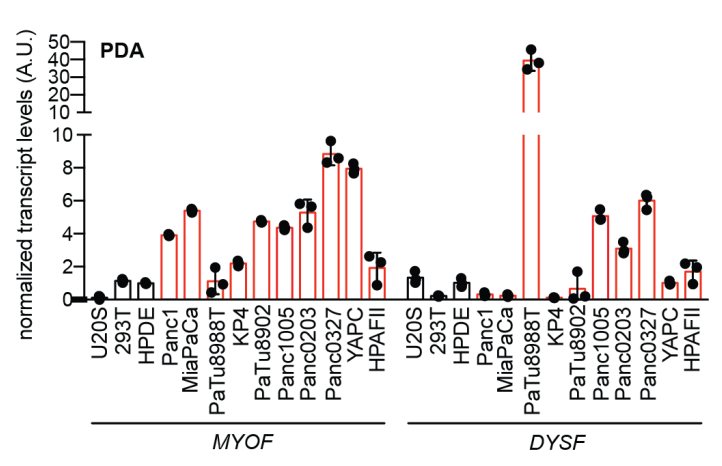

C MiaPaca (PDA)

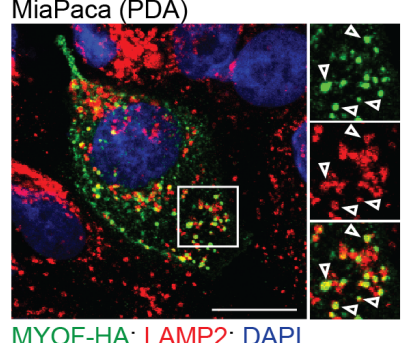

PaTu8902 (PDA)

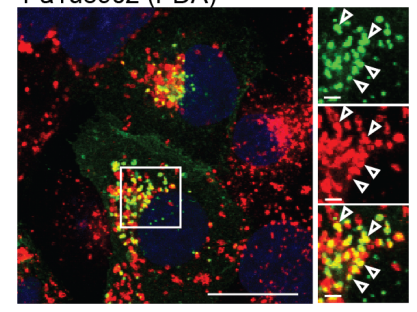

MYOF-HA: LAMP2; DAPI

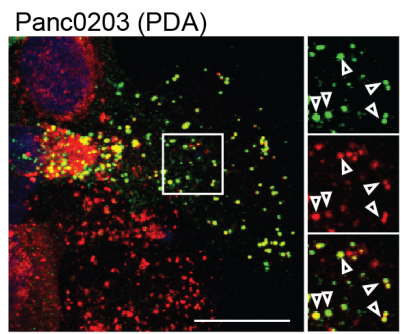

MYOF-HA: LAMP2; DAPI

d
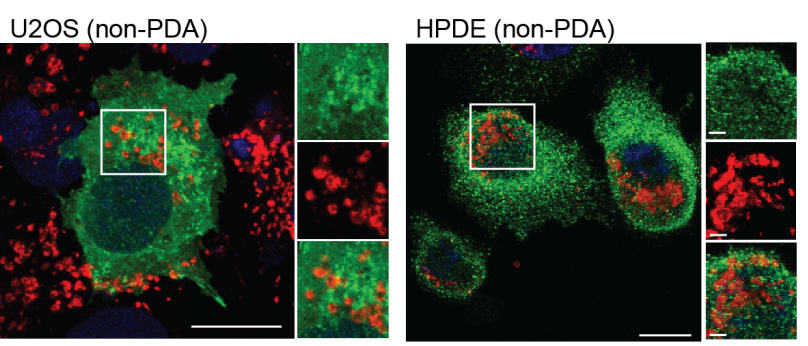

MYOF-HA: LAMP2; DAP

MYOF-HA: LAMP2; DAPI

e $\quad$ - p $62-$ Myoferlin

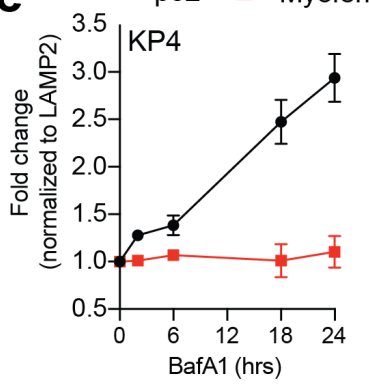

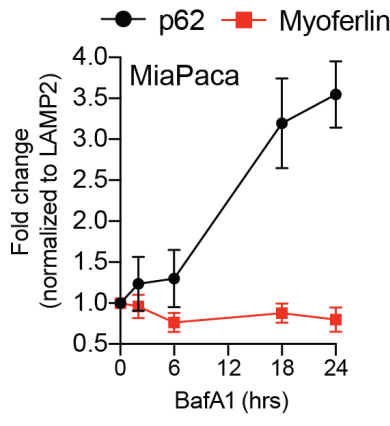

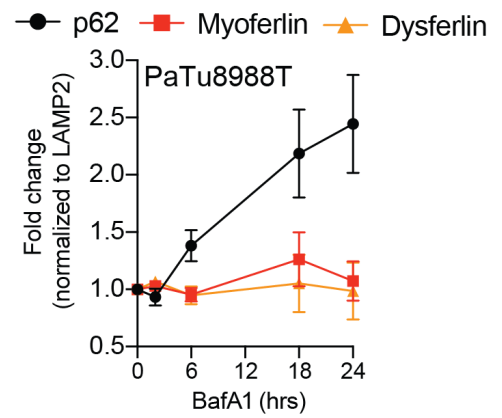

671 Supplementary Figure 1 | MYOF is a novel lysosomal membrane protein in PDA cells.

672 a. Co-localization of T192-mRFP-3xHA and endogenous LAMP2 in KP4 cells. b. qRT-PCR

673 analysis of MYOF and DYSF mRNA levels across 10 human PDA cell lines. c,d. Immuno-

674 fluorescence staining of MYOF-HA (green) and LAMP2 (red) in PDA cell lines (MiaPaca,

675 Patu8902 and Panc0203) (c) and non-PDA (U2OS and HPDE) cell lines (d). Arrowheads show 
676 examples of co-localization. e. Treatment of KP4, MiaPaCa and PaTu8988T cells with 75nM

677 BafA1 for the indicated times causes an increase in p62 levels but not MYOF or DYSF. Graph

678 shows the quantification of normalized fold change relative to LAMP1, averaged from 3

679 independent experiments. Scale, $20 \mu \mathrm{m}$ for all panels. 
bioRxiv preprint doi: https://doi.org/10.1101/2021.01.04.425106; this version posted January 4, 2021. The copyright holder for this preprint (which was not certified by peer review) is the author/funder, who has granted bioRxiv a license to display the preprint in perpetuity. It is made available under aCC-BY-NC-ND 4.0 International license.

\section{Supplementary. Fig. 2 | PDA lysosomes are resistant to multiple membrane perturbing agents.}

\section{a}

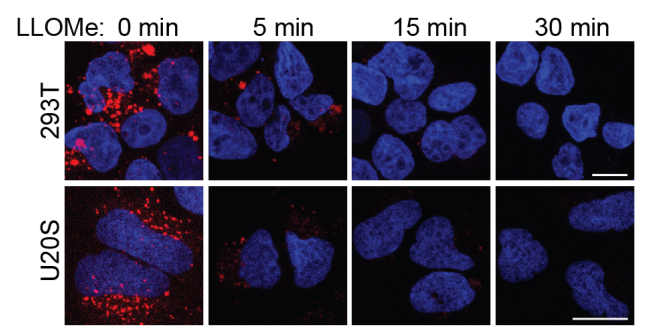

C

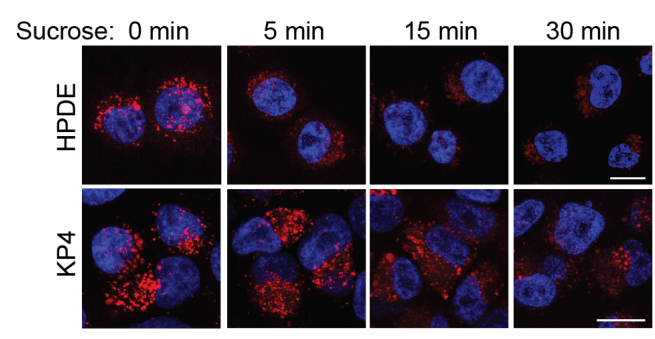

e
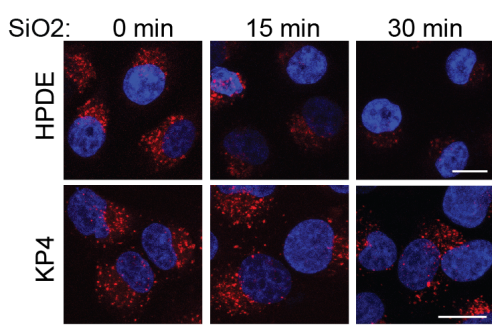

b

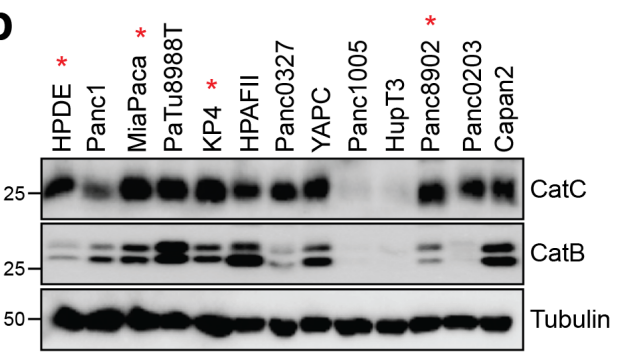

d

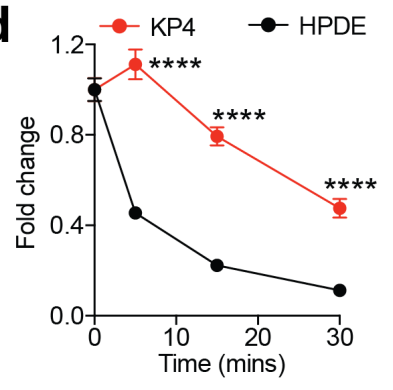

f

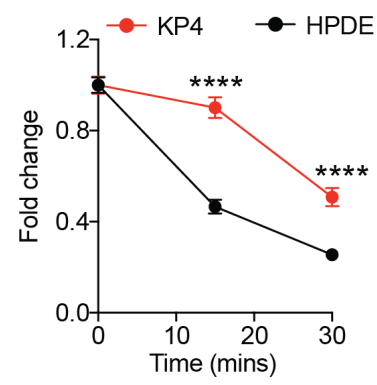

g

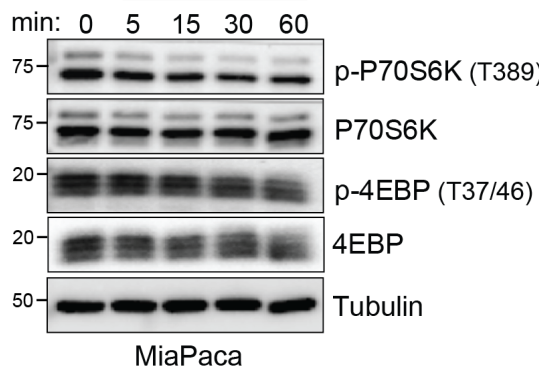

h

ชำ ำก

$8 \stackrel{\circ}{\circ}$ กึ

$8 \simeq \stackrel{2}{0}$ LLOMe 00000.

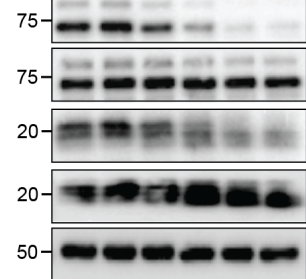

HPDE

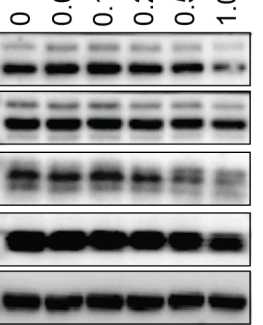

KP4 
681 Supplementary Figure 2 | PDA lysosomes are resistant to multiple membrane perturbing 682 agents.

683 a. Time-course of lysotracker red staining in 293T and U20S cells following treatment with 684 LLOMe. (293T, $\mathrm{n}=80$ cells per time point; U20S, $\mathrm{n}=57$ - 63 cells per time point). b. Immunoblot 685 showing the expression of Cathepsin $C$ and Cathepsin B in the indicated cell lines. Asterisk 686 denotes cell lines used throughout the study. c. Time-course of lysotracker red staining in HPDE 687 and KP4 cells following treatment with 0.5M sucrose. (HPDE, $n=64$ cells per time point; KP4, $n$ $688=63$ cells per time point). $\mathbf{d}$. Normalized fold change of lysotracker staining. e. Time-course of 689 lysotracker red staining in HPDE and KP4 cells following treatment with $100 \mu \mathrm{g} / \mathrm{ml}$ silica. (HPDE, $690 \mathrm{n}=65$ cells per time point; KP4, $\mathrm{n}=65$ cells per time point). f. Normalized fold change of 691 lysotracker staining. g. Immunoblots for the indicated proteins in MiaPaca cells following a time 692 course of $1 \mathrm{mM}$ LLOMe treatment. $\mathbf{h}$. Immunoblots for the indicated proteins in HPDE, KP4 and 693 MiaPaca cells following treatment for $1 \mathrm{hr}$ with increasing doses of LLOMe. Scale, $20 \mu \mathrm{m}$ for all 694 panels. Data are mean \pm s.d. $P$ values determined by unpaired two-tailed $t$-tests. ${ }^{* * *} P<0.0001$. 
bioRxiv preprint doi: https://doi.org/10.1101/2021.01.04.425106; this version posted January 4, 2021. The copyright holder for this preprint (which was not certified by peer review) is the author/funder, who has granted bioRxiv a license to display the preprint in perpetuity. It is made available under aCC-BY-NC-ND 4.0 International license.

Supplementary. Fig. 3 | Recruitment of ESCRT proteins to PDA lysosomes is delayed following acute damage

a

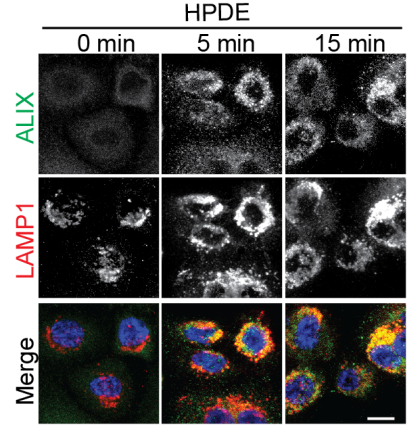

b
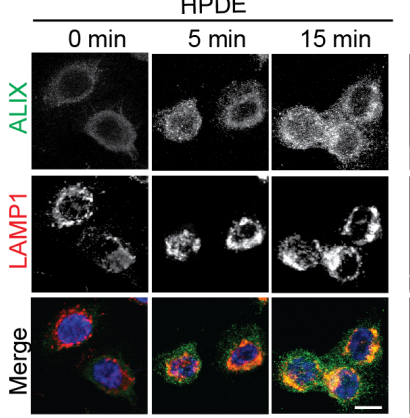

C

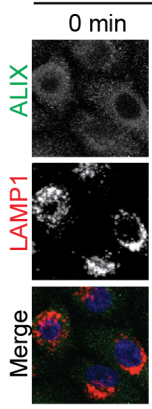

d

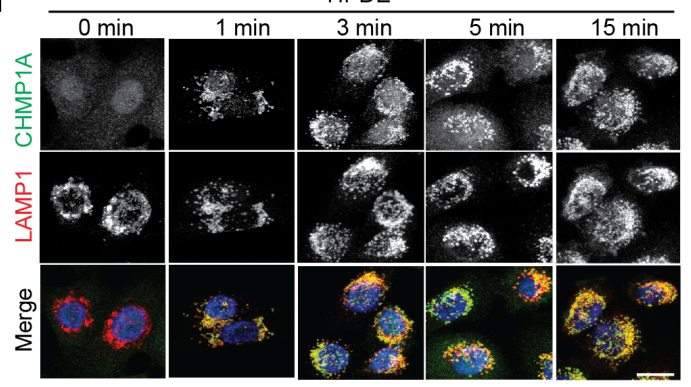

$\mathbf{e}$

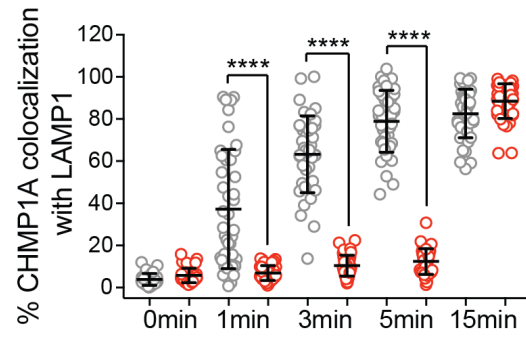

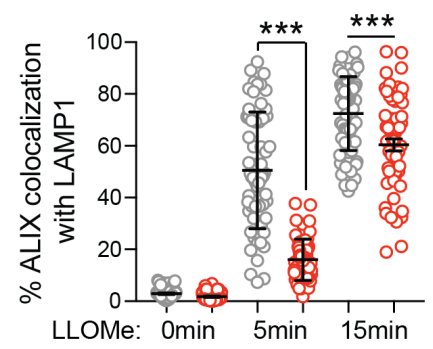

O HPDE
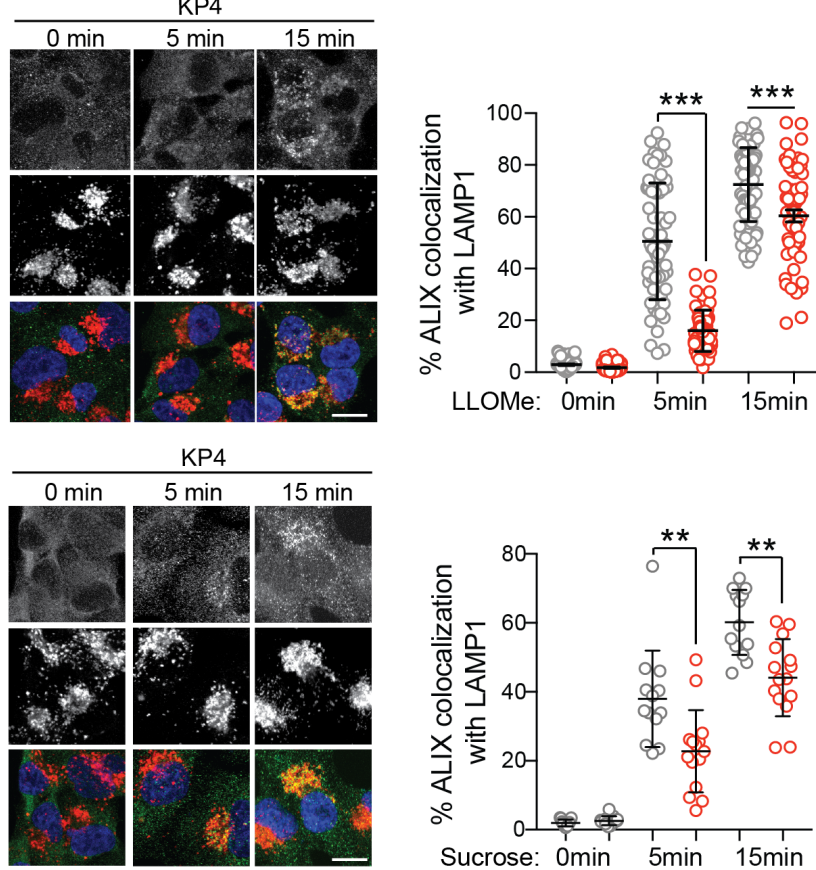

O HPDE

O HPDE $\quad \mathbf{f}$

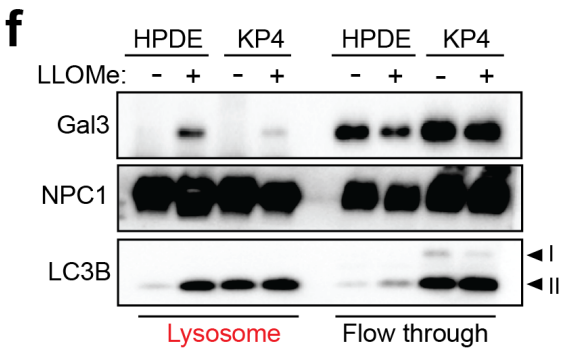

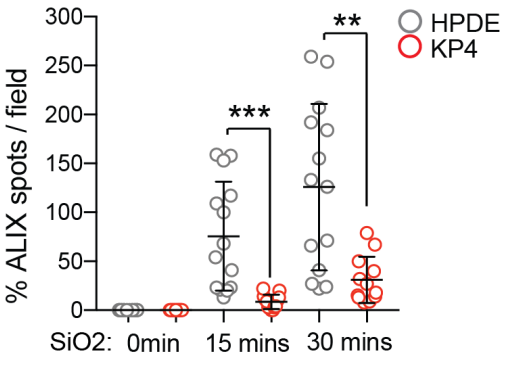

romin
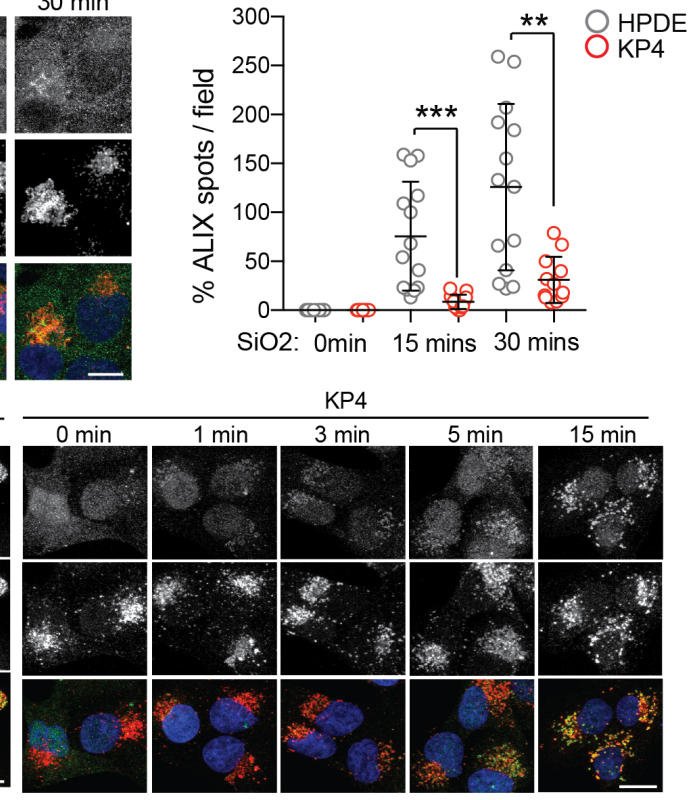

SiO2: 0 min 15 mins 30 mins 
696 Supplementary Figure 3 | Recruitment of ESCRT proteins to PDA lysosomes is delayed

697 following acute damage.

698 a-c. Time course of LLOMe (a), 0.5M sucrose (b), $100 \mu \mathrm{g} / \mathrm{ml}$ silica (c) treatment of HPDE and

699 KP4 cells followed by immuno-fluorescence staining for ALIX (green) and LAMP1 (red). Graphs

700 show the quantification of percentage co-localization of ALIX (LLOMe, $n=60$; sucrose, $n=13-15$

701 fields/conditions; silica, $n=12-14$ fields/condition per cell line) with LAMP1 positive lysosomes.

702 d. Time course of LLOMe treatment of HPDE and KP4 cells followed by immuno-fluorescence

703 staining for CHMP1A (green) and LAMP1 (red). e. Graph shows quantification of percentage co-

704 localization of CHMP1A ( $\mathrm{n}=40$ per cell line) with LAMP1 positive lysosomes. $\mathrm{f}$. Immunoblot for

705 the indicated proteins in lysosome fractions and flow through fractions isolated from HPDE- and

706 KP4-T192-mRFP-3xHA stable cell lines treated with LLOMe for 10min. Scale, $20 \mu \mathrm{m}$. Data are

707 mean \pm s.d. $P$ values determined by unpaired two-tailed $t$-tests. ${ }^{* *} P<0.01 ;{ }^{* * *} P<0.001 ;{ }^{* * *} P$

$708<0.0001$. 
bioRxiv preprint doi: https://doi.org/10.1101/2021.01.04.425106; this version posted January 4, 2021. The copyright holder for this preprint (which was not certified by peer review) is the author/funder, who has granted bioRxiv a license to display the preprint in perpetuity. It is made available under aCC-BY-NC-ND 4.0 International license.

Supplementary. Fig. 4 | MYOF loss leads to lysosome dysfunction in PDA cells.

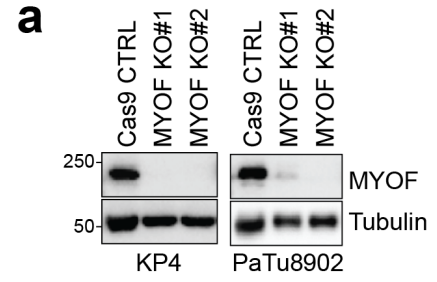

C

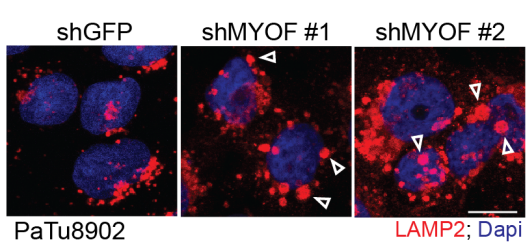

e

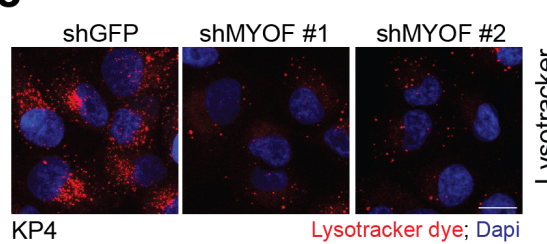

g

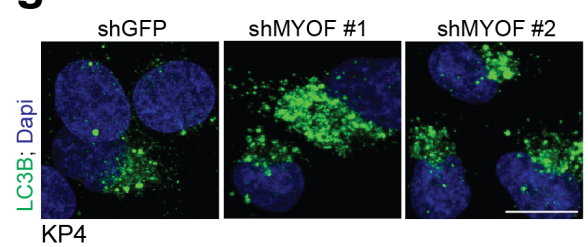

$\mathbf{h}$

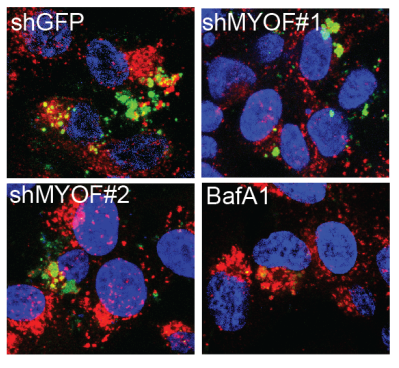

b
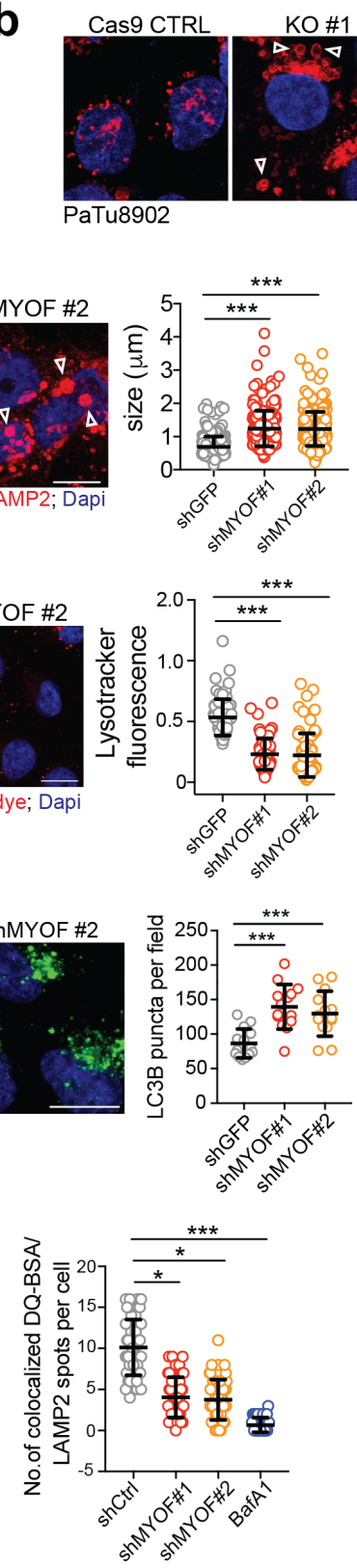

$\mathrm{KO} \# 1$
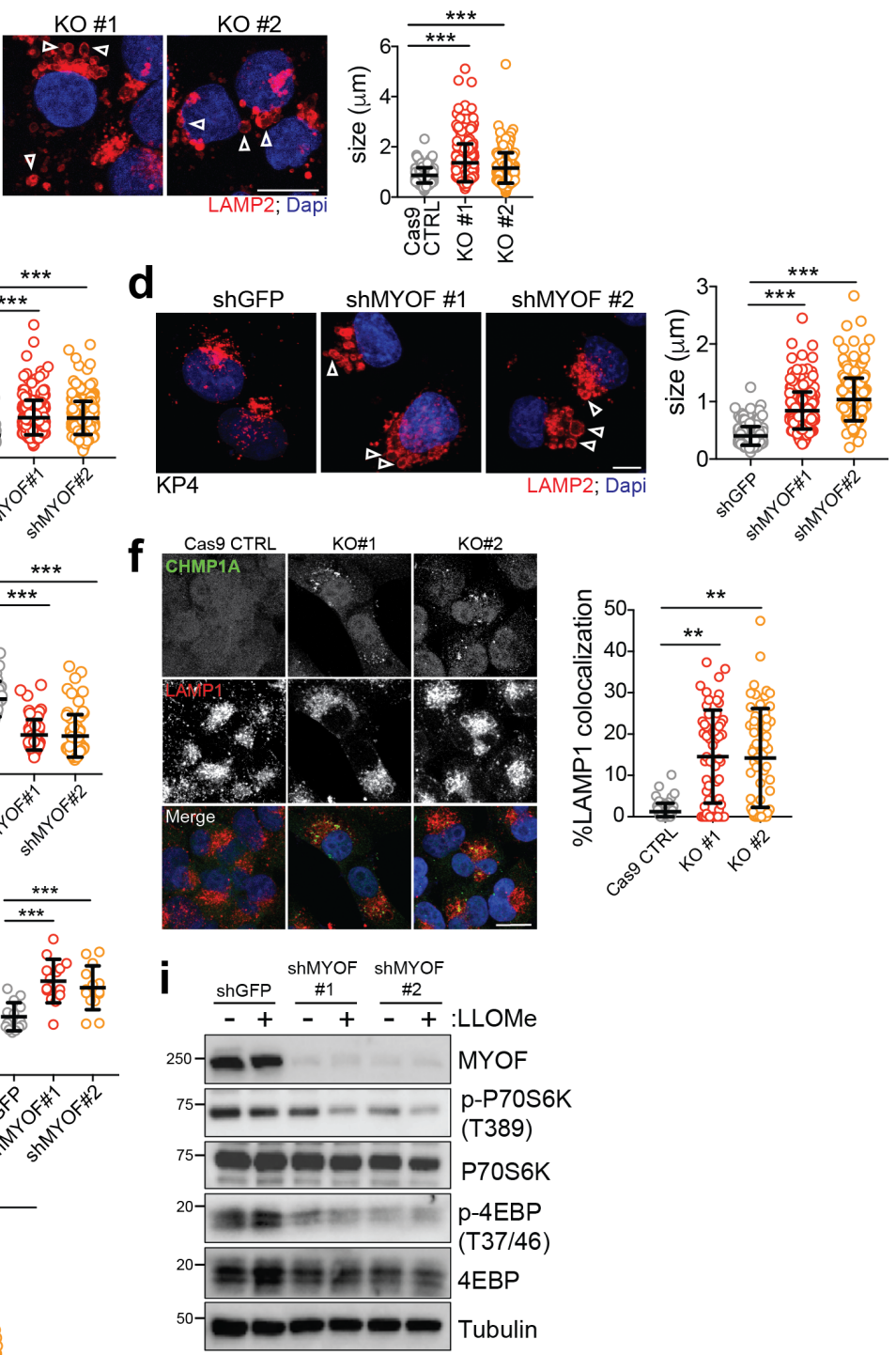
710 Supplementary Figure 4 | MYOF loss leads to lysosome dysfunction in PDA cells.

711 a. Immunoblot showing the efficiency of MYOF ablation in KP4 and PaTu8902 CRISPR KO

712 clones. b. MYOF KO in PaTu8902 cells causes aberrant lysosomal morphology and increased

713 size as shown by LAMP2 staining (arrowheads). Graph on the right shows the measurement of

714 lysosome diameter in Cas9 control cells $(n=252)$, MYOF KO \#1 $(n=255)$ and MYOF KO \#2 ( $n$

715 = 258). $\mathbf{c}$, d. shRNA mediated knockdown of MYOF in PaTu8902 (c) and KP4 (d) cells leads to

716 defects in lysosome morphology and size as shown by LAMP2 staining (arrowheads). Graphs on

717 the right show the measurement of lysosome diameter [8902 shGFP $(n=258)$, shMYOF\#1 $(n=$

718 256), shMYOF\#2 ( $n=263)$; KP4 shGFP $(n=254)$, shMYOF\#1 $(n=239)$, shMYOF\#2 $(n=243)$

719 cells]. e. Lysotracker staining in KP4 cells following infection with shRNA against GFP or MYOF.

720 Graph on the right shows the quantification of normalized fold change in lysotracker fluorescence

721 in control (shGFP, $\mathrm{n}=70$ cells) and KD (shMYOF\#1, $\mathrm{n}=65$; shMYOF\#2, $\mathrm{n}=77$ ) conditions. f.

722 Increased recruitment of CHMP1A (green) to LAMP1 positive lysosomes (red) following KO of

723 MYOF (\#1, $n=60 ; \# 2, n=61)$ in KP4 cells relative to Cas9 control $(n=58)$ cells. Graph shows

724 the quantification of percentage CHMP1A co-localization with LAMP1. g. Immuno-fluorescence

725 staining for LC3B in PaTu8902 cells following infection with shGFP ( $\mathrm{n}=55$ cells) or shRNA

726 mediated knockdown of MYOF (\#1, $\mathrm{n}=57 ; \# 2, \mathrm{n}=58$ cells). Graph on the right shows the

727 quantification of LC3B puncta. h. Proteolysis of macropinocytosed protein is impaired following

728 shRNA mediated knockdown of MYOF or BafA1 treatment as determined by pulse-chase with

729 DQ-BSA. Degradation of DQ-BSA in lysosomes is quantified (number of fluorescent spots/cell co-

730 localizing with LAMP2 positive lysosomes from $n=54$ (shGFP), 57 (shMYOF\#1), 52 (shMYOF\#2),

73154 (BafA1 treated) cells. i. Immunoblots for the indicated proteins in KP4 control cells (shGFP) or

732 following shRNA mediated knockdown of MYOF, with or without $1 \mathrm{mM}$ LLOMe treatment to

733 determine mTORC1 signaling activity. Data are mean \pm s.d. Scale, $20 \mu \mathrm{m}$ for all panels. $P$ values

734 determined by unpaired two-tailed $t$-tests. ${ }^{*} P<0.05 ;{ }^{* *} P<0.01$; ${ }^{* * *} P<0.001 ;{ }^{* * *} P<0.0001$. 
Supplementary. Fig. 5 | Lysosomal targeting of MYOF delays onset of membrane damage.

a

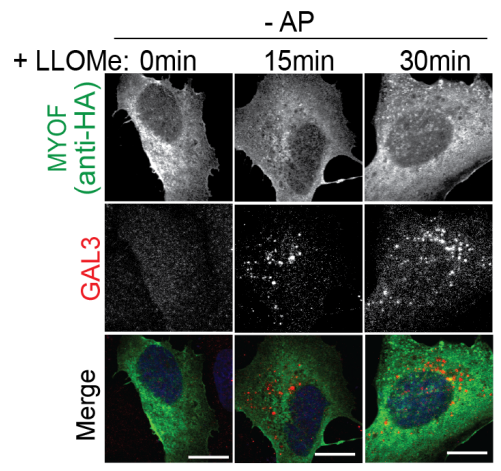

b

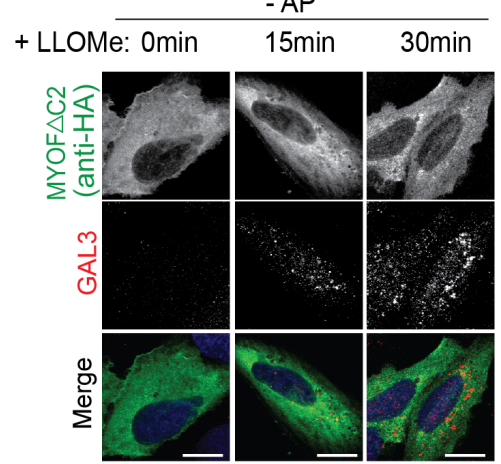

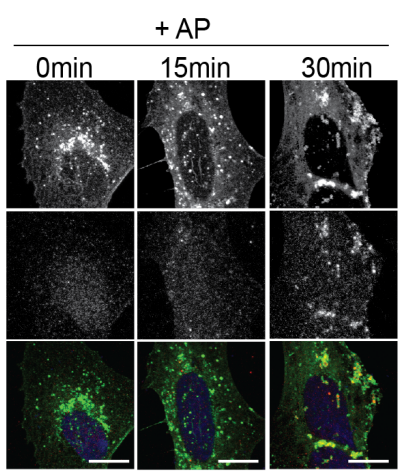
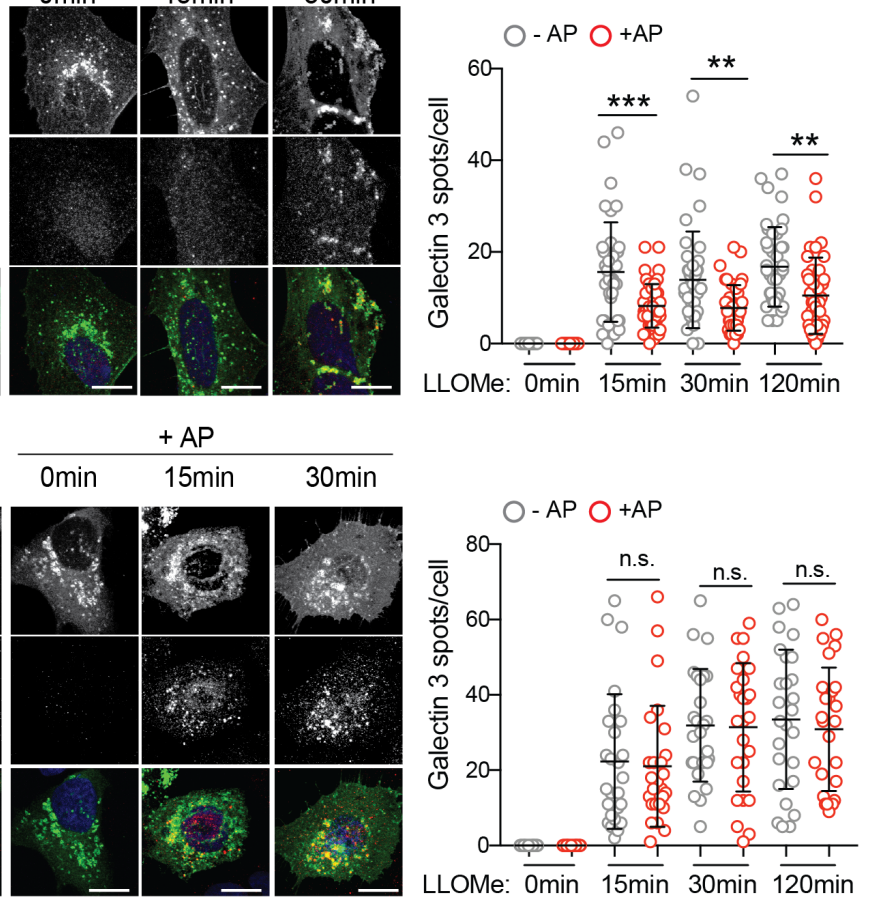

Supplementary Figure 5 | Lysosomal targeting of MYOF delays onset of membrane damage.

738 a. U20S cells stably expressing T192-Flag-FKBP and transiently transfected with MYOF-FRB* were treated with $1 \mathrm{mM}$ LLOMe for the indicated time points in the presence or absence of AP, followed by immuno-staining for HA (green) and Galectin 3 (GAL3; red). Recruitment of MYOF-

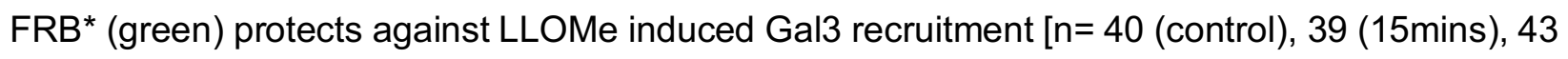

742 (30 mins), 41 (120mins) cells in the absence of AP and $\mathrm{n}=39$ (control), 45 (15mins), 41 (30mins) 743 and 39 (120mins) cells in the presence of AP]. b. U20S cells stably expressing T192-Flag-FKBP

744 and transfected with MYOF $\Delta$ C2-FRB* variant were treated as in 'a', followed by immuno-staining 745 for HA (green) and ALIX (red). Recruitment of MYOF $\triangle \mathrm{C2}$ (green) does not protect against LLOMe 746 induced ALIX recruitment [ $=26$ cells per condition (-AP and +AP)]. Graphs at right show 747 quantification of GAL3 (top) and ALIX (bottom) spots per cell in response to LLOMe. Scale, 20 $\mu \mathrm{m}$ 748 for all panels. Data are mean \pm s.d. $P$ values determined by unpaired two-tailed $t$-tests. ${ }^{* *} P<$ $7490.01 ;{ }^{* * *} P<0.001 ;$ n.s. not significant. 
a

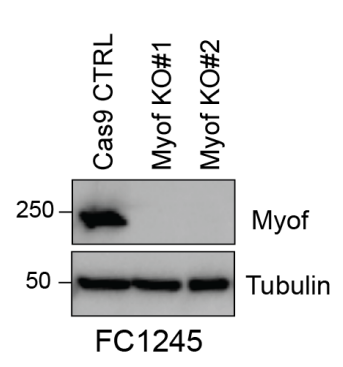

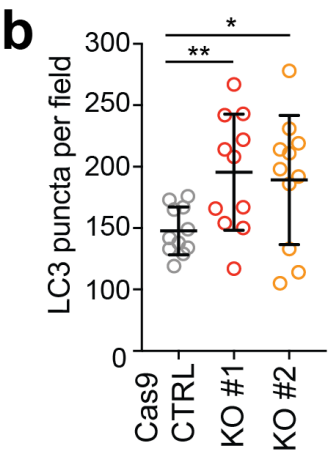
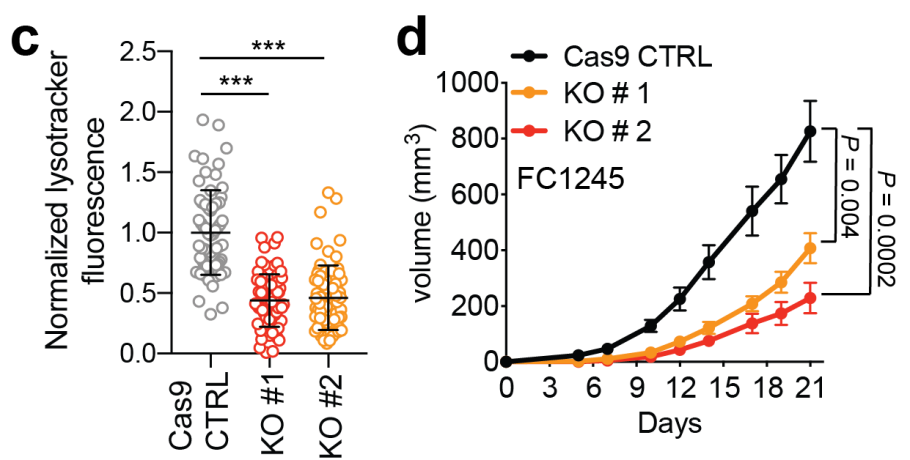

\section{Supplementary Figure 6 | MYOF is required for PDA tumour growth.}

753 a. Immunoblot of the indicated proteins in mouse KPC cells (FC1245) following CRISPR mediated

754 knockout of Myof. b. Graph showing increased accumulation of LC3B positive autophagosomes

755 in FC1245 Myof KO cells quantified from $\mathrm{n}=11$ fields per condition. Data are mean \pm s.d. c.

756 Graph showing decrease in lysotracker red staining in FC1245 Myof KO cells compared to Cas9

757 control cells, quantified from $n=70$ (Cas9 control), 71 (KO\#1), 70 (KO\#2) cells per condition.

758 Data are mean \pm s.d. d. Growth rate of Cas9 control and Myof KO FC1245 allografts following

759 s.c. transplantation in syngeneic C57BL/6 host mice ( $n=5-6$ animals per group). Data represent

760 mean \pm s.e.m. $P$ values determined by unpaired two-tailed $t$-tests. ${ }^{*} P<0.05 ;{ }^{* *} P<0.01 ;{ }^{* *} P$ $761<0.001$. 


\section{Methods}

Cell culture and reagents.

The cell lines HPDE, PaTu-8988T, KP4, MiaPaca2, Panc 2.03, PaTu-8902, Panc1, AsPc1, HPAFII, YAPC, Panc 3.27, Panc 10.05, Capan 2 and HupT3 were obtained from the American Type Culture Collection (ATCC) or the DSMZ. Cells were cultured in the following media: PaTu-8988T, KP4, MiaPaca2, PaTu-8902, Panc1, AsPc1, HPAFII, YAPC, Capan2, HEK293T in DMEM supplemented with 10\% FBS; Panc 2.03, Panc 3.27, Panc 10.05 and HupT3 in RPMI with 10\% FBS; HPDE cells were cultured in keratinocyte serum-free (KSF) medium supplemented by epidermal growth factor and bovine pituitary extract (Life Technologies, Inc., Grand Island, NY). Cell lines were regularly tested and verified to be mycoplasma negative using MycoAlert Detection Kit (Lonza).

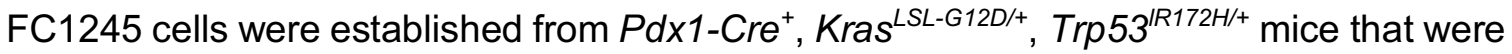
backcrossed into a C57BL/6 background and were a gift from David Tuveson (CSHL). Murine PDA cells were maintained in DMEM (Corning) supplemented with 10\% FBS (Atlanta Biologicals $\mathrm{S} 11550 \mathrm{H}$ ) and 1\% Pen/Strep (Gibco). Cells were grown in a humidified incubator with 5\% CO2 at $37^{\circ} \mathrm{C}$. Cultures were routinely verified to be negative for mycoplasma by PCR. Cell lines were authenticated by fingerprinting, and low passage cultures were carefully maintained in a central lab cell bank. were prepared in dimethyl sulfoxide (DMSO) and stored at $-80^{\circ} \mathrm{C}$ in single-use aliquots. Silica nanoparticles (cat.no. tIrl-sio; InvivoGen, San Diego, CA, USA) were suspended in ultrapure water according to manufactures instructions and diluted in complete growth medium to $100 \mu \mathrm{g} / \mathrm{ml}$. For hypertonic shock, $0.5 \mathrm{M}$ sucrose was made in complete growth medium and cells were incubated for the indicated time points. Lysotracker Red-DND-99 (L7528) was purchased from Thermo scientific and used at 75nM. DQ-BSA purchased from Thermo scientific (D12050).

\section{Constructs.}

790 pcDNA3.1-Myoferlin-HA and peGFP-hGalectin-3 was purchased from Addgene (plasmid no. 79122443 and 73080 respectively). T192-RFP-3xHA - was generated by subcloning the cDNA of

792 TMEM192 (Origene) together with monomeric Red Fluorescent Protein (mRFP) and 3xHA tag 793 into the Nhe1 and EcoR1 sites of pLJM1 lentiviral vector (addgene plasmid no. 134631) as

794 previously described $^{9}$. To generate MYOF $\triangle$ TM-HA-FRB*, first an Agel cut-site was knocked into 795 the pcDNA3.1-Myoferlin-HA plasmid immediately prior to the transmembrane domain by site- 
796 directed mutagenesis (Agilent: 210518). Second, a gBlock (IDT) containing HA fused to FRB*

797 was subcloned into the Agel and Kpnl sites of MYOF-HA (Addgene plasmid, \#22443) to generate

798 MYOF $\triangle$ TM-HA-FRB* (referred to as MYOF-FRB*). MYOF $\triangle$ C2 was generated via PCR

799 amplification from the MYOF-FRB* parent vector. The T192-FLAG-FKBP was a gift from Roberto

800 Zoncu at UC Berkeley.

801

802 shRNAs and siRNAs

803 shRNA vectors (pLKO.1 puro) were obtained from the Sigma MISSION TRC shRNA library. The

804 sequences and RNAi Consortium clone IDs for the shRNAs used are as follows: shMYOF\#1

805 (human): 5'- GAAAGAGCTGTGCATTATAAA-3' (TRCN0000320397); shMYOF\#2 (human): 5'-

806 GAAAGAGCTGTGCATTATAAA-3' (TRCN0000001522); $\quad$ shATG3\#1 (human): 5'-

807 GATGTGACCATTGACCATATT-3' (TRCN0000148120); $\quad$ shATG3\#2 (human): 5'-

808 GCTGTCATTCCAACAATAGAA-3' (TRCN0000147381); $\quad$ shATG7\#1 (human): 5'-

809 CCCAGCTATTGGAACACTGTA-3' (TRCN0000007587); $\quad$ shATG7\#2 (human): 5'-

810 GCCTGCTGAGGAGCTCTCCAT-3' (TRCN0000007584); shGFP: 5'-

811 TGCCCGACAACCACTACCTGA-3' (TRCN0000072186). Pre-designed silencer select siRNAs

812 against MYOF were ordered from Thermo Fischer (Cat\# 4392420). shGFP and shScr (Addgene,

813 plasmid \#17920) were used as controls.

\section{Lentiviral-mediated knockdown}

816 For the transfection of lentiviral vectors (pSLIK-hygro, pINDUCER20, and pLKO.1-puro), lentivirus

817 was produced by co-transfection of HEK293T cells with a lentiviral vector and the packaging 818 plasmids psPAX2 (Addgene, plasmid \#12260) and pMD2.G (Addgene, plasmid \#12259) at a 819 0.5:0.25:0.25 ratio. Transfection was performed using X-tremeGENE transfection (6365787001;

820 Sigma Aldrich) reagent according to the manufacturer's instructions. The viral supernatant was 821 collected $48 \mathrm{~h}$ after transfection and filtered through a $0.45 \mu \mathrm{m}$ filter. Cells were infected with virus822 containing media using Polybrene reagent (TR-1003-G; EMD Millipore) according to the 823 manufacturer's instructions and selected for $48 \mathrm{hr}$ in $2 \mu \mathrm{g} / \mathrm{mL}$ of puromycin.

\section{Immunoblotting}

826 Cells were lysed in ice-cold lysis buffer $(150 \mathrm{mM} \mathrm{NaCl}, 20 \mathrm{mM}$ Tris [pH 7.5], $1 \mathrm{mM}$ EDTA, $1 \mathrm{mM}$

827 EGTA, 1\% Triton X-100, $2.5 \mathrm{mM}$ sodium pryophosphate, $1 \mathrm{mM} \beta$-glycerophosphate, $1 \mathrm{mM}$ 828 sodium vanadate, and one tablet of Pierce Protease Inhibitor Tablets, EDTA Free [Fisher 829 Scientific-A32965] per $10 \mathrm{~mL}$ ). Protein content was measured using Pierce BCA Protein Assay 
830 Kit (Life Technologies-23227), and 20-30 $\mu$ g protein was resolved on 8, 10 or $12 \%$ protein gels 831 using SDS-PAGE and transferred onto PVDF membranes (EMD MIllipore-IPVH00010).

832 Membranes were blocked in 5\% bovine serum albumin (BSA, Sigma Aldrich-A4503) made up in

833 Tris-buffered saline with $0.2 \%$ Tween 20 (TBS-T) prior to incubation with primary antibody

834 overnight at $4^{\circ} \mathrm{C}$ in $5 \%$ bovine serum albumin. Membranes were washed in TBS-T and developed

835 after $1 \mathrm{~h}$ incubation in species-specific horseradish peroxidase-conjugated secondary antibody,

836 visualized using supersignal west pico chemiluminescent substrate (Fisher Scientific-34080), and

837 imaged using the ChemiDoc XRS + System (Biorad).

\section{Immunoprecipitation}

840 For lysosome immunoprecipitation experiments, human PDA, HPDE or HEK293T cell lines stably 841 expressing T192-mRFP-3xHA were lysed and intact lysosomes from 1-2 mg of total protein was 842 immunoprecipitated using anti-HA-conjugated Dynabeads (Thermo Sci. 88837) as previously 843 described ${ }^{1,2}$. Proteinase K (Sigma P.2308) digestion was performed when lysosomes were bound 844 to anti-HA-beads. Increasing concentrations of Proteinase $\mathrm{K}(0.1,0.5,1,2.5 \mathrm{ug} / \mathrm{ml})$ in Proteinase $845 \mathrm{~K}$ buffer (33.3mM Hepes, $1 \mathrm{mM} \mathrm{CaCl}$, $\mathrm{pH}: 7.4$ ) was added to lysosomes bound to anti-HA beads 846 for $15 \mathrm{~min}$ on ice. Digestion was terminated with $2 \mathrm{mM}$ phenylmethylsulfonyl fluoride (final concentration). Lysosomal proteins were separated by SDS-PAGE and proteins were detected by western blotting.

\section{Surface Biotinylation assay}

851 The cell surface of KP4 cells and MYOF-HA expressing HEK293T cells was biotinylated on ice 852 using $0.5 \mathrm{mg} / \mathrm{ml}$ of EZ-Link ${ }^{\mathrm{TM}}$ Sulfo-NHS-SS-Biotin (Thermo Scientific: 21331) for 30min. Cells 853 were washed twice with $20 \mathrm{mM}$ Glycine in HBSS on ice to remove excess biotin. Subsequently, 854 cells were lysed in Biotinylation Lysis Buffer (1\% Triton X-100, 130mM NaCl, 2.5mM MgCl $2,2 \mathrm{mM}^{2}$ 855 EGTA, 25mM HEPES, pH 7.4, supplemented with protease inhibitor prior to use) on ice for 30min. 856 Lysates were clarified by centrifugation at $13,300 \mathrm{rpm}$ for $10 \mathrm{~min}$ at $4^{\circ} \mathrm{C}$ and supernatants were 857 transferred to a new tube. Lysates were quantified by Pierce BCA Protein Assay Kit and equal 858 amounts $(\sim 1 \mathrm{mg})$ of protein were incubated with $100 \mu$ l of triple-washed Dynabeads ${ }^{\mathrm{TM}} \mathrm{MyOne}^{\mathrm{TM}}$ 859 Streptavidin C1 beads (Invitrogen: 65002) for 2-3h with constant agitation. Beads and captured 860 materials were washed twice in Wash Buffer 1 (2\% SDS in $\mathrm{dH} 2 \mathrm{O})$, once in Wash Buffer $2(0.1 \%$ 861 deoxycholate, $1 \%$ Triton X-100, 500mM NaCl, $1 \mathrm{mM}$ EDTA, and 50mM HEPES, pH 7.5), once in 862 Wash Buffer 3 (250mM LiCl, 0.5\% NP-40, 0.5\% deoxycholate, 1mM EDTA, and 10mM Tris, $\mathrm{pH}$ 
863 8.1), and twice in Wash Buffer 4 (50mM Tris, $\mathrm{pH} 7.4$, and 50mM NaCl). Washes were performed

864 at RT for 5 min with gentle agitation. Samples were eluted by boiling in Laemlli buffer.

FRB-FKBP heterodimerization assay.

867 U2OS cells stably expressing T192-Flag-FKBP were grown on coverslips and transiently 868 transfected with either MYOF-FRB* or MYOF $\triangle \mathrm{C} 2-\mathrm{FRB}^{*}$ using X-tremegene 9 DNA Transfection

869 Reagent (Roche). After 24h, dimerization was induced by adding 100nM of AP21967 (Takara:

870 635056) for $1 \mathrm{~h}$ to cells. Coverslips were then washed twice prior to treated with $1 \mathrm{mM}$ LLOME for

871 the indicated timepoints. Cells were subsequently fixed for immunofluorescent staining.

\section{Immunofluorescence}

874 Human cells were cultured for two days on coverslips coated with fibronectin. After two PBS 875 washes, cells were fixed with paraformaldehyde for $15 \mathrm{~min}$ at room temperature or with ice-cold 876 methanol for $5 \mathrm{~min}$ at $-20^{\circ} \mathrm{C}$. PFA fixed cells were permeabilized with $0.1 \%$ Saponin or $0.3 \%$ Triton $877 \mathrm{X}-100$ for $5 \mathrm{~min}$. Samples were then blocked with $5 \%$ normal goat serum for $15 \mathrm{~min}$ at room 878 temperature prior to incubation with primary antibodies overnight at $4^{\circ} \mathrm{C}$ (Supplementary Table 879 1). After washing three times with PBS, cells were incubated in secondary antibody (diluted 1:400 880 in PBS) at room temperature for $30 \mathrm{~min}$. Slides were mounted on glass slides using DAPI 881 Fluoromount-G (0100-20, SouthernBiotech) and imaged on a Zeiss Laser Scanning Microscope 882 (LSM) 710 using a 63x objective. Image processing and quantification were performed using

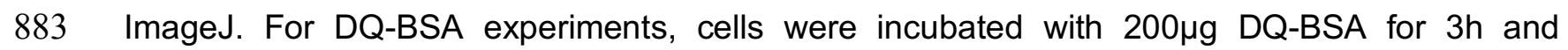
884 subsequently chased for $3 \mathrm{~h}$ in DQ-BSA free media. Where indicated, treatment with BafA1 was 885 for $1 \mathrm{~h}$ at $100 \mathrm{nM}$. The number of DQ-BSA spots co-localizing with LAMP2 positive lysosomes was 886 quantified. For experiments requiring exposure to LLOMe, silica nanoparticles, hypertonic 887 sucrose or Lysotracker, coverslips were bathed in medium containing the appropriate reagent as 888 indicated, prior to fixing and staining. Measurement of colocalization was conducted using 889 thresholded images using the image calculator function in Image J. Overlapping pixels in the red 890 and green channels were measured and percentage overlap calculated from a minimal of 10 891 fields per condition. For measuring total cell fluorescence, analysis was performed on a per cell 892 basis using Image J. Mean fluorescence intensities for each cell was determined by subtracting 893 the mean fluorescence of the background in each image. 
896 Myoferlin knockouts in KP4, Patu8902 and FC1245 cells were generated using the RNP-

897 electroporation method as previously described (Liang et al., 2015). One million cells were

898 electroporated using the Amaxa 4D Nucleofector kit (V4XC-9064, Lonza). Guide RNA and Cas9

899 complexes were pre-formed using 160 $\mu \mathrm{M}$ crRNA annealed to $160 \mu \mathrm{M}$ tracrRNA (Dharmacon) and

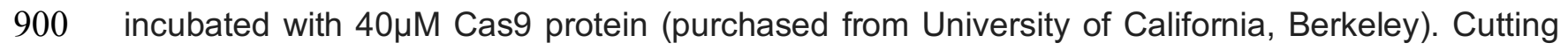

901 efficiency was assessed 48h post-electroporation using PCR and sanger sequencing. Myoferlin

902 knockout was confirmed using quantitative RT-PCR and immunoblotting after clonal expansion

903 of single cells.

904

905 Guide RNA Sequences (5'-3'):

906 Mouse Myoferlin exon 3 - TGACTT GAG GGG GAT ACC AC

907 Mouse Myoferlin exon 4 - CTC CCT GAA GGA CCT GAT TG

908 Human MYOFERLIN exon 1 - TTT CGT TTT AGG GAT ATT GC

909 Human MYOFERLIN exon 3 - ATT TTG GAG TTT GAC TTG AG

910

911 PCR Primer Sequences (5'-3'):

912 Ms Myof Ex3 Fwd - tgagtcagaggtttggtgaccc

913 Ms Myof Ex3 Rev - cagactcgtgacggctgagtat

914 Ms Myof Ex4 Fwd - agccaaagagaggagcatgtgt

915 Ms Myof Ex4 Rev - tatctcaacctcccaactgccg

916 Hu MYOF Ex1 Fwd - GGGAGTTCGGTATCAGTTTACA

917 Hu MYOF Ex1 Rev - CTGGAGAGACTTGGCTTCATC

918 Hu MYOF Ex 3 Fwd - TCAGCTGCCTTCAGGTTTAG

919 Hu MYOF Ex3 Rev - CCACATCTGCTATTGGCTTAGA

\section{Histology and immunostaining}

922 Tissue samples were fixed overnight in 10\% formalin, and then embedded in paraffin and

923 sectioned (5mm thickness) by the UCSF mouse histopathology core. Haematoxylin and eosin

924 staining was performed using standard methods. Slides were baked at $60^{\circ} \mathrm{C}$ for a $1 \mathrm{~h}$,

925 deparaffinized in xylenes (three treatments, 5 min each), rehydrated sequentially in ethanol (5min

926 in $100 \%, 5 \mathrm{~min}$ in $90 \%, 5 \mathrm{~min}$ in $70 \%, 5 \mathrm{~min}$ in $50 \%$, and $5 \mathrm{~min}$ in $30 \%$ ), and washed for $5 \mathrm{~min}$ in

927 water twice. For antigen unmasking, specimens were cooked in a $10 \mathrm{mM}$ sodium citrate buffer $(\mathrm{pH}$

928 6.0) for $10 \mathrm{~min}$ at $95^{\circ} \mathrm{C}$ using conventional pressure cooker, rinsed three times with PBS,

929 incubated for $1 \mathrm{~h}$ with $3 \% \mathrm{H}_{2} \mathrm{O}_{2}$ at room temperature to block endogenous peroxidase activity, 
930 washed three times with PBS, and blocked with $2.5 \%$ goat serum in PBS for $1 \mathrm{~h}$. Primary

931 antibodies were diluted in blocking solution and incubated with the tissue sections at $4^{\circ} \mathrm{C}$

932 overnight. Specimens were then washed three times for $5 \mathrm{~min}$ each in PBS and incubated with

933 secondary anti-mouse/rabbit IgG (Vector Laboratories, MP-7500) or fluorescent-conjugated

934 secondary antibodies (1:500, Supplementary Table 1) at RT for $1 \mathrm{~h}$. Following three washes in

935 PBS, slides were stained for peroxidase for 3 min with the DAB (di-aminebenzidine) substrate kit

936 (SK-4100, Vector Laboratories), washed with water and counterstained with haematoxylin or

937 mounted on glass slides using DAPI Fluoromount-G. Bright light images were obtained with a

938 with a KEYENCE BZ-X710 microscope. Fluorescent images were obtained with Zeiss Laser

939 Scanning Microsope (LSM) 710.

941 Human Samples

942 The pancreatic cancer tissue microarray has been previously described ${ }^{3}$. Clinicopathologic 943 variables used for analysis of the TMA were based on the $7^{\text {th }}$ edition of the AJCC/UICC TNM 944 staging system for pancreatic cancer. Immunohistochemical staining for MYOF was conducted 945 using anti-MYOF ab (Sigma; Cat. No HPA014245). Multiple (2-3) $1.0 \mathrm{~mm}$ cores for each tumour 946 in the TMA were independently scored for staining positivity by two pathologists (DWD and KWW) 947 using modified histoscores (range 0-9), representing the product of staining intensity (0-3, 0948 absent; 1-weak; 2-moderate; 3-strong) and percentage of tumour cell staining $(0-3,0$, none; 1, 1$94933 \% ; 2,34-66 \% ; 3,67-100 \%$ ). For any core where histoscore differed by more than 2 between 950 the two observers, a revised score was assigned based on consensus evaluation. The average 951 histoscore for both observers was used for subsequent analysis. For dichotomization, each 952 tumour was assigned to either a low (histoscore 0-5) or high (histoscore >5-9) staining group. 953 Survival estimates were generated using the Kaplan-Meier method and compared using log-rank 954 tests. Multivariate Cox proportional hazards models were used to test statistical independence 955 and significance of multiple predictors with backward selection performed using the Akaike 956 Information Criterion. Overall survival time was measured from the date of surgery to the date of 957 death due to any cause or last clinical visit up to 60 months.

958 PDAC expression data was collected from the following databases: GSE62452, GSE28735, 959 GSE15471, GSE16515, GSE43795. $P$ values were calculated by paired or unpaired, two-tailed $t$ 960 test.

961

962 Animal Experiments 
All experiments were carried out in a clean conventional facility. For subcutaneous xenografts, 2 million KP4 Cas9 control/MYOF KO or FC1245 Cas9 control/myof KO cells were injected into the flank of nude mice and C57BI/6 mice respectively (5-6 mice per group). Tumour length and width were measured thrice a week and the volume was calculated according to the formula (length $\mathrm{x}$ width $\left.^{2}\right) / 2$. Mice were euthanized when tumour volume reached $800-1000 \mathrm{~mm}^{3}$ and tumours were

968 harvested and submitted for histological examination. Mouse strains were obtained from Jackson

969 Laboratories and kindly provided by colleagues: Ptf1a-Cre (Jax stock no. 019378) mice from C.

970 Wright, LSL-Kras ${ }^{\text {G12D }}$ (Jax stock no. 008179) mice from D. Tuveson and T. Jacks and Tp53 ${ }^{\text {Lox/Lox }}$

971 (Jax stock no. 008462) mice from A. Berns.

972

\section{Transmission electron microscopy}

974 KP4 cells (control and Myoferlin knockdown cells) grown on tissue culture plates were fixed 975 in $2.5 \%$ glutaraldehyde $0.1 \mathrm{M}$ sodium cacodylate buffer, pH 7.4 (EMS, Hatfield, PA, USA) for $97630 \mathrm{~min}$ at room temperature and then gently scraped using a rubber policeman. Cell pellets were 977 stabilized in $1 \%$ low melting point agarose, then cut into $1 \mathrm{~mm}$ cubes. Samples were rinsed (3x;

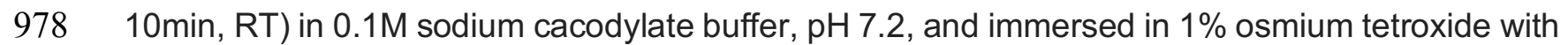
$9791.6 \%$ potassium ferricyanide in $0.1 \mathrm{M}$ sodium cacodylate buffer for 1 hour. Samples were rinsed $980(3 \mathrm{x} ; 10 \mathrm{~min}, \mathrm{RT})$ in buffer and then in distilled water $(3 \mathrm{x} ; 10 \mathrm{~min}, \mathrm{RT})$. Sample were immersed in $9810.5 \%$ aqueous uranyl acetate en bloc stain for $1 \mathrm{~h}$, protected from light and then rinsed in water $982(3 \mathrm{x} ; 10 \mathrm{~min}, \mathrm{RT})$. Samples were then subjected to an ascending acetone gradient $(10 \mathrm{~min} ; 35 \%$, $98350 \%, 70 \%, 80 \%, 90 \%)$ followed by pure acetone (2x; 10min, RT). Samples were progressively 984 infiltrated while rocking with Epon resin (EMS, Hatfield, PA, USA) and polymerized at $60{ }^{\circ} \mathrm{C}$ for 985 24-48h. Thin sections (70nm) were cut using a Reichert Ultracut E (Leica, Wetzlar, Germany). 986 Sections were then collected onto formvar-coated 200 mesh copper grids. The grids were post987 stained with $2 \%$ uranyl acetate followed by Reynold's lead citrate, for $5 \mathrm{~min}$ each. The sections 988 were imaged using a Tecnai 12 120kV TEM (FEI, Hillsboro, OR, USA) and data recorded using 989 an UltraScan 1000 with Digital Micrograph 3 software (Gatan Inc., Pleasanton, CA, USA).

\section{Pathway analysis and statistical analysis}

992 A list of $\geq 2$ fold significantly enriched ( $p$-value $\leq 0.05$; $\log _{2}$ fold change $\geq 1$ ) proteins present in 993 PDA lysosome elutes was analyzed for significantly overrepresented gene ontology (GO) terms 994 (Biological Processes) and biological pathways (KEGG) using g:Profiler g:GO St tool ${ }^{4}$ and The 995 Database for Annotation, Visualization and Integrated Discovery (DAVID). 
996 Experimental data were analyzed using GraphPad Prism and excel built-in tests and are

997 indicated in the figure legends. For all graphs, error bars indicate mean \pm standard deviation

998 unless otherwise indicated. Numbers of samples analyzed per experiment are reported in the

999 respective figure legends.

1000

1001

1002 References for methods

10031 Zoncu, R. et al. mTORC1 senses lysosomal amino acids through an inside-out mechanism

1004 that requires the vacuolar $\mathrm{H}(+)$-ATPase. Science 334, 678-683,

1005 doi:10.1126/science.1207056 (2011).

10062 Abu-Remaileh, M. et al. Lysosomal metabolomics reveals V-ATPase- and mTOR-

1007 dependent regulation of amino acid efflux from lysosomes. Science 358, 807-813, 1008 doi:10.1126/science.aan6298 (2017).

10093 Manuyakorn, A. et al. Cellular histone modification patterns predict prognosis and

1010 treatment response in resectable pancreatic adenocarcinoma: results from RTOG 9704. $J$

1011 Clin Oncol 28, 1358-1365, doi:10.1200/JCO.2009.24.5639 (2010)

10124 Raudvere, U. et al. g:Profiler: a web server for functional enrichment analysis and

1013 conversions of gene lists. Nucleic Acids Res 47, W191-W198, doi:10.1093/nar/gkz369

1014 (2019). 
bioRxiv preprint doi: https://doi.org/10.1101/2021.01.04.425106; this version posted January 4, 2021. The copyright holder for this preprint

(which was not certified by peer review) is the author/funder, who has granted bioRxiv a license to display the preprint in perpetuity. It is made available under aCC-BY-NC-ND 4.0 International license.

\section{Supplementary Tables}

1016

1017 Table 1: $\geq 2$ fold significantly enriched proteins identified in PDA lysosome elutes.

1018 Table 2: Cohort of $\geq 2$ fold significantly enriched PDA lysosomal proteins associated with vesicle 1019 trafficking and endocytosis.

1020 Table 3: Clinicopathological characteristics and group membership in UCLA TMA.

1021 Table 4: Cox proportional hazard models for prognostic factors. 
bioRxiv preprint doi: https://doi.org/10.1101/2021.01.04.425106; this version posted January 4, 2021. The copyright holder for this preprint (which was not certified by peer review) is the author/funder, who has granted bioRxiv a license to display the preprint in perpetuity. It is made available under aCC-BY-NC-ND 4.0 International license.

\section{Supplementary Table 1: >2 fold significantly enriched proteins identified in PDA} lysosome elutes

\begin{tabular}{|c|c|c|c|c|c|c|}
\hline Accession Number & $\begin{array}{c}\text { avg } \\
\text { HEK293T }\end{array}$ & $\begin{array}{c}\text { avg } \\
\text { PaTu8988T }\end{array}$ & $\begin{array}{c}\text { fold } \\
\text { (PaTu8988T/ } \\
\text { HEK293T) }\end{array}$ & ttest & $\begin{array}{l}\text { log2 } \\
\text { fold } \\
\text { change }\end{array}$ & $\begin{array}{c}(-\log 10 \\
\text { ttest })\end{array}$ \\
\hline NP_001123927 & 1 & 89.66667 & 89.66667 & 7.1E-05 & 6.486 & 4.146 \\
\hline NP_002517 & 1 & 68.66667 & 68.66667 & $6.5 \mathrm{E}-06$ & 6.102 & 5.188 \\
\hline NP_002195 & 1 & 52.33333 & 52.33333 & 1E-05 & 5.71 & 4.993 \\
\hline NP_079105 & 1 & 50.66667 & 50.66667 & $1.2 \mathrm{E}-05$ & 5.663 & 4.936 \\
\hline XP_005264575 & 0.66667 & 23.66667 & 35.5 & 0.00014 & 5.15 & 3.868 \\
\hline XP_005264575 & 0.66667 & 23.66667 & 35.5 & 0.00014 & 5.15 & 3.868 \\
\hline NP_002107 & 1 & 34.66667 & 34.66667 & 0.00025 & 5.115 & 3.606 \\
\hline NP_001290453 & 1 & 33.66667 & 33.66667 & 8.7E-05 & 5.073 & 4.061 \\
\hline NP_001093640 & 1 & 32 & 32 & $1.1 \mathrm{E}-05$ & 5 & 4.941 \\
\hline NP_001193773 & 1 & 31.66667 & 31.66667 & 0.00096 & 4.985 & 3.016 \\
\hline XP_016856023 & 1 & 31.33333 & 31.33333 & $3.1 \mathrm{E}-05$ & 4.97 & 4.507 \\
\hline XP_011543028 & 1 & 31 & 31 & 0.00013 & 4.954 & 3.871 \\
\hline NP_038479 & 3.66667 & 108 & 29.45455 & 3E-07 & 4.88 & 6.52 \\
\hline NP_038479 & 3.66667 & 108 & 29.45455 & $3 \mathrm{E}-07$ & 4.88 & 6.52 \\
\hline NP_001073286 & 1 & 27.66667 & 27.66667 & $5.2 \mathrm{E}-05$ & 4.79 & 4.285 \\
\hline NP_000201 & 1 & 27 & 27 & 2.3E-05 & 4.755 & 4.638 \\
\hline XP_006711978 & 1 & 26.66667 & 26.66667 & 0.00039 & 4.737 & 3.411 \\
\hline XP_016874073 & 1 & 26.33333 & 26.33333 & 4.5E-05 & 4.719 & 4.345 \\
\hline XP_016874073 & 1 & 26.33333 & 26.33333 & 4.5E-05 & 4.719 & 4.345 \\
\hline NP_001073286 & 1 & 26.33333 & 26.33333 & 0.00014 & 4.719 & 3.865 \\
\hline XP_005266132 & 1 & 26 & 26 & 0.00041 & 4.7 & 3.384 \\
\hline NP_004159 & 1 & 25 & 25 & 0.0024 & 4.644 & 2.62 \\
\hline NP_004159 & 1 & 25 & 25 & 0.0024 & 4.644 & 2.62 \\
\hline XP_011514006 & 1 & 24 & 24 & 0.00134 & 4.585 & 2.871 \\
\hline NP_001007069 & 1 & 23.33333 & 23.33333 & 4.7E-06 & 4.544 & 5.325 \\
\hline NP_000253 & 1 & 22.66667 & 22.66667 & $1.6 \mathrm{E}-05$ & 4.503 & 4.788 \\
\hline NP_009220 & 1 & 22.33333 & 22.33333 & 3.6E-07 & 4.481 & 6.447 \\
\hline NP_001007069 & 1 & 22.33333 & 22.33333 & 5.7E-06 & 4.481 & 5.245 \\
\hline NP_000593 & 1 & 21.66667 & 21.66667 & $2 \mathrm{E}-05$ & 4.437 & 4.706 \\
\hline NP_001229971 & 1 & 21.33333 & 21.33333 & 0.00236 & 4.415 & 2.627 \\
\hline NP_001007070 & 1 & 21 & 21 & 4.1E-06 & 4.392 & 5.383 \\
\hline NP_001035861 & 1 & 20.66667 & 20.66667 & 0.00037 & 4.369 & 3.434 \\
\hline XP_016883302 & 1 & 18.33333 & 18.33333 & 0.00013 & 4.196 & 3.872 \\
\hline XP_011531200 & 1 & 17.66667 & 17.66667 & 4.6E-05 & 4.143 & 4.336 \\
\hline NP_001157789 & 0.66667 & 11 & 16.5 & 0.00344 & 4.044 & 2.463 \\
\hline NP_001257904 & 0.66667 & 10.66667 & 16 & 0.0019 & 4 & 2.722 \\
\hline NP_005403 & 0.66667 & 10.33333 & 15.5 & 0.00292 & 3.954 & 2.535 \\
\hline
\end{tabular}


bioRxiv preprint doi: https://doi.org/10.1101/2021.01.04.425106; this version posted January 4, 2021. The copyright holder for this preprint (which was not certified by peer review) is the author/funder, who has granted bioRxiv a license to display the preprint in perpetuity. It is made available under aCC-BY-NC-ND 4.0 International license.

\begin{tabular}{|c|c|c|c|c|c|c|}
\hline NP_005403 & 0.66667 & 10.33333 & 15.5 & 0.00292 & 3.954 & 2.535 \\
\hline NP_037364 & 1 & 15.33333 & 15.33333 & 0.00356 & 3.939 & 2.448 \\
\hline NP_005219 & 4.66667 & 70.33333 & 15.07143 & 0.00012 & 3.914 & 3.922 \\
\hline NP_005219 & 4.66667 & 70.33333 & 15.07143 & 0.00012 & 3.914 & 3.922 \\
\hline NP_443164 & 1 & 14.66667 & 14.66667 & 0.00149 & 3.874 & 2.825 \\
\hline NP_057231 & 0.66667 & 9.666667 & 14.5 & 0.00067 & 3.858 & 3.172 \\
\hline NP_001679 & 0.66667 & 9.666667 & 14.5 & 0.00882 & 3.858 & 2.054 \\
\hline NP_065843 & 1 & 14.33333 & 14.33333 & 0.00038 & 3.841 & 3.425 \\
\hline NP_001247419 & 1 & 13.66667 & 13.66667 & 0.00199 & 3.773 & 2.701 \\
\hline NP_000916 & 1 & 13.66667 & 13.66667 & 0.00199 & 3.773 & 2.701 \\
\hline$X P \_016856023$ & 2.66667 & 35 & 13.125 & $2.4 \mathrm{E}-05$ & 3.714 & 4.617 \\
\hline NP_001611 & 8.66667 & 112 & 12.92308 & 0.00141 & 3.692 & 2.851 \\
\hline NP_001611 & 8.66667 & 112 & 12.92308 & 0.00141 & 3.692 & 2.851 \\
\hline NP_003557 & 2.33333 & 29.66667 & 12.71429 & 0.00057 & 3.668 & 3.241 \\
\hline NP_060530 & 1 & 12.66667 & 12.66667 & $4 \mathrm{E}-06$ & 3.663 & 5.4 \\
\hline NP_001020527 & 1 & 12.66667 & 12.66667 & 0.00063 & 3.663 & 3.2 \\
\hline NP_001020527 & 1 & 12.66667 & 12.66667 & 0.00063 & 3.663 & 3.2 \\
\hline NP_004915 & 5.66667 & 71 & 12.52941 & $3.2 \mathrm{E}-06$ & 3.647 & 5.499 \\
\hline XP_011528984 & 1.33333 & 16.33333 & 12.25 & 0.00279 & 3.615 & 2.555 \\
\hline NP_003253 & 0.66667 & 8 & 12 & 0.00114 & 3.585 & 2.942 \\
\hline NP_006283 & 1 & 12 & 12 & 0.00197 & 3.585 & 2.705 \\
\hline NP_001171114 & 0.66667 & 8 & 12 & 0.01169 & 3.585 & 1.932 \\
\hline NP_000518 & 1.33333 & 15.66667 & 11.75 & 0.00065 & 3.555 & 3.184 \\
\hline NP_004915 & 4.66667 & 53.33333 & 11.42857 & $1.9 \mathrm{E}-05$ & 3.515 & 4.725 \\
\hline NP_001690 & 1 & 11.33333 & 11.33333 & 0.00101 & 3.503 & 2.998 \\
\hline NP_000174 & 1 & 11.33333 & 11.33333 & 0.00272 & 3.503 & 2.566 \\
\hline NP_000174 & 1 & 11.33333 & 11.33333 & 0.00272 & 3.503 & 2.566 \\
\hline XP_006718556 & 1 & 11 & 11 & 0.00056 & 3.459 & 3.25 \\
\hline NP_079472 & 0.66667 & 7.333333 & 11 & 0.00086 & 3.459 & 3.063 \\
\hline NP_001017423 & 1.33333 & 14.33333 & 10.75 & 0.00124 & 3.426 & 2.905 \\
\hline NP_001093 & 3.66667 & 39 & 10.63636 & $2.9 \mathrm{E}-05$ & 3.411 & 4.534 \\
\hline NP_891988 & 4.33333 & 45.66667 & 10.53846 & $3.1 \mathrm{E}-06$ & 3.398 & 5.515 \\
\hline XP_006717309 & 2.66667 & 28 & 10.5 & $1.8 \mathrm{E}-05$ & 3.392 & 4.75 \\
\hline NP_003840 & 1.33333 & 14 & 10.5 & 0.00199 & 3.392 & 2.701 \\
\hline NP_570846 & 1 & 10.33333 & 10.33333 & 0.01001 & 3.369 & 2 \\
\hline NP_006491 & 1 & 10 & 10 & 9.9E-05 & 3.322 & 4.005 \\
\hline NP_006491 & 1 & 10 & 10 & $9.9 \mathrm{E}-05$ & 3.322 & 4.005 \\
\hline NP_079199 & 1.66667 & 16.33333 & 9.8 & 0.00204 & 3.293 & 2.691 \\
\hline NP_079199 & 1.66667 & 16.33333 & 9.8 & 0.00204 & 3.293 & 2.691 \\
\hline NP_000010 & 1.66667 & 16 & 9.6 & 0.00579 & 3.263 & 2.237 \\
\hline NP_006102 & 1 & 9.333333 & 9.333333 & 0.0007 & 3.222 & 3.155 \\
\hline NP_000681 & 1 & 9.333333 & 9.333333 & 0.00227 & 3.222 & 2.644 \\
\hline NP 001136336 & 0.66667 & 6 & 9 & $8.9 \mathrm{E}-05$ & 3.17 & 4.05 \\
\hline
\end{tabular}


bioRxiv preprint doi: https://doi.org/10.1101/2021.01.04.425106; this version posted January 4, 2021. The copyright holder for this preprint (which was not certified by peer review) is the author/funder, who has granted bioRxiv a license to display the preprint in perpetuity. It is made available under aCC-BY-NC-ND 4.0 International license.

\begin{tabular}{|c|c|c|c|c|c|c|}
\hline XP_011512751 & 1 & 9 & 9 & 0.00228 & 3.17 & 2.642 \\
\hline NP_003970 & 1 & 9 & 9 & 0.00228 & 3.17 & 2.642 \\
\hline NP_001289561 & 1 & 9 & 9 & 0.00635 & 3.17 & 2.197 \\
\hline NP_001289561 & 1 & 9 & 9 & 0.00635 & 3.17 & 2.197 \\
\hline NP_001034579 & 1 & 9 & 9 & 0.01613 & 3.17 & 1.792 \\
\hline NP_004484 & 0.66667 & 6 & 9 & 0.0329 & 3.17 & 1.483 \\
\hline NP_001449 & 4 & 35.33333 & 8.833333 & 0.00036 & 3.143 & 3.447 \\
\hline XP 005248242 & 1 & 8.666667 & 8.666667 & 0.00033 & 3.115 & 3.486 \\
\hline NP_000134 & 1 & 8.666667 & 8.666667 & 0.0031 & 3.115 & 2.509 \\
\hline NP_071436 & 1 & 8.333333 & 8.333333 & 0.00039 & 3.059 & 3.411 \\
\hline NP_002626 & 1 & 8.333333 & 8.333333 & 0.01417 & 3.059 & 1.849 \\
\hline NP_000204 & 1 & 8.333333 & 8.333333 & 0.0168 & 3.059 & 1.775 \\
\hline NP_000204 & 1 & 8.333333 & 8.333333 & 0.0168 & 3.059 & 1.775 \\
\hline NP_036475 & 1 & 8.333333 & 8.333333 & 0.02844 & 3.059 & 1.546 \\
\hline NP_000970 & 1.33333 & 11 & 8.25 & 0.00192 & 3.044 & 2.716 \\
\hline XP_016869431 & 4.33333 & 35.66667 & 8.230769 & 7.7E-05 & 3.041 & 4.114 \\
\hline NP_037506 & 4.66667 & 38.33333 & 8.214286 & 0.00518 & 3.038 & 2.286 \\
\hline NP_037506 & 4.66667 & 38.33333 & 8.214286 & 0.00518 & 3.038 & 2.286 \\
\hline NP_002831 & 2.66667 & 21.66667 & 8.125 & 2.3E-06 & 3.022 & 5.645 \\
\hline NP_009055 & 1 & 8 & 8 & 0.00027 & 3 & 3.576 \\
\hline NP_009055 & 1 & 8 & 8 & 0.00027 & 3 & 3.576 \\
\hline NP_955394 & 2 & 16 & 8 & 0.00374 & 3 & 2.427 \\
\hline NP_001309906 & 1 & 8 & 8 & 0.00374 & 3 & 2.427 \\
\hline NP_001309906 & 1 & 8 & 8 & 0.00374 & 3 & 2.427 \\
\hline NP_001186913 & 1 & 8 & 8 & 0.01016 & 3 & 1.993 \\
\hline NP_001688 & 1 & 8 & 8 & 0.0249 & 3 & 1.604 \\
\hline NP_955394 & 2 & 15.66667 & 7.833333 & 0.00378 & 2.97 & 2.423 \\
\hline NP_001001937 & 7.33333 & 57 & 7.772727 & 0.00071 & 2.958 & 3.151 \\
\hline NP_001310245 & 1 & 7.666667 & 7.666667 & 0.00056 & 2.939 & 3.25 \\
\hline NP_001310245 & 1 & 7.666667 & 7.666667 & 0.00056 & 2.939 & 3.25 \\
\hline NP_061329 & 1 & 7.666667 & 7.666667 & 0.00749 & 2.939 & 2.125 \\
\hline NP_001177910 & 0.66667 & 5 & 7.5 & 0.00797 & 2.907 & 2.099 \\
\hline NP_001177910 & 0.66667 & 5 & 7.5 & 0.00797 & 2.907 & 2.099 \\
\hline NP_056991 & 1 & 7.333333 & 7.333333 & 0.00199 & 2.874 & 2.701 \\
\hline XP_016881019 & 1 & 7.333333 & 7.333333 & 0.00897 & 2.874 & 2.047 \\
\hline XP_016881019 & 1 & 7.333333 & 7.333333 & 0.00897 & 2.874 & 2.047 \\
\hline XP_006716370 & 1 & 7.333333 & 7.333333 & 0.0191 & 2.874 & 1.719 \\
\hline NP_000173 & 2.33333 & 17 & 7.285714 & 0.00446 & 2.865 & 2.351 \\
\hline NP_001230209 & 2.33333 & 17 & 7.285714 & 0.00579 & 2.865 & 2.238 \\
\hline NP_001089 & 1 & 7 & 7 & 0.00048 & 2.807 & 3.315 \\
\hline NP_000681 & 1 & 7 & 7 & 0.00388 & 2.807 & 2.411 \\
\hline NP_003356 & 1 & 7 & 7 & 0.01324 & 2.807 & 1.878 \\
\hline NP_004386 & 1 & 7 & 7 & 0.03032 & 2.807 & 1.518 \\
\hline
\end{tabular}


bioRxiv preprint doi: https://doi.org/10.1101/2021.01.04.425106; this version posted January 4, 2021. The copyright holder for this preprint (which was not certified by peer review) is the author/funder, who has granted bioRxiv a license to display the preprint in perpetuity. It is made available under aCC-BY-NC-ND 4.0 International license.

\begin{tabular}{|c|c|c|c|c|c|c|}
\hline NP_490647 & 3.66667 & 24.66667 & 6.727273 & 0.01092 & 2.75 & 1.962 \\
\hline NP 055635 & 1 & 6.666667 & 6.666667 & 7E-05 & 2.737 & 4.154 \\
\hline NP_003357 & 1 & 6.666667 & 6.666667 & 0.00105 & 2.737 & 2.979 \\
\hline NP_005551 & 1 & 6.666667 & 6.666667 & 0.00302 & 2.737 & 2.521 \\
\hline NP_005994 & 1 & 6.666667 & 6.666667 & 0.00921 & 2.737 & 2.036 \\
\hline NP_005995 & 1 & 6.666667 & 6.666667 & 0.03251 & 2.737 & 1.488 \\
\hline NP_005995 & 1 & 6.666667 & 6.666667 & 0.03251 & 2.737 & 1.488 \\
\hline NP 066925 & 2.66667 & 17.66667 & 6.625 & 0.0004 & 2.728 & 3.398 \\
\hline NP_490647 & 3.66667 & 24 & 6.545455 & 0.01403 & 2.71 & 1.853 \\
\hline NP_002831 & 3.33333 & 21.66667 & 6.5 & 4.1E-05 & 2.7 & 4.385 \\
\hline NP_002380 & 0.66667 & 4.333333 & 6.5 & 0.00793 & 2.7 & 2.101 \\
\hline NP_076997 & 0.66667 & 4.333333 & 6.5 & 0.02947 & 2.7 & 1.531 \\
\hline NP_004433 & 1 & 6.333333 & 6.333333 & $8.9 \mathrm{E}-05$ & 2.663 & 4.05 \\
\hline XP_016873572 & 1 & 6.333333 & 6.333333 & 0.00132 & 2.663 & 2.878 \\
\hline NP_002834 & 1 & 6.333333 & 6.333333 & 0.00132 & 2.663 & 2.878 \\
\hline NP_005594 & 1 & 6.333333 & 6.333333 & 0.00377 & 2.663 & 2.423 \\
\hline NP_001922 & 1 & 6.333333 & 6.333333 & 0.01135 & 2.663 & 1.945 \\
\hline NP_002283 & 1 & 6.333333 & 6.333333 & 0.01613 & 2.663 & 1.792 \\
\hline NP_038470 & 1.33333 & 8.333333 & 6.25 & 0.00702 & 2.644 & 2.154 \\
\hline NP_038470 & 1.33333 & 8.333333 & 6.25 & 0.00702 & 2.644 & 2.154 \\
\hline NP_057494 & 0.66667 & 4 & 6 & 0.00056 & 2.585 & 3.25 \\
\hline XP_006713173 & 0.66667 & 4 & 6 & 0.00749 & 2.585 & 2.125 \\
\hline XP_005273672 & 1 & 6 & 6 & 0.01235 & 2.585 & 1.908 \\
\hline NP_001139433 & 4.66667 & 27 & 5.785714 & $2.9 \mathrm{E}-05$ & 2.532 & 4.533 \\
\hline NP_001291395 & 1 & 5.666667 & 5.666667 & 0.00015 & 2.503 & 3.821 \\
\hline NP_003349 & 1 & 5.666667 & 5.666667 & 0.00015 & 2.503 & 3.821 \\
\hline NP_001157666 & 1 & 5.666667 & 5.666667 & 0.00219 & 2.503 & 2.659 \\
\hline NP_006280 & 1 & 5.666667 & 5.666667 & 0.00612 & 2.503 & 2.213 \\
\hline NP_006280 & 1 & 5.666667 & 5.666667 & 0.00612 & 2.503 & 2.213 \\
\hline NP_001317939 & 1 & 5.666667 & 5.666667 & 0.04055 & 2.503 & 1.392 \\
\hline NP_001317939 & 1 & 5.666667 & 5.666667 & 0.04055 & 2.503 & 1.392 \\
\hline NP_001677 & 11 & 62 & 5.636364 & $2.2 \mathrm{E}-05$ & 2.495 & 4.66 \\
\hline NP_004068 & 2 & 11 & 5.5 & 0.00529 & 2.459 & 2.277 \\
\hline NP_000777 & 0.66667 & 3.666667 & 5.5 & 0.0158 & 2.459 & 1.801 \\
\hline NP_002380 & 0.66667 & 3.666667 & 5.5 & 0.0158 & 2.459 & 1.801 \\
\hline NP_001164187 & 0.66667 & 3.666667 & 5.5 & 0.0158 & 2.459 & 1.801 \\
\hline NP_001164187 & 0.66667 & 3.666667 & 5.5 & 0.0158 & 2.459 & 1.801 \\
\hline XP_016869235 & 1.66667 & 9 & 5.4 & 0.01955 & 2.433 & 1.709 \\
\hline NP_005836 & 1 & 5.333333 & 5.333333 & 0.0002 & 2.415 & 3.695 \\
\hline NP_005836 & 1 & 5.333333 & 5.333333 & 0.0002 & 2.415 & 3.695 \\
\hline NP_001185470 & 1 & 5.333333 & 5.333333 & 0.0002 & 2.415 & 3.695 \\
\hline NP_001309896 & 1 & 5.333333 & 5.333333 & 0.00289 & 2.415 & 2.539 \\
\hline NP_001001973 & 1 & 5.333333 & 5.333333 & 0.00289 & 2.415 & 2.539 \\
\hline
\end{tabular}


bioRxiv preprint doi: https://doi.org/10.1101/2021.01.04.425106; this version posted January 4, 2021. The copyright holder for this preprint (which was not certified by peer review) is the author/funder, who has granted bioRxiv a license to display the preprint in perpetuity. It is made available under aCC-BY-NC-ND 4.0 International license.

\begin{tabular}{|c|c|c|c|c|c|c|}
\hline NP_002296 & 1 & 5.333333 & 5.333333 & 0.00289 & 2.415 & 2.539 \\
\hline NP 714916 & 1 & 5.333333 & 5.333333 & 0.00797 & 2.415 & 2.099 \\
\hline NP_714916 & 1 & 5.333333 & 5.333333 & 0.00797 & 2.415 & 2.099 \\
\hline NP_004544 & 1 & 5.333333 & 5.333333 & 0.00797 & 2.415 & 2.099 \\
\hline NP_000117 & 1 & 5.333333 & 5.333333 & 0.00797 & 2.415 & 2.099 \\
\hline NP_006467 & 1 & 5.333333 & 5.333333 & 0.00797 & 2.415 & 2.099 \\
\hline NP_055755 & 1 & 5.333333 & 5.333333 & 0.02265 & 2.415 & 1.645 \\
\hline NP 036450 & 1 & 5.333333 & 5.333333 & 0.04064 & 2.415 & 1.391 \\
\hline NP_006787 & 1 & 5.333333 & 5.333333 & 0.04064 & 2.415 & 1.391 \\
\hline NP_006787 & 1 & 5.333333 & 5.333333 & 0.04064 & 2.415 & 1.391 \\
\hline NP_001098707 & 1.66667 & 8.666667 & 5.2 & 0.00495 & 2.379 & 2.305 \\
\hline NP_001098707 & 1.66667 & 8.666667 & 5.2 & 0.00495 & 2.379 & 2.305 \\
\hline NP_036313 & 1.33333 & 6.666667 & 5 & 0.0085 & 2.322 & 2.071 \\
\hline NP_001317170 & 0.66667 & 3.333333 & 5 & 0.02322 & 2.322 & 1.634 \\
\hline NP_001616 & 0.66667 & 3.333333 & 5 & 0.04742 & 2.322 & 1.324 \\
\hline NP_001668 & 2 & 10 & 5 & 0.00228 & 2.322 & 2.642 \\
\hline NP_056167 & 1 & 5 & 5 & 0.00228 & 2.322 & 2.642 \\
\hline NP_001014446 & 1 & 5 & 5 & 0.00228 & 2.322 & 2.642 \\
\hline NP_001166925 & 1 & 5 & 5 & 0.01613 & 2.322 & 1.792 \\
\hline NP_001166925 & 1 & 5 & 5 & 0.01613 & 2.322 & 1.792 \\
\hline NP_009034 & 1 & 5 & 5 & 0.01613 & 2.322 & 1.792 \\
\hline NP_057218 & 1 & 5 & 5 & 0.01613 & 2.322 & 1.792 \\
\hline NP_071358 & 2.33333 & 11.33333 & 4.857143 & 0.00882 & 2.28 & 2.054 \\
\hline NP_001139433 & 5.66667 & 27.33333 & 4.823529 & $9.5 \mathrm{E}-05$ & 2.27 & 4.024 \\
\hline NP_006507 & 3.66667 & 17.33333 & 4.727273 & 0.00039 & 2.241 & 3.404 \\
\hline NP_000137 & 14.3333 & 67.66667 & 4.72093 & 0.04029 & 2.239 & 1.395 \\
\hline NP_000144 & 4.33333 & 20.33333 & 4.692308 & 0.00304 & 2.23 & 2.518 \\
\hline NP_003181 & 1 & 4.666667 & 4.666667 & 0.00039 & 2.222 & 3.411 \\
\hline NP_056437 & 1 & 4.666667 & 4.666667 & 0.00039 & 2.222 & 3.411 \\
\hline NP_002309 & 1 & 4.666667 & 4.666667 & 0.00039 & 2.222 & 3.411 \\
\hline NP_689632 & 1 & 4.666667 & 4.666667 & 0.00039 & 2.222 & 3.411 \\
\hline NP_689632 & 1 & 4.666667 & 4.666667 & 0.00039 & 2.222 & 3.411 \\
\hline NP_006347 & 1 & 4.666667 & 4.666667 & 0.038 & 2.222 & 1.42 \\
\hline NP_006347 & 1 & 4.666667 & 4.666667 & 0.038 & 2.222 & 1.42 \\
\hline NP_004125 & 14 & 64.66667 & 4.619048 & 0.00061 & 2.208 & 3.215 \\
\hline NP_596867 & 18 & 82 & 4.555556 & 1E-05 & 2.188 & 4.996 \\
\hline NP_001291217 & 0.66667 & 3 & 4.5 & 0.00219 & 2.17 & 2.659 \\
\hline NP_057086 & 0.66667 & 3 & 4.5 & 0.00219 & 2.17 & 2.659 \\
\hline NP_003835 & 0.66667 & 3 & 4.5 & 0.00219 & 2.17 & 2.659 \\
\hline NP_001309997 & 0.66667 & 3 & 4.5 & 0.0249 & 2.17 & 1.604 \\
\hline XP_011511426 & 1.66667 & 7.333333 & 4.4 & 0.01908 & 2.138 & 1.719 \\
\hline XP_011511426 & 1.66667 & 7.333333 & 4.4 & 0.01908 & 2.138 & 1.719 \\
\hline NP_000427 & 1 & 4.333333 & 4.333333 & 0.00056 & 2.115 & 3.25 \\
\hline
\end{tabular}


bioRxiv preprint doi: https://doi.org/10.1101/2021.01.04.425106; this version posted January 4, 2021. The copyright holder for this preprint (which was not certified by peer review) is the author/funder, who has granted bioRxiv a license to display the preprint in perpetuity. It is made available under aCC-BY-NC-ND 4.0 International license.

\begin{tabular}{|c|c|c|c|c|c|c|}
\hline NP_000427 & 1 & 4.333333 & 4.333333 & 0.00056 & 2.115 & 3.25 \\
\hline XP_016871724 & 1 & 4.333333 & 4.333333 & 0.00056 & 2.115 & 3.25 \\
\hline XP_016871716 & 1 & 4.333333 & 4.333333 & 0.00056 & 2.115 & 3.25 \\
\hline NP_004246 & 1 & 4.333333 & 4.333333 & 0.00056 & 2.115 & 3.25 \\
\hline NP_056417 & 1 & 4.333333 & 4.333333 & 0.00749 & 2.115 & 2.125 \\
\hline NP_003650 & 1 & 4.333333 & 4.333333 & 0.00749 & 2.115 & 2.125 \\
\hline NP_006342 & 1 & 4.333333 & 4.333333 & 0.00749 & 2.115 & 2.125 \\
\hline NP 056138 & 1 & 4.333333 & 4.333333 & 0.01944 & 2.115 & 1.711 \\
\hline NP_065724 & 2.33333 & 10 & 4.285714 & 0.00802 & 2.1 & 2.096 \\
\hline NP_002071 & 8 & 34 & 4.25 & 0.00105 & 2.087 & 2.981 \\
\hline NP_002071 & 8 & 34 & 4.25 & 0.00105 & 2.087 & 2.981 \\
\hline NP_003312 & 8.33333 & 34.66667 & 4.16 & 0.00015 & 2.057 & 3.817 \\
\hline NP_003312 & 8.33333 & 34.66667 & 4.16 & 0.00015 & 2.057 & 3.817 \\
\hline NP_001070952 & 1.66667 & 6.666667 & 4 & 0.00607 & 2 & 2.217 \\
\hline NP_001001563 & 1 & 4 & 4 & 0.00653 & 2 & 2.185 \\
\hline NP_001254792 & 1 & 4 & 4 & 0.00653 & 2 & 2.185 \\
\hline XP_006715894 & 1 & 4 & 4 & 0.00653 & 2 & 2.185 \\
\hline XP_006715894 & 1 & 4 & 4 & 0.00653 & 2 & 2.185 \\
\hline NP_001317504 & 1 & 4 & 4 & 0.00653 & 2 & 2.185 \\
\hline NP_002990 & 1 & 4 & 4 & 0.00653 & 2 & 2.185 \\
\hline NP_001271342 & 1 & 4 & 4 & 0.00653 & 2 & 2.185 \\
\hline NP_006741 & 1 & 4 & 4 & 0.00653 & 2 & 2.185 \\
\hline NP_006741 & 1 & 4 & 4 & 0.00653 & 2 & 2.185 \\
\hline NP_002817 & 1 & 4 & 4 & 0.00653 & 2 & 2.185 \\
\hline NP_963906 & 1 & 4 & 4 & 0.03994 & 2 & 1.399 \\
\hline NP_004039 & 2.33333 & 9.333333 & 4 & 0.0485 & 2 & 1.314 \\
\hline NP_001327 & 22.3333 & 88.66667 & 3.970149 & 0.00215 & 1.989 & 2.667 \\
\hline NP_001193769 & 13.3333 & 52.66667 & 3.95 & 0.00219 & 1.982 & 2.66 \\
\hline NP_001080 & 10.3333 & 40.66667 & 3.935484 & 0.00026 & 1.977 & 3.581 \\
\hline XP_011532413 & 3 & 11.66667 & 3.888889 & 0.0002 & 1.959 & 3.695 \\
\hline NP_001193769 & 13.3333 & 51.66667 & 3.875 & 0.00241 & 1.954 & 2.618 \\
\hline NP_001104547 & 5 & 19.33333 & 3.866667 & 0.00579 & 1.951 & 2.237 \\
\hline NP_005262 & 4 & 15.33333 & 3.833333 & 0.00921 & 1.939 & 2.036 \\
\hline NP_001269555 & 6.66667 & 24.66667 & 3.7 & 0.00045 & 1.888 & 3.347 \\
\hline NP_001269555 & 6.66667 & 24.66667 & 3.7 & 0.00045 & 1.888 & 3.347 \\
\hline NP_004578 & 9.33333 & 34.33333 & 3.678571 & 0.00202 & 1.879 & 2.694 \\
\hline NP_004578 & 9.33333 & 34.33333 & 3.678571 & 0.00202 & 1.879 & 2.694 \\
\hline NP_001159434 & 1 & 3.666667 & 3.666667 & 0.00132 & 1.874 & 2.878 \\
\hline XP_011536954 & 1 & 3.666667 & 3.666667 & 0.00132 & 1.874 & 2.878 \\
\hline NP_001139630 & 1 & 3.666667 & 3.666667 & 0.00132 & 1.874 & 2.878 \\
\hline NP_733746 & 2 & 7.333333 & 3.666667 & 0.00718 & 1.874 & 2.144 \\
\hline NP_066972 & 1 & 3.666667 & 3.666667 & 0.01613 & 1.874 & 1.792 \\
\hline NP_ 036335 & 1 & 3.666667 & 3.666667 & 0.03902 & 1.874 & 1.409 \\
\hline
\end{tabular}


bioRxiv preprint doi: https://doi.org/10.1101/2021.01.04.425106; this version posted January 4, 2021. The copyright holder for this preprint (which was not certified by peer review) is the author/funder, who has granted bioRxiv a license to display the preprint in perpetuity. It is made available under aCC-BY-NC-ND 4.0 International license.

\begin{tabular}{|c|c|c|c|c|c|c|}
\hline NP_036335 & 1 & 3.666667 & 3.666667 & 0.03902 & 1.874 & 1.409 \\
\hline NP_001273300 & 1 & 3.666667 & 3.666667 & 0.03902 & 1.874 & 1.409 \\
\hline NP_001273300 & 1 & 3.666667 & 3.666667 & 0.03902 & 1.874 & 1.409 \\
\hline NP_002547 & 8.66667 & 31.33333 & 3.615385 & 0.00081 & 1.854 & 3.09 \\
\hline NP_005909 & 10 & 35 & 3.5 & 0.0009 & 1.807 & 3.044 \\
\hline NP_001106818 & 12 & 41.66667 & 3.472222 & 9.5E-06 & 1.796 & 5.023 \\
\hline$X P \_011541265$ & 4.33333 & 15 & 3.461538 & 0.00306 & 1.791 & 2.514 \\
\hline XP 011541265 & 4.33333 & 15 & 3.461538 & 0.00306 & 1.791 & 2.514 \\
\hline NP_008879 & 3.33333 & 11.33333 & 3.4 & 0.00194 & 1.766 & 2.713 \\
\hline NP_001094096 & 3.66667 & 12.33333 & 3.363636 & 0.00078 & 1.75 & 3.109 \\
\hline NP_001015 & 1 & 3.333333 & 3.333333 & 0.00219 & 1.737 & 2.659 \\
\hline NP_002812 & 1 & 3.333333 & 3.333333 & 0.00219 & 1.737 & 2.659 \\
\hline NP_000194 & 1 & 3.333333 & 3.333333 & 0.00219 & 1.737 & 2.659 \\
\hline NP_057055 & 1 & 3.333333 & 3.333333 & 0.00219 & 1.737 & 2.659 \\
\hline NP_001273432 & 2 & 6.666667 & 3.333333 & 0.01145 & 1.737 & 1.941 \\
\hline NP_001273432 & 2 & 6.666667 & 3.333333 & 0.01145 & 1.737 & 1.941 \\
\hline NP_002083 & 1 & 3.333333 & 3.333333 & 0.0249 & 1.737 & 1.604 \\
\hline NP_002793 & 1 & 3.333333 & 3.333333 & 0.0249 & 1.737 & 1.604 \\
\hline NP_002793 & 1 & 3.333333 & 3.333333 & 0.0249 & 1.737 & 1.604 \\
\hline NP_001924 & 1 & 3.333333 & 3.333333 & 0.0249 & 1.737 & 1.604 \\
\hline NP_037460 & 1 & 3.333333 & 3.333333 & 0.0249 & 1.737 & 1.604 \\
\hline NP_037460 & 1 & 3.333333 & 3.333333 & 0.0249 & 1.737 & 1.604 \\
\hline NP_002799 & 4 & 13 & 3.25 & 0.00146 & 1.7 & 2.835 \\
\hline NP_002799 & 4 & 13 & 3.25 & 0.00146 & 1.7 & 2.835 \\
\hline NP_060154 & 3.33333 & 10.33333 & 3.1 & 0.00934 & 1.632 & 2.03 \\
\hline NP_060154 & 3.33333 & 10.33333 & 3.1 & 0.00934 & 1.632 & 2.03 \\
\hline NP_001120699 & 7 & 21.66667 & 3.095238 & 0.00365 & 1.63 & 2.438 \\
\hline NP_056083 & 12 & 36.33333 & 3.027778 & 0.00374 & 1.598 & 2.427 \\
\hline NP_001018121 & 3.33333 & 10 & 3 & 0.01301 & 1.585 & 1.886 \\
\hline NP_001280202 & 0.66667 & 2 & 3 & 0.01613 & 1.585 & 1.792 \\
\hline NP_001280202 & 0.66667 & 2 & 3 & 0.01613 & 1.585 & 1.792 \\
\hline NP_000981 & 0.66667 & 2 & 3 & 0.01613 & 1.585 & 1.792 \\
\hline XP_016872987 & 0.66667 & 2 & 3 & 0.01613 & 1.585 & 1.792 \\
\hline NP_001139541 & 0.66667 & 2 & 3 & 0.01613 & 1.585 & 1.792 \\
\hline NP_055621 & 0.66667 & 2 & 3 & 0.01613 & 1.585 & 1.792 \\
\hline NP_001265124 & 1 & 3 & 3 & 0.02572 & 1.585 & 1.59 \\
\hline NP_001138471 & 1 & 3 & 3 & 0.02572 & 1.585 & 1.59 \\
\hline NP_001265392 & 1 & 3 & 3 & 0.02572 & 1.585 & 1.59 \\
\hline NP_001265392 & 1 & 3 & 3 & 0.02572 & 1.585 & 1.59 \\
\hline NP_149100 & 1 & 3 & 3 & 0.02572 & 1.585 & 1.59 \\
\hline NP_001352 & 1 & 3 & 3 & 0.02572 & 1.585 & 1.59 \\
\hline NP_001121132 & 1 & 3 & 3 & 0.02572 & 1.585 & 1.59 \\
\hline NP_001143 & 6 & 17.66667 & 2.944444 & 0.00038 & 1.558 & 3.421 \\
\hline
\end{tabular}


bioRxiv preprint doi: https://doi.org/10.1101/2021.01.04.425106; this version posted January 4, 2021. The copyright holder for this preprint (which was not certified by peer review) is the author/funder, who has granted bioRxiv a license to display the preprint in perpetuity. It is made available under aCC-BY-NC-ND 4.0 International license.

\begin{tabular}{|c|c|c|c|c|c|c|}
\hline NP_076424 & 3 & 8.666667 & 2.888889 & 0.03251 & 1.531 & 1.488 \\
\hline NP 003642 & 5.66667 & 16.33333 & 2.882353 & 0.04742 & 1.527 & 1.324 \\
\hline NP_001192183 & 2.33333 & 6.666667 & 2.857143 & 0.00436 & 1.515 & 2.361 \\
\hline NP_009216 & 2 & 5.666667 & 2.833333 & 0.02539 & 1.503 & 1.595 \\
\hline NP 877420 & 3.33333 & 9.333333 & 2.8 & 0.00559 & 1.485 & 2.253 \\
\hline NP_001171903 & 1.66667 & 4.666667 & 2.8 & 0.03347 & 1.485 & 1.475 \\
\hline NP_001143 & 7.66667 & 21.33333 & 2.782609 & 0.00039 & 1.476 & 3.404 \\
\hline NP 054735 & 13.6667 & 38 & 2.780488 & 5.3E-05 & 1.475 & 4.276 \\
\hline NP_054735 & 13.6667 & 38 & 2.780488 & $5.3 \mathrm{E}-05$ & 1.475 & 4.276 \\
\hline NP_001627 & 6 & 16.66667 & 2.777778 & 0.00054 & 1.474 & 3.27 \\
\hline NP_001367 & 3 & 8.333333 & 2.777778 & 0.00718 & 1.474 & 2.144 \\
\hline NP_955472 & 53.3333 & 147.3333 & 2.7625 & 0.00247 & 1.466 & 2.607 \\
\hline NP_001189414 & 12 & 33 & 2.75 & 0.00301 & 1.459 & 2.522 \\
\hline NP_113651 & 1.33333 & 3.666667 & 2.75 & 0.03517 & 1.459 & 1.454 \\
\hline NP_003944 & 1.33333 & 3.666667 & 2.75 & 0.03517 & 1.459 & 1.454 \\
\hline NP_001825 & 2 & 5.333333 & 2.666667 & 0.00056 & 1.415 & 3.25 \\
\hline NP_689601 & 1 & 2.666667 & 2.666667 & 0.00749 & 1.415 & 2.125 \\
\hline NP_689450 & 1 & 2.666667 & 2.666667 & 0.00749 & 1.415 & 2.125 \\
\hline NP_001316143 & 1 & 2.666667 & 2.666667 & 0.00749 & 1.415 & 2.125 \\
\hline NP_057292 & 1 & 2.666667 & 2.666667 & 0.00749 & 1.415 & 2.125 \\
\hline NP_001000 & 1 & 2.666667 & 2.666667 & 0.00749 & 1.415 & 2.125 \\
\hline NP_079172 & 1 & 2.666667 & 2.666667 & 0.00749 & 1.415 & 2.125 \\
\hline NP_001245366 & 1 & 2.666667 & 2.666667 & 0.00749 & 1.415 & 2.125 \\
\hline NP_006779 & 3.66667 & 9.666667 & 2.636364 & 0.00129 & 1.399 & 2.888 \\
\hline NP_003036 & 6.33333 & 16.66667 & 2.631579 & 2.6E-05 & 1.396 & 4.591 \\
\hline NP_000099 & 4.33333 & 11.33333 & 2.615385 & 0.00012 & 1.387 & 3.922 \\
\hline NP_789782 & 5 & 13 & 2.6 & 0.04179 & 1.379 & 1.379 \\
\hline NP_001351 & 1.66667 & 4.333333 & 2.6 & 0.04742 & 1.379 & 1.324 \\
\hline NP_710154 & 3 & 7.666667 & 2.555556 & 0.0249 & 1.354 & 1.604 \\
\hline NP_002818 & 3 & 7.666667 & 2.555556 & 0.04881 & 1.354 & 1.311 \\
\hline NP_631918 & 9 & 22.66667 & 2.518519 & 0.00424 & 1.333 & 2.373 \\
\hline NP_001138303 & 5.33333 & 13.33333 & 2.5 & 0.00582 & 1.322 & 2.235 \\
\hline NP_003477 & 3.33333 & 8.333333 & 2.5 & 0.022 & 1.322 & 1.658 \\
\hline NP_006422 & 13.6667 & 34 & 2.487805 & 0.02036 & 1.315 & 1.691 \\
\hline NP_002625 & 7.66667 & 19 & 2.478261 & 0.00105 & 1.309 & 2.979 \\
\hline NP_002625 & 7.66667 & 19 & 2.478261 & 0.00105 & 1.309 & 2.979 \\
\hline XP_016873370 & 7 & 17.33333 & 2.47619 & 0.00207 & 1.308 & 2.685 \\
\hline XP_016873370 & 7 & 17.33333 & 2.47619 & 0.00207 & 1.308 & 2.685 \\
\hline NP_848927 & 3.66667 & 9 & 2.454545 & 0.00377 & 1.295 & 2.423 \\
\hline NP_003833 & 2.33333 & 5.666667 & 2.428571 & 0.02411 & 1.28 & 1.618 \\
\hline NP_001315615 & 25 & 60.66667 & 2.426667 & 0.00238 & 1.279 & 2.624 \\
\hline XP_011534504 & 5 & 12 & 2.4 & 0.00374 & 1.263 & 2.427 \\
\hline NP_002148 & 18.6667 & 44.66667 & 2.392857 & 0.00296 & 1.259 & 2.528 \\
\hline
\end{tabular}


bioRxiv preprint doi: https://doi org/10.1101/2021.01.04.425106; this version posted January 4, 2021. The copyright holder for this preprint (which was not certified by peer review) is the author/funder, who has granted bioRxiv a license to display the preprint in perpetuity. It is made available under aCC-BY-NC-ND 4.0 International license.

\begin{tabular}{|c|c|c|c|c|c|c|}
\hline NP_000375 & 5.33333 & 12.66667 & 2.375 & 0.00268 & 1.248 & 2.572 \\
\hline NP_002435 & 5.33333 & 12.66667 & 2.375 & 0.04911 & 1.248 & 1.309 \\
\hline NP_001656 & 3.66667 & 8.666667 & 2.363636 & 0.00045 & 1.241 & 3.349 \\
\hline$N P \_006030$ & 3.66667 & 8.666667 & 2.363636 & 0.00257 & 1.241 & 2.59 \\
\hline XP_005259654 & 1 & 2.333333 & 2.333333 & 0.01613 & 1.222 & 1.792 \\
\hline XP_005259654 & 1 & 2.333333 & 2.333333 & 0.01613 & 1.222 & 1.792 \\
\hline NP_001018080 & 1 & 2.333333 & 2.333333 & 0.01613 & 1.222 & 1.792 \\
\hline NP_001128567 & 1 & 2.333333 & 2.333333 & 0.01613 & 1.222 & 1.792 \\
\hline NP_001311211 & 1 & 2.333333 & 2.333333 & 0.01613 & 1.222 & 1.792 \\
\hline NP_001280134 & 1 & 2.333333 & 2.333333 & 0.01613 & 1.222 & 1.792 \\
\hline NP_000683 & 1 & 2.333333 & 2.333333 & 0.01613 & 1.222 & 1.792 \\
\hline XP_016876295 & 1 & 2.333333 & 2.333333 & 0.01613 & 1.222 & 1.792 \\
\hline XP_016876295 & 1 & 2.333333 & 2.333333 & 0.01613 & 1.222 & 1.792 \\
\hline NP_689518 & 1 & 2.333333 & 2.333333 & 0.01613 & 1.222 & 1.792 \\
\hline NP_919415 & 19.6667 & 45.66667 & 2.322034 & 0.00132 & 1.215 & 2.879 \\
\hline NP_002148 & 20.3333 & 46.66667 & 2.295082 & 0.00212 & 1.199 & 2.674 \\
\hline NP_004550 & 8.66667 & 19.33333 & 2.230769 & 0.00103 & 1.158 & 2.989 \\
\hline NP_001020331 & 3.33333 & 7.333333 & 2.2 & 0.00582 & 1.138 & 2.235 \\
\hline NP_001020331 & 3.33333 & 7.333333 & 2.2 & 0.00582 & 1.138 & 2.235 \\
\hline$X P \_011529429$ & 7.66667 & 16.66667 & 2.173913 & 0.0226 & 1.12 & 1.646 \\
\hline NP_001072989 & 15.6667 & 33.66667 & 2.148936 & 0.04361 & 1.104 & 1.36 \\
\hline$N P \_004550$ & 9.33333 & 20 & 2.142857 & 0.00306 & 1.1 & 2.514 \\
\hline NP_001002814 & 5.66667 & 12 & 2.117647 & 0.00386 & 1.082 & 2.413 \\
\hline$X P \_005258336$ & 46.6667 & 98.33333 & 2.107143 & 0.00015 & 1.075 & 3.825 \\
\hline NP_002061 & 22.6667 & 47.66667 & 2.102941 & 0.0005 & 1.072 & 3.3 \\
\hline NP_001336864 & 4.66667 & 9.666667 & 2.071429 & 0.02846 & 1.051 & 1.546 \\
\hline NP_002363 & 5.33333 & 11 & 2.0625 & 0.02227 & 1.044 & 1.652 \\
\hline NP_002363 & 5.33333 & 11 & 2.0625 & 0.02227 & 1.044 & 1.652 \\
\hline NP_938148 & 34 & 70 & 2.058824 & 0.00051 & 1.042 & 3.292 \\
\hline NP_000503 & 10.3333 & 21 & 2.032258 & 0.03594 & 1.023 & 1.444 \\
\hline NP_000503 & 10.3333 & 21 & 2.032258 & 0.03594 & 1.023 & 1.444 \\
\hline NP_002385 & 23 & 46.33333 & 2.014493 & 4E-06 & 1.01 & 5.4 \\
\hline NP_002385 & 23 & 46.33333 & 2.014493 & 4E-06 & 1.01 & 5.4 \\
\hline NP_061849 & 3.33333 & 6.666667 & 2 & 0.00211 & 1 & 2.676 \\
\hline NP_055422 & 2.33333 & 4.666667 & 2 & 0.00776 & 1 & 2.11 \\
\hline$X P \_016860258$ & 3.66667 & 7.333333 & 2 & 0.00793 & 1 & 2.101 \\
\hline NP_004595 & 2.66667 & 5.333333 & 2 & 0.02322 & 1 & 1.634 \\
\hline NP_004595 & 2.66667 & 5.333333 & 2 & 0.02322 & 1 & 1.634 \\
\hline
\end{tabular}




\section{Supplementary Table 2: Cohort of $>2$ fold significantly enriched PDA lysosomal proteins associated with vesicle trafficking and endocytosis}

Accession

Number

NP_005836

NP_001690

NP_056437

XP_011512751

NP_001120699

NP_056083

NP_009216

NP_055755

NP_000262 / XP

NP_001002814

NP_065724

NP_003181

NP_919415

NP_057292

NP_001093

NP_004915

NP_001315615

NP_001020527

NP_000375

XP_006718556

NP_004039

NP_001327

NP_789782

NP_057494

NP_001825

NP_001098707

NP_001265392

NP_001367
Protein Name

ATP binding cassette subfamily $C$ member $4(A B C C 4)$

$A X L$ receptor tyrosine kinase $(A X L)$

CCZ1 homolog, vacuolar protein trafficking and

biogenesis associated(CCZ1)

CD2 associated protein(CD2AP)

CD59 molecule(CD59)

DnaJ heat shock protein family (Hsp40) member

C13(DNAJC13)

GABA type $A$ receptor associated protein like

2(GABARAPL2)

MON1 homolog $B$, secretory trafficking

associated(MON1B)

_NPC intracellular cholesterol transporter 1(NPC1)

$x$

RAB11 family interacting protein 1(RAB11FIP1)

RAB22A, member RAS oncogene family(RAB22A)

TAP binding protein(TAPBP)

VAMP associated protein A(VAPA)

VPS28, ESCRT-I subunit(VPS28)

actinin alpha 1 (ACTN1)

actinin alpha 4 (ACTN4)

Present

within the GO

Biological

Process

category

"endocytosis"

GO: 0006897

$\mathrm{X}$

$\mathrm{X}$

$x$

amyloid beta precursor like protein 2(APLP2)

anoctamin 6(ANO6)

apolipoprotein B(APOB)

atlastin GTPase 3(ATL3)

beta-2-microglobulin(B2M)

cathepsin Z(CTSZ)

charged multivesicular body protein 4B(CHMP4B)

charged multivesicular body protein 5(CHMP5)

clathrin light chain $B(C L T B)$

coronin $1 \mathrm{C}(\mathrm{CORO} 1 \mathrm{C})$

$x$

dynamin 1 like(DNM1L)

$x$

$x$

$x$

dynein cytoplasmic 1 heavy chain 1 (DYNC1H1) 
NP_003557 early endosome antigen 1(EEA1)

$X$

NP_005219 epidermal growth factor receptor(EGFR)

NP_001104547 ezrin(EZR)

$X P \_016871724$ family with sequence similarity 21 member A(FAM21A)

XP_011529429 filamin A(FLNA)

XP_006713173 golgin A4(GOLGA4)

NP_001273432 heat shock protein family H (Hsp110) member 1(HSPH1) X

NP_001138471 integrin subunit alpha V(ITGAV)

NP_596867 integrin subunit beta 1(ITGB1)

NP_001317939 intersectin 1(ITSN1)

NP_000518 low density lipoprotein receptor(LDLR)

NP_002309 lysyl oxidase like 2(LOXL2)

NP_006030 mannose receptor C type 2(MRC2)

$x$

$x$

$x$

NP_002435

moesin(MSN)

NP_002547

oxysterol binding protein like $1 \mathrm{~A}(\mathrm{OSBPL} 1 \mathrm{~A})$

NP_001157666 perilipin 3(PLIN3)

NP_037364

programmed cell death 6 interacting protein(PDCD6IP)

protein kinase $C$ and casein kinase substrate in neurons

NP_001171903

3(PACSIN3)

$X$

NP_002818 1(PTPN1)

NP_002817

quiescin sulfhydryl oxidase 1(QSOX1)

NP_006779

ralA binding protein 1(RALBP1)

NP_000593

serpin family $E$ member 1 (SERPINE1)

$x$

NP_001311211

signal transducing adaptor molecule(STAM)

XP_011541265

sortilin related receptor 1 (SORL1)

NP_689450

sorting nexin 11(SNX11)

XP_006717309

spectrin alpha, non-erythrocytic 1(SPTAN1)

XP_005264575

spectrin beta, non-erythrocytic 1(SPTBN1)

NP_002990

syndecan 4(SDC4)

NP_001007069 / syndecan binding protein(SDCBP)

$x$

NP_004595

syntaxin 4 (STX4)

NP_006280

talin 1(TLN1)

NP_001310245

transglutaminase 2(TGM2)

NP_006283

tumor susceptibility 101(TSG101)

$X$

NP_060154 vacuolar protein sorting 13 homolog C(VPS13C)

NP_001273300 vesicle trafficking 1(VTA1) 
Supplementary Table 3: Clinicopathological characteristics and group membership in ULCA TMA (encompassing resected stage I/II pancreatic cancer)

\begin{tabular}{|c|c|c|c|}
\hline \multirow{2}{*}{$\begin{array}{c}\text { Clinicopathologic } \\
\text { Category }\end{array}$} & \multicolumn{3}{|c|}{ MYOF IHC staining } \\
\hline & Low & High & $\begin{array}{c}p- \\
\text { value }^{a}\end{array}$ \\
\hline Total patients (n) & 105 & 31 & $\mathrm{~N} / \mathrm{A}$ \\
\hline Median Age (yrs) & 63.7 & 65.4 & $\mathrm{~N} / \mathrm{A}$ \\
\hline $\begin{array}{l}\text { Gender } \\
\text { Male } \\
\text { Female }\end{array}$ & $\begin{array}{l}63(60 \%) \\
42(40 \%)\end{array}$ & $\begin{array}{l}10(32 \%) \\
21(68 \%)\end{array}$ & 0.006 \\
\hline $\begin{array}{l}\text { Histologic grade } \\
\text { Low } \\
\text { High }\end{array}$ & $\begin{array}{l}61(58 \%) \\
44(42 \%)\end{array}$ & $\begin{array}{l}14(45 \%) \\
17(55 \%)\end{array}$ & 0.2 \\
\hline $\begin{array}{l}\text { pT (categorized) } \\
\text { T1+T2 } \\
\text { T2 } \\
\text { T3 }\end{array}$ & $\begin{array}{l}17(16 \%) \\
45(43 \%) \\
43(41 \%)\end{array}$ & $\begin{array}{c}8(26 \%) \\
9(29 \%) \\
14(45 \%)\end{array}$ & 0.29 \\
\hline $\begin{array}{l}\text { pN (categorized) } \\
\text { N0 } \\
\text { N1 }\end{array}$ & $\begin{array}{l}54(51 \%) \\
51(49 \%)\end{array}$ & $\begin{array}{l}12(39 \%) \\
19(61 \%)\end{array}$ & 0.21 \\
\hline $\begin{array}{l}\text { AJCC Stage } \\
\text { II }\end{array}$ & $\begin{array}{l}33(31 \%) \\
72(69 \%)\end{array}$ & $\begin{array}{c}6(19 \%) \\
25(81 \%)\end{array}$ & 0.19 \\
\hline $\begin{array}{l}\text { Surgical Margins } \\
\text { R0 } \\
\text { R1 }\end{array}$ & $\begin{array}{l}92(88 \%) \\
13(12 \%)\end{array}$ & $\begin{array}{c}28(90 \%) \\
3(10 \%)\end{array}$ & 0.68 \\
\hline $\begin{array}{l}\text { Tumor size (categ } \\
\quad<3 \mathrm{~cm} \\
\quad \geq 3 \mathrm{~cm}\end{array}$ & $\begin{array}{l}\text { ized) } \\
64(61 \%) \\
41(39 \%)\end{array}$ & $\begin{array}{l}16(52 \%) \\
15(48 \%)\end{array}$ & 0.35 \\
\hline
\end{tabular}

${ }^{a} p$-values for Chi square test; Low staining =bottom three quartiles (histoscore 0-5); high staining=top quartile (histoscore $>5-9$ ). Variables based on the 7 th edition of the AJCC/UICC TNM staging system for pancreatic cancer. 


\section{Supplementary Table 4 : Cox proportional hazard models for prognostic factors}

\section{A) Univariant Cox Analysis of overall survival in pancreatic cancer TMA}

\begin{tabular}{|l|c|c|}
\hline \multirow{2}{*}{ IHC Group } & $\begin{array}{c}\text { Adjusted } \\
\mathrm{HR}^{*} \\
(95 \% \mathrm{Cl})\end{array}$ & p-value $^{\dagger}$ \\
\hline $\begin{array}{l}\text { High MYOF } \\
\text { expression }\end{array}$ & $\begin{array}{c}2.03(1.29 \\
-3.19)\end{array}$ & 0.002 \\
\hline
\end{tabular}

B) Multivariant proportional hazards analysis of overall survival in pancreatic cancer TMA

\begin{tabular}{|c|c|c|}
\hline \multirow[b]{2}{*}{ Variable } & \multicolumn{2}{|c|}{ Overall Survival } \\
\hline & $\begin{array}{c}\text { Adjusted } \\
\text { HR }^{*} \\
(95 \% \mathrm{Cl})\end{array}$ & $p$-value ${ }^{\dagger}$ \\
\hline $\begin{array}{l}\text { High MYOF } \\
\text { expression }\end{array}$ & $\begin{array}{c}1.98(1.25 \\
-3.12)\end{array}$ & 0.004 \\
\hline $\begin{array}{l}\text { High grade } \\
\text { histology }\end{array}$ & $\begin{array}{c}2.04(1.34 \\
-3.09)\end{array}$ & 0.001 \\
\hline $\begin{array}{l}\text { Lymph node } \\
\text { involvement }\end{array}$ & $\begin{array}{c}2.03(1.34 \\
-3.08)\end{array}$ & 0.001 \\
\hline $\begin{array}{c}\text { Lower pT } \\
\text { status }\end{array}$ & $\begin{array}{c}0.70(0.53 \\
-0.92)\end{array}$ & 0.002 \\
\hline
\end{tabular}

"HR (Hazard Ratio): HR of 1 indicates no difference between the two groups of patients for the listed variable, while a HR $>1$ indicates an increased risk of death/failure for the group listed. $† p$-value from Chi-square test using the Cox proportional hazards model. The following covariates were initially evaluated in a Cox proportional hazards model with backward selection: 1. Lymph node involvement (pN0 vs. pN1), 2. Histologic grade (Low vs. High), 3. T-stage (pT1-pT3), 4. Tumor size ( $<3$ vs. $\geq 3 \mathrm{~cm}$ ), 5 . Age ( $<60 \mathrm{vs.} \geq 60 \mathrm{yrs}$ ), 6 . Gender, 7 . Surgical margin status (negative vs. positive), 8. MYOF expression (low vs. high). 